\title{
Simultaneous Mass Detection for Direct Inlet Mass Spectrometry
}

\author{
R. L. Gordon
}

May 1979

Prepared for the U.S. Department of Energy under Contract EY-76-C-06-1830

Pacific Northwest Laboratory Operated for the U.S. Department of Energy by Battelle Memorial Institute 


\title{
NOTICE
}

This report was prepared as an account of work sponsored by the United States Government. Neither the United States nor the Department of Energy, nor any of their employees, nor any of their contractors. subcontractors, or their employees, makes any warranty, express or implied, or assumes any legal liability or responsibility for the accuracy, completeness or usefulness of any information, apparatus, product or process disclosed, or represents that its use would not infringe privately owned rights.

The views, opinions and conclusions contained in this report are those of the contractor and do not necessarily represent those of the United States Government or the United States Department of Energy.

\author{
PACIFIC NORTHWEST LABORATORY \\ operated by \\ BATTELLE \\ for the \\ UNITED STATES DEPARTMENT OF ENERGY \\ Under Contract EY-76-C-06-1830
}
Printed in the United States of America Available from National Technical Information Service United States Department of Commerce 5285 Port Royal Road Springfield, Virginia 2215

Price: Printed Copy $\mathbf{s}$ $\because$ Microfiche $\$ 3.00$

NTIS

-Pages Selling Price

$\begin{array}{ll}001-025 & \$ 4.00 \\ 026-050 & \$ 4.50 \\ 051-075 & \$ 5.25 \\ 076-100 & \$ 6.00 \\ 101-125 & \$ 6.50 \\ 126-150 & \$ 7.25 \\ 151-175 & \$ 8.00 \\ 176-200 & \$ 9.00 \\ 201-225 & \$ 9.25 \\ 226-250 & \$ 9.50 \\ 251-275 & \$ 10.75 \\ 276-300 & \$ 11.00\end{array}$


PNL-2728

UC-37

33679000491730

SIMULTANEOUS MASS DETECTION FOR

DIRECT INLET MASS SPECTROMETRY

R. L. Gordon

May 1979

Prepared for the U.S. Department of Energy under Contract EY-76-C-06-1830

Pacific Northwest Laboratory

Richland, Washington 99352 



\section{CONTENTS}

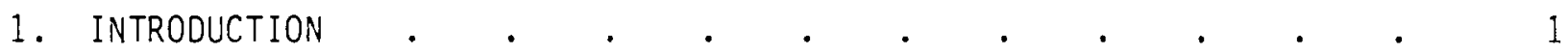

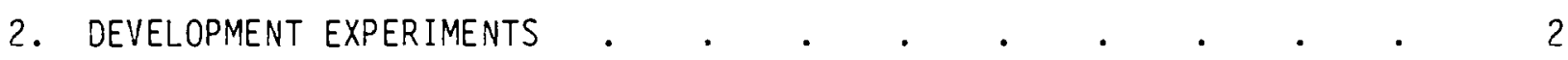

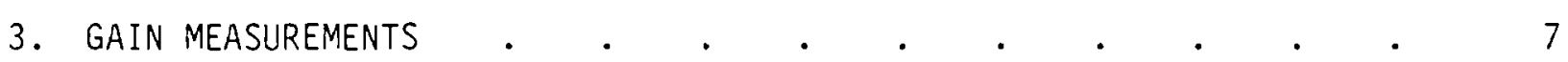

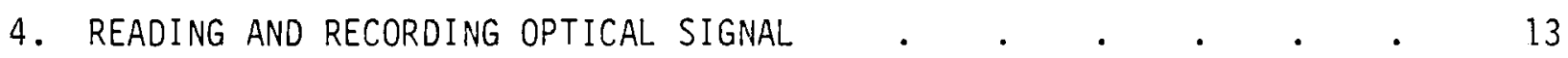

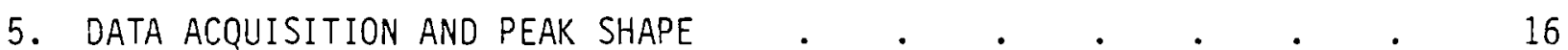

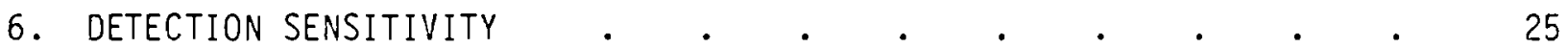

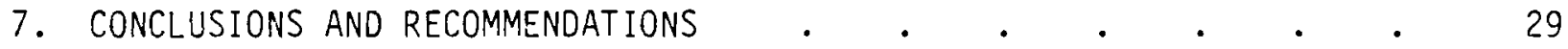

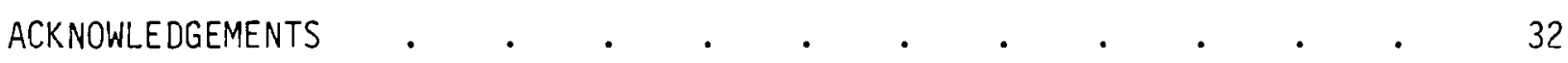

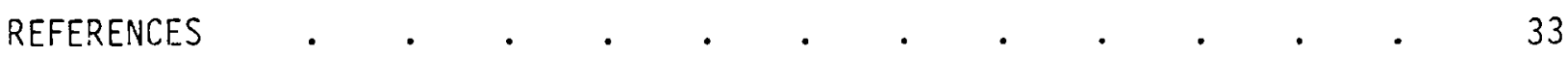

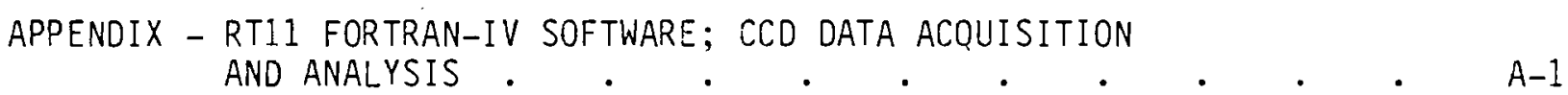




\section{FIGURES}

1 Schematic of Mass Analyzer and Vacuum System . . . . . . . 3

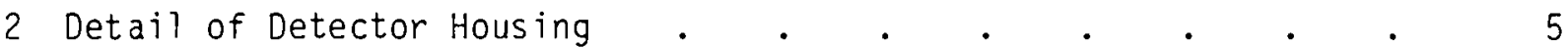

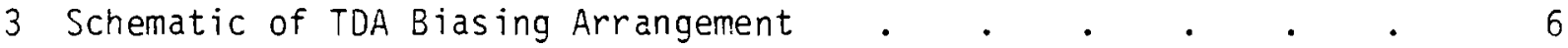

4 Photograph of Operating TDA-Phosphor Screen . . . . . . 6

5 Observed TDA Gain for 3000-eV H2 Ions for Indicated Currents . . . . . . . . . . . . . . 8

6 TDA Current Transfer Characteristics for 3000-eV $\mathrm{H}_{2}$ Ions . . 9

7 Observed TDA Gain for Krypton Isotopes at Indicated Currents . 10

8 Output Current as a Function of Position of the Incident Beam on the Face of the TDA, $\mathrm{H}_{2}$ Ions at the Indicated

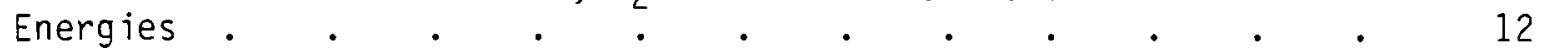

9 Comparison of Video (a) Sample-and-Hold (b) Output of CCD . . 15

10 Schematic Indicating Connections Between Charge-Coupled Device

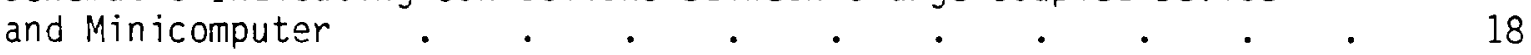

11 CCD Dark Signal at $-40^{\circ} \mathrm{C}$ and a Data Rate of $1785 \mathrm{~s}^{-1}$. . . 19

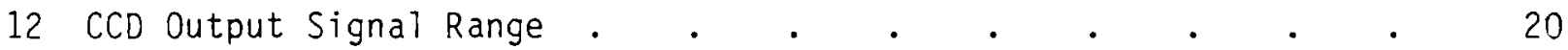

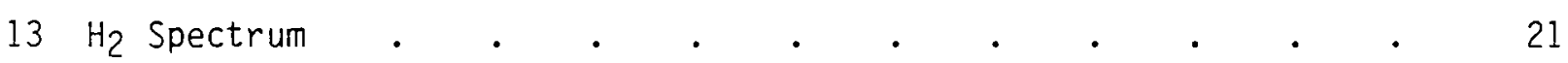

14 Central 256 Points of the Spectrum of Figure 13 . $\quad$. . . 22

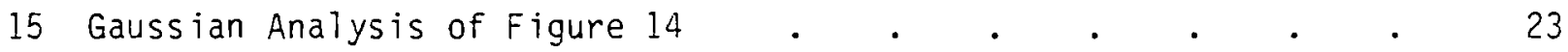

16 Krypton Spectrum: Masses $78,80,82,83,84$, and 86 . 4 . 24

17 CCO Output Versus Incident Ion Current of $\mathrm{kr}$ Isotopes . . . . 26

18 CCD Output Versus Integrated Ion Current for $\mathrm{Kr}$ Isotopes 82,
84 , and 86 


\section{SIMULTANEOUS MASS DETECTION FOR}

DIRECT INLET MASS SPECTROMETRY

\section{INTRODUCTION}

The evolution of analytical techniques for application in trace analys is has led to interest in practical methods for real-time monitoring. Direct inlet mass spectrometry (DIMS) has been the subject of considerable activity in recent years. In applications for the monitoring of particulate content of the atmosphere, a DIMS instrument consists of an inlet system designed to permit particles entrained in the inlet air stream to strike a hot, oxidized rhenium filament which serves as a surface ionization source. A mass analyzer and detection system then permits identification of the elemental composition of particulates which strike the filament.

Several approaches to DIMS instrumentation have been discussed. The or iginal work was performed by Davis using a sector instrument. ${ }^{(1)}$ Meyers and Fite, ${ }^{(2)}$ as well as Lassiter and Moen, ${ }^{(3)}$ used a quadrupole mass filter as the analyzer. Stoffels and Lagergren have developed a magnetic sector instrument for monitoring particulates in the atmosphere. ${ }^{(4)}$ These instruments are all configured for single mass detection. The quadrupole mass filter is capable of rapid scanning but still detects masses one-at-a-time. Magnetic sector instruments provide dispersion necessary for simultaneous observation of masses produced by the ionization of individual particles, but are usually arranged with slits in the detector end to improve resolution and hence are commonly thought of and used for single mass detection.

Single mass detection is not adequate for unambiguous identification of the elements contained in a single particle striking the filament. A particle striking the hot filament will dissociate, producing products, some of which will be ionized by surface ionization. Ions produced by one particle will appear at the detector essentially simultaneously. Consequently a means for simultaneous detection of ions produced by individual particulate impacts is 
required to establish a definite time correlation between ionic signals at different masses.

The Pacific Northwest Laboratory (PNL) has evaluated an approach to simultaneous mass detection for application in a DIMS analyzer. The chosen concept, first suggested by Beottger, (5) and by Giffen, Boettger, and Norris, ${ }^{(6)}$ has also been discussed by others. (7) A phosphor-backed multiplier channel plate (MCP) is positioned at the focal plane of the mass spectrometer to serve as the ion detector. This device serves as a transducer to convert the ion image of the entrance slit at each mass position on the focal plane into individual bars of light on the phosphor screen. In previous work this optical signal was recorded numerically using a vidicon camera. In our experiments, a specially designed lens systems was used to focus the light from the phosphor onto a charge-coupled-device (CCD), consisting of a linear array of photodetectors and supporting circuitry on the same single-crystal silicon chip. In eventual application a fiber optics arrangement will be used to bring the optical signal to the CCD. This method of mass detection permits simultaneous detection of all masses with sequential read-out of stored signals via the electronics of the CCD.

This paper describes work sponsored by the Department of Energy's Division of Chemical Sciences and conducted by PNL to evaluate this electrooptical detector concept. Section 2 descrites the mass spectometer assembled for these experiments. Section 3 reports results of measurements of MCP gain. The optical system and the operation of the CCD is described in Section 4, and data acquisition techniques and observations on peak shape are reported in. Section 5. Section 6 describes results of sensitivity measurements using krypton gas and Section 7 presents conclusions and recommendations, while software developed for computerized data acquisition and analys is is presented in the Appendix.

\section{DEVELOPMENT EXPERIMENTS}

A mass analyzer was assembled for the specific purpose of evaluating different approaches to simultaneous detection. The analyzer and vacuum system, shoivn schematically in Figure 1 , is constructed primarily of commercially 


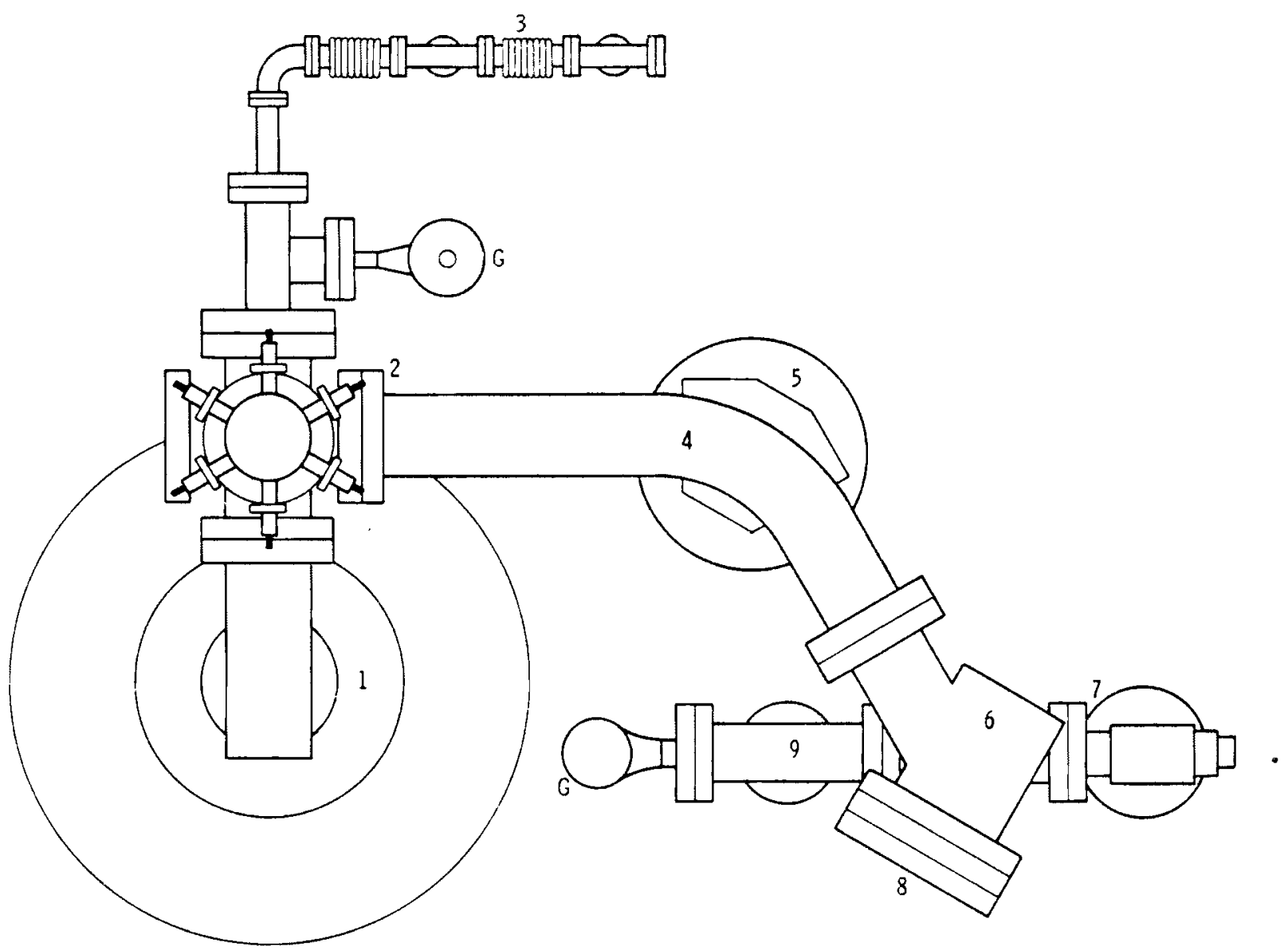

FIGURE 1. Schematic of Mass Analyzer and Vacuum System 1) Valve to Diffusion Pump and Liquid Nitrogen Trap; 2) Source Volume and Electrical Feedthroughs; 3) Gas Manifold; 4) Analyzer Orift Space; 5) Magnet; 6) Detector Housing; 7) Sorption Pump; 8) Vacuum Viewport; 9) Tubulation to Ion Pump; G) Bayard-Alpert Ionization Gauges.

available components. Only four components are of special design. These include the mass tube, two feedthrough collars for supplying potentials in the source region, and a special detector housing with appropriate feedthroughs to support electrical requirements of the MCP. The mass tube has inside dimensions of $5 \mathrm{~cm} \times 0.95 \mathrm{~cm}$ to permit transmission of several masses. All components bolt together on demountable, bakeable ultra-high-vacuum flanges for ease in changing configurations. A 800-liter/s oil diffusion pump with a liquid nitrogen trap is used at the source end of the system, while a 26-liter/s ion pump operates continuously at the detector end. Sources of reagent-grade krypton and xenon gas were provided as mass standards for the 
work. One-liter flasks of each gas mounted on independent leak valves communicate with the source region yia a simple manifold. The entire vacuum system can be baked to $400^{\circ} \mathrm{C}$ should a need arise for performing experiments in ultra-high vacuum.

Ionization is accomplished by a simple electron bombardment source consisting of two helical tungsten filaments mounted on either side of a cage of 95\%-transparent tungsten mesh positioned over the entrance aperture of the ion lens. The ion lens is a Nier-type thick lens ${ }^{(8)}$ used in other mass spectrometers in our laboratory. The instrument uses a $15-\mathrm{cm}-$ radius, $60^{\circ}$-sector electromagnet capable of producing 6,000 gauss at $510 \mathrm{~mA}$ of magnet current.

Experiments reported here were conducted with a 40-mm-diameter tandem dual array (TDA) MCP available from LSE Division of Varian Associates, (Varian No. VUW 8998). The MCP consists of a close-packed array of 12.5-um-dia tubes placed at 15-um centers. The TDA, or "chevron" device used here consists of two MCPs stacked together. An individual array has a quoted resolution of 28 line pairs per millimeter while that for the TOA is 14 line pairs per millimeter. The MCP is backed by a phosphor screen with P-20 (green) response. The combination of high efficiency of the P-20 phosphor and significant sensitivity for the green $5682-\AA$ emission possessed by the silicon photodiodes used for light detection determined the choice of the P-20 phosphor. Ions striking the face of the MCP generate electrons which undergo multiplication in the small tubes of the MCP. The electron output of the array is therefore in registry with the positions at which ions struck the front face. Electrons resulting from localized gain in the MCP are then accelerated a short distance (proximity focused) to the phosphor so the light distribution on the phosphor is essentially an image of the number and positions of ions which struck the front face of the MCP. The entrance slit images which are formed at the focal $p l a n e$ of the mass spectrometer are re-imaged by the phosphor screen.

The MCP-Phosphor assembly is mounted on a compression ring which slips into the inside diameter of the detector housing as shown in Figure 2 . The system is held firmly so that the front face of the MCP lies in the focal plane of the mass analyzer by three set screws in the compression ring. The position was established by carefully constructed stand-off rods which bottom 


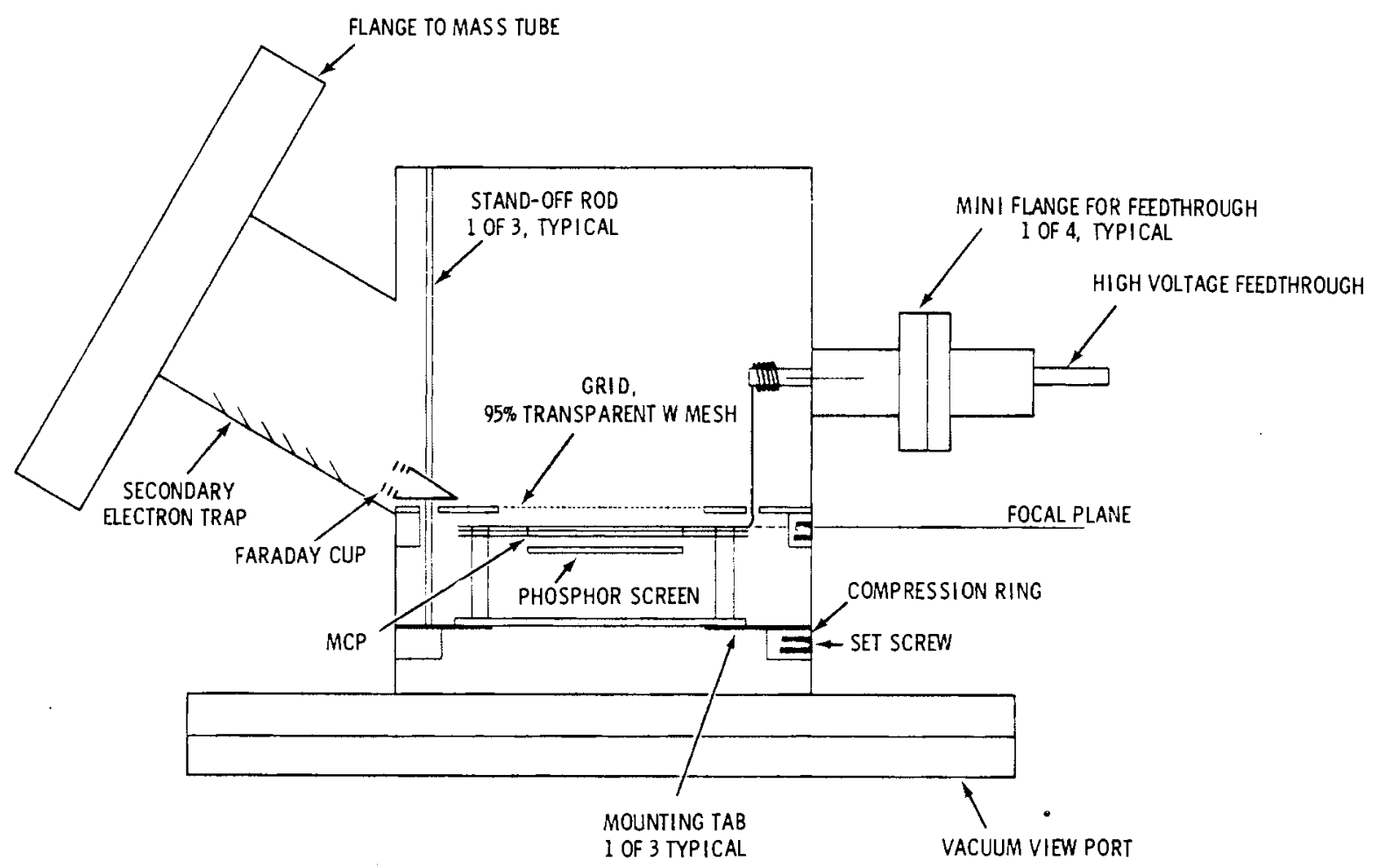

FIGURE 2. Detail of Detector Housing (The front face of the TDA-MCP lies on the local plane, as shown.)

on the back side of the detector housing. A grounded grid of 95\%-transparent tungsten mesh, mounted on its own compression ring, serves to define the ground potential for ion accelerating voltage and sits about $1 / 2 \mathrm{~mm}$ in front of the theoretical focal plane of the instrument.

The presence of the grounded grid permits two possible methods of biasing the MCP-Phosphor system. In the first method, the front face of the MCP is held at ground potential and the rear face at the MCP operating voltage, $V_{C}$. The second method puts the front face of the MCP at - $V_{c}$ with the rear face at ground potential. With the second method there exists post-acceleration of the mass-analyzed ions into the face of the MCP. This method, however, introduced considerable distortion in slit images near the edge of the MCP because of the non-uniform post-accelerating field which was due to the finite extent of the grid. Though the second biasing method produced noticeably 
brighter images on the phosphor, the distortion forced a choice of the first biasing method for subsequent work. A schematic of the MCP-Phosphor biasing arrangement is shown in Figure 3. Siightly better performance of the phosphor could be expected if the phosphor bias supply were floatable. Such a supply was not available for these experiments.

A closeup of the phosphor screen is shown in Figure 4. The photo shows the mass spectrum produced by residual gas in the mass 44 region. Six masses are visible in the photo. The brightest image is for mass 44 .
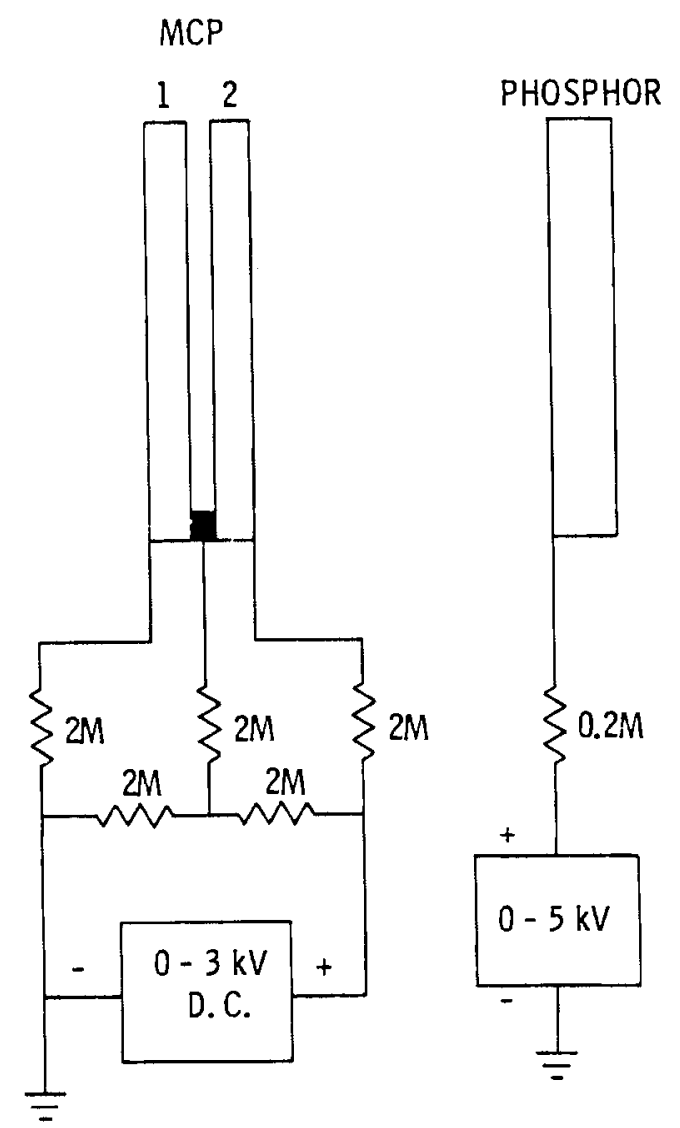

FIGURE 3. Schematic of TDA Biasing Arrangement

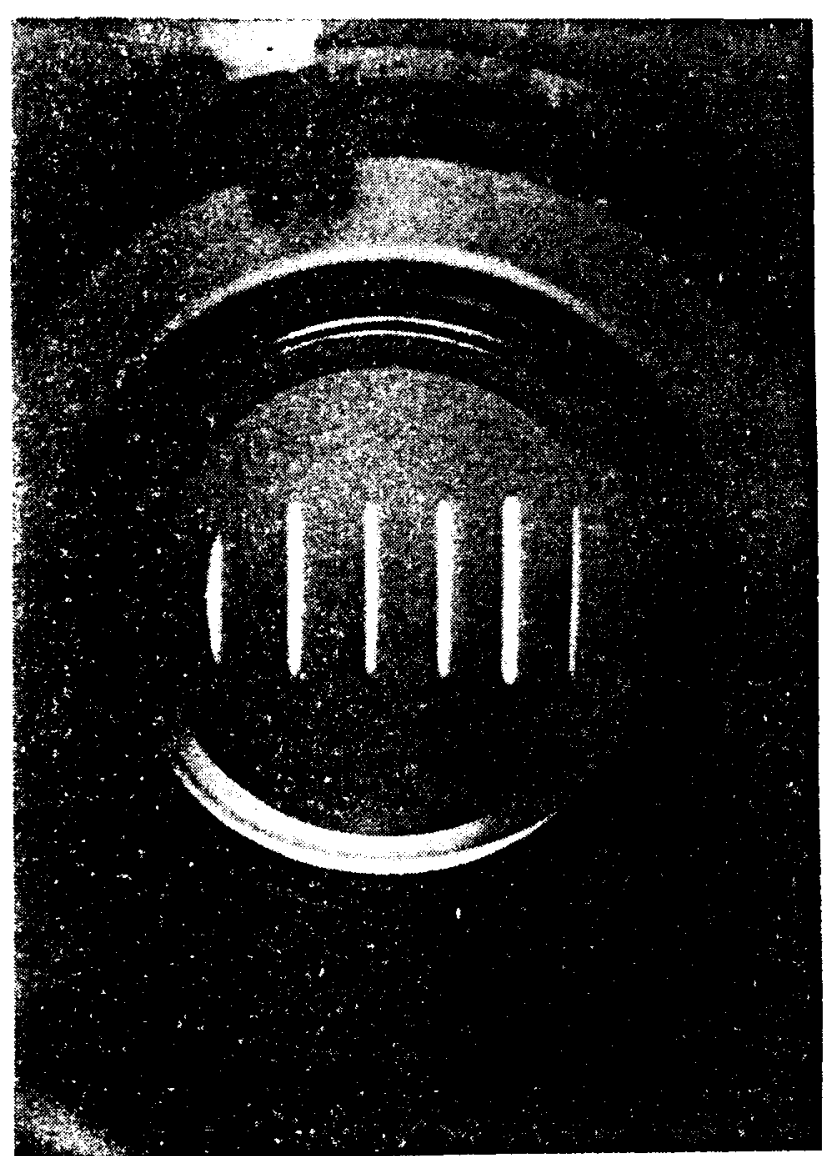

FIGURE 4. Photograph of Operating TDAPhosphor Screen (Mass Range covered is from 40 to 45 AMU). 


\section{GAIN MEASUREMENTS}

Incident ion current was measured by steering the ion beam to a Faraday cup collector placed in the beam access port of the detector housing, as shown schematically in Figure 2. An electron trap was added after noting that unwanted secondary electrons, due to collisions of the ions with the tube walls, could enter the Faraday cup with adverse effects on current readings. Since the cup entrance aperture does not lie on the focal plane of the instrument, its width was set at $2 \mathrm{~mm}$ to insure collection of all ions at a particular mass. The electron current from the MCP was measured using the phosphor screen as the electron collector. Initial gain measurements were made using the hydrogen present as residual gas in the vacuum system so that only one mass would be incident when the beam was directed back to the center of the MCP. Current measurements were made with Kiethly 610-B electrometers. Current densities were estimated by measuring the area of photographed slit images produced on the phosphor screen. The slit image dimensions were controlled by tuning of the ion source of the instrument. It is important to note that maximum current transfer to the Faraday cup did not correspond to the finest slit image. Tuning was done entirely by visual observation of the shape of the slit image, adjusting ion lens potentials for maximum sharpness on the phosphor screen. Slit dimensions were nominally $14 \mathrm{~mm} \times 0.72 \mathrm{~mm}$; the current for a particular mass was therefore confined to an area of about 0.1 $\mathrm{cm}^{2}$.

Typical results for gain measurements using 3000-eV $\mathrm{H}_{2}^{+}$ions are shown in Figures 5 and 6 . Figure 5 is a plot of the natural logarithm of the observed gain versus the TDA-MCP operating voltage for incident current densities which differ by about two orders of magnitude $\left(1.29 \times 10^{-13} \mathrm{~A} / \mathrm{cm}^{2}\right.$ and $1.18 \times 10^{-11} \mathrm{~A} / \mathrm{cm}^{2}$ ). Saturation is evident in the curve for the higher current density. The saturation effect is more noticeable in Figure 6 , the current transfer characteristics observed for $3000-\mathrm{eV} \mathrm{H}_{2}^{+}$ions. Also shown on Figure 6 are lines of constant gain; marked deviation from constantgain response occurs for currents above $10^{-12} \mathrm{~A} / \mathrm{cm}^{2}$ and voltages above $1400 \mathrm{~V}(700 \mathrm{~V}$ per MCP $)$. 


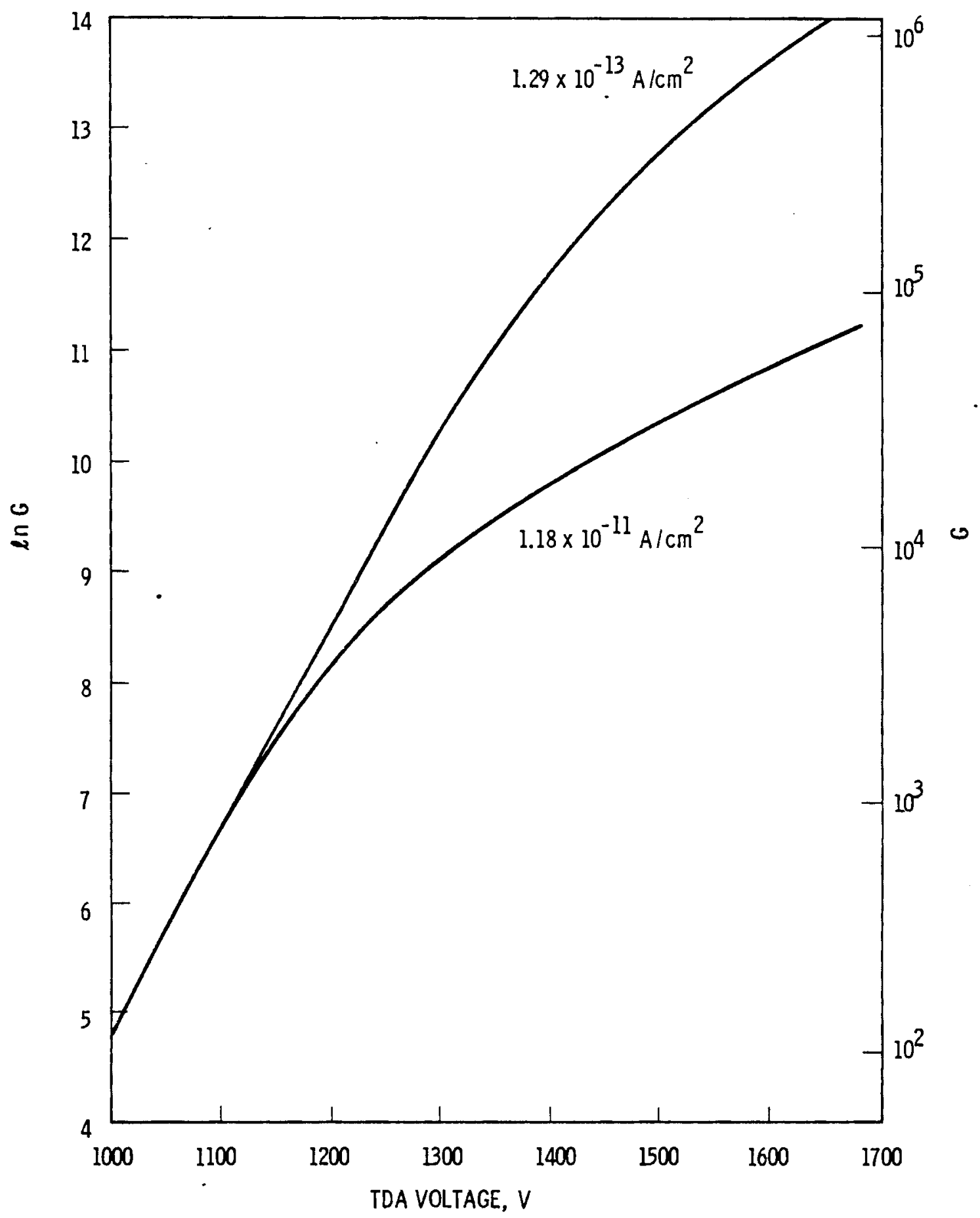

FIGURE 5. Observed TDA Gain for 3000-eV $\mathrm{H}_{2}^{+}$Ions for Indicated Currents (Left Ordinate: Natural Logarithm of the Gain, Right Ordinate: Gain Value. Abscissa: TDA Voltage; the voltage per MCP is onehalf the indicated value.) 


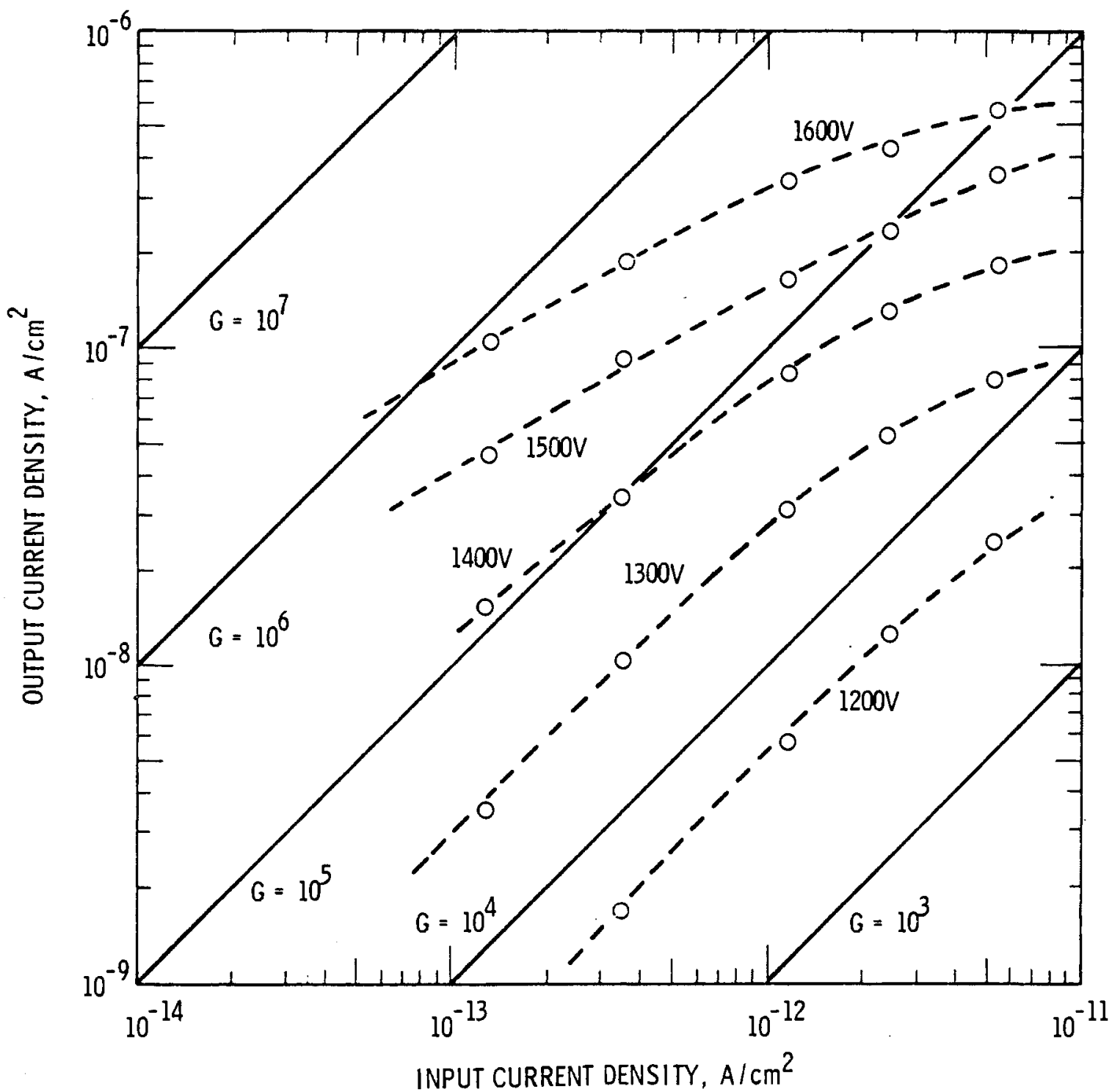

FIGURE 6. TDA Current Transfer Characteristics for 3000-eV $\mathrm{H}_{2}^{+}$Ions

Saturation appears to be more severe for $4000-\mathrm{eV} \mathrm{Kr}^{+}$ions, as shown in the curves of Figure 7 . The total current delivered to the MCP was estimated by measuring the ${ }^{84} \mathrm{Kr}$ signal in the Faraday cup and assuming the standard $\mathrm{Kr}$ isotopic distribution to calculate the curent contribution by the other isotopes allowed to reach the MCP. The total electron current delivered to the phosphor screen was then measured to determine the gain. Figure 7 compares the gain observed at $2.2 \times 10^{-14} \mathrm{~A} / \mathrm{cm}^{2}$ incident current density to that for $2.1 \times 10^{-11} \mathrm{~A} / \mathrm{cm}^{2}$. Saturation to the extent of gain reduction by two orders of magnitude is evident. 


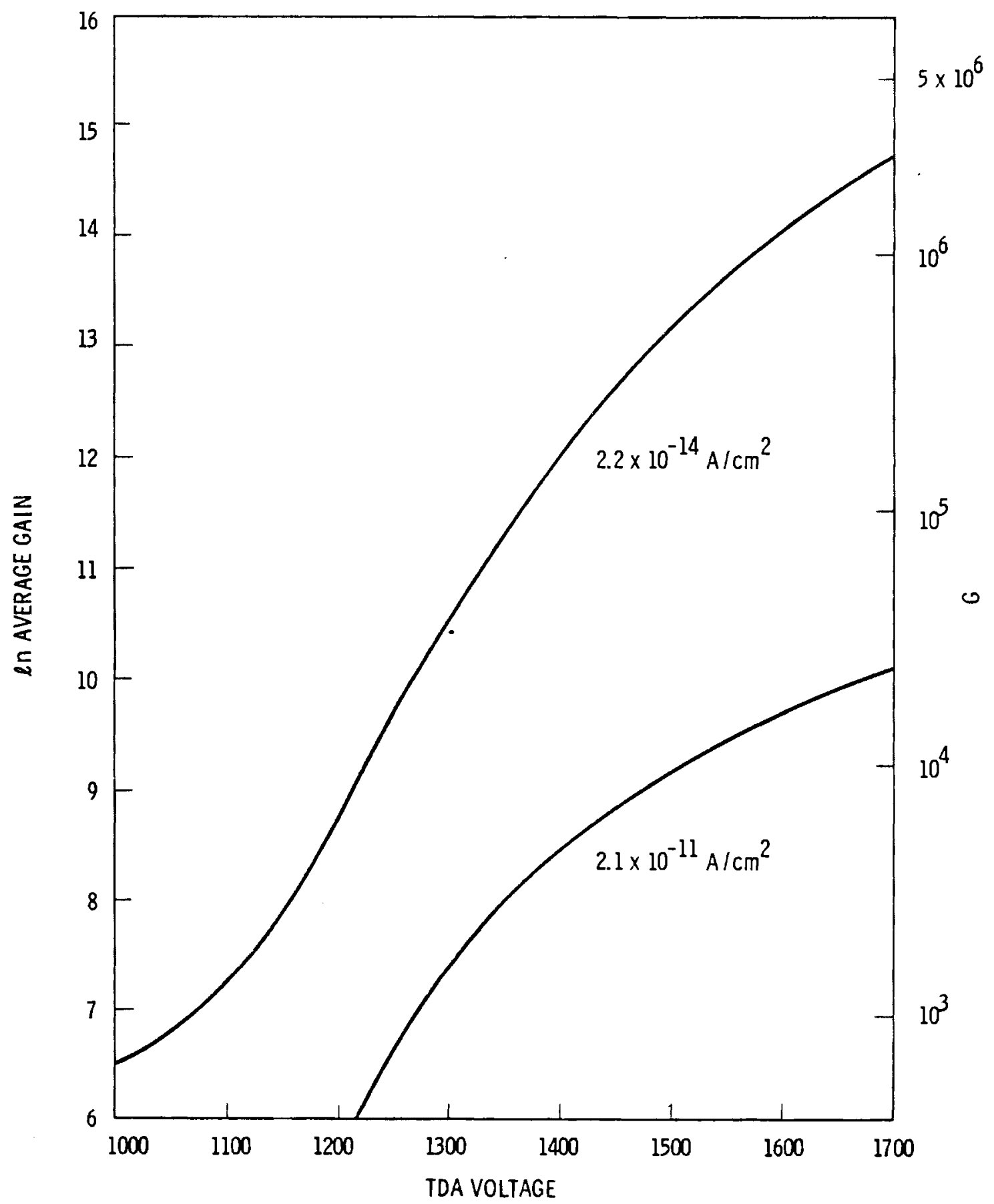

FIGURE 7. Observed TDA Gain for Krypton Isotopes at Indicated Currents 
The origin of the saturation is to be found in the magnitudes of the observed output currents. The upper lirnit of the dynamic range of the MCP is determined by the standing current on the wall of each channel, referred to as the strip current for the device. The maximum strip current is the same for a single-stage device as for a TDA. The strip current limitation becomes important when the voltage per MCP exceeds about $700 \mathrm{~V}$. Above that voltage, secondary electrons emerging from the channels raise the potential of the channel wall, thus reducing the local electric field and preventing increased gain. The TDA used in these experiments has a strip current of about one microampere per square centimenter of active surface area. The output current must not exceed $5 \%$ of this value if linear gain is to be maintained. (9)

For the TDA the output of the first MCP serves as the input for the second, so that saturation effects will occur at smaller input current densities for TDAs than for single-stage MCPs. Assuming a gain of $10^{3}$ per MCP at a bias of $700 \mathrm{~V}$ per MCP $\left(V_{C}=1400 \mathrm{~V}\right.$ for a TDA $)$, the maximum output current density for linear gain in a TDA would be about $5 \times 10^{-8} \mathrm{~A} / \mathrm{cm}^{2}$. Thus saturation is to be expected at an input current density above $5 \times 10^{-14} \mathrm{~A} / \mathrm{cm}^{2}$. This is clearly evidenced in Figures 5, 6 and 7. For an image area of $0.1 \mathrm{~cm}^{2}$ typical of the present mass spectrometer, the maximum ion current at one mass for linear gain is therefore only about $5 \times 10^{-15} \mathrm{~A}$. The upper curves in both Figures 5 and 7 are for input currents near the limit for linear gain. The difficulty of measuring currents below that level prevented extension of data to lower values of input current.

Uniform gain distribution across the entire area of a distributed detector is important to its satisfactory operation. A plot of output current as a function of peak position on the TDA for two different levels of $\mathrm{H}_{2}^{+}$incident current and at $6000 \mathrm{eV}$ and $8000 \mathrm{eV}$ is shown in Figure 8. A marked deviation from uniformity is noted. The nonuniformity is thought to be due to the fact that this TDA was used primarily for learning purposes and had already suffered significant damage prior to these measurements. Familiarization with the operation of the TDA as well as fine tuning of the image to determine magnet location for the mass spectrometer required a significant amount of operation time. Greatest damage was probably introduced by 


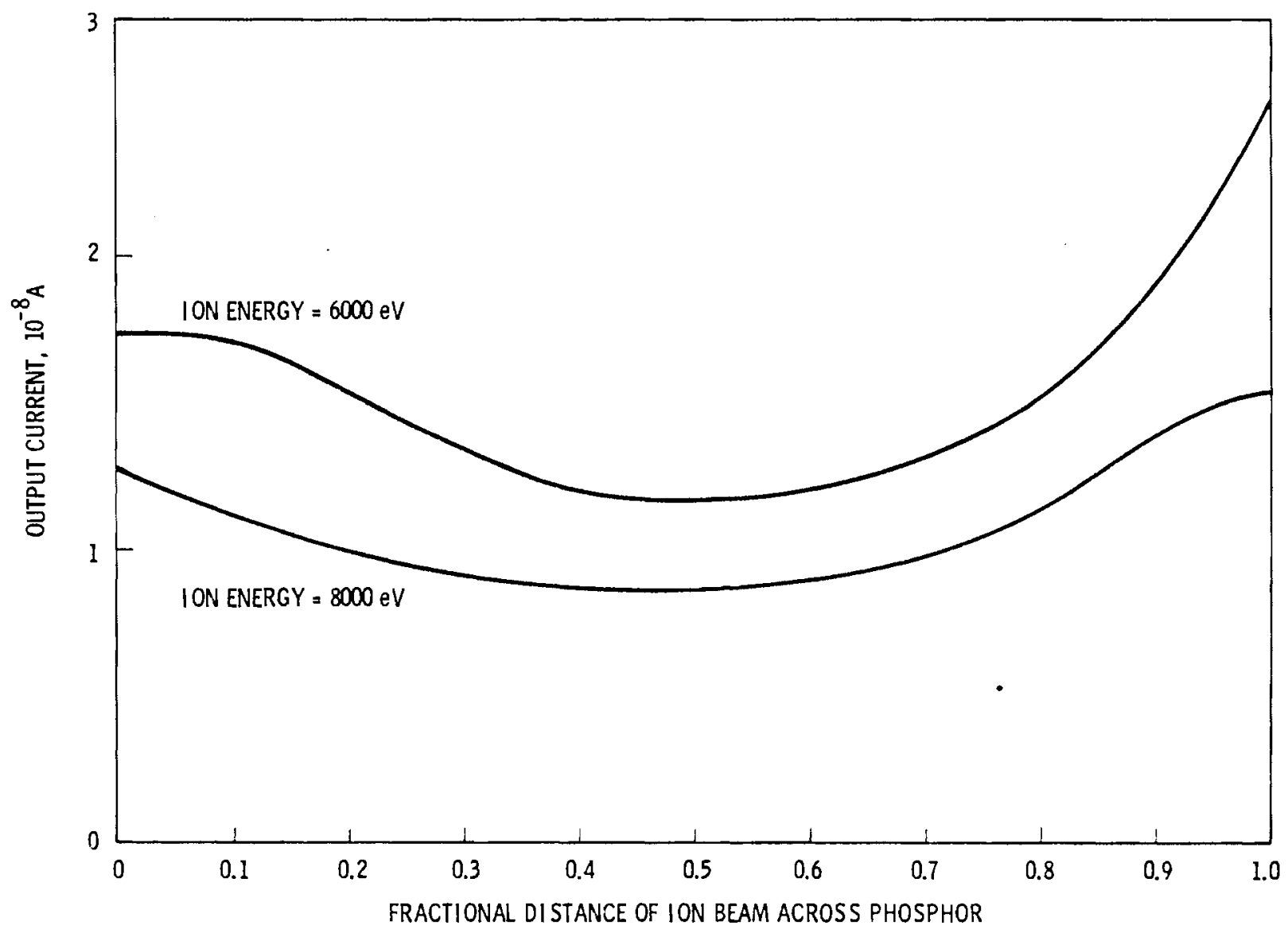

FIGURE 8. Output Current as a Function of Position of the Incident Beam on the Face of the TDA, $H_{2}^{+}$Ions at the Indicated Energies [Incident current for the $6000-\mathrm{eV}(8000-\mathrm{eV})$ curve was $9.7 \times 10^{-14} \mathrm{~A}$ $\left.\left(5.6 \times 10^{-14} \mathrm{~A}\right)\right]$

experiments in post-acceleration of ions which produced significant sputtering of the front face of the MCP. A reduction in gain by as much as a factor of two has been reported for operation times as low as 1500 hours with a $V_{C}$ of $1000 \mathrm{~V}$ and an input current density of $3 \times 10^{-12} \mathrm{~A} / \mathrm{cm}^{2}$ of low energy electron current. ${ }^{(9)}$ No accurate record of total exposure for the TDA used in these experiments was kept, so total exposure data are not available. It is likely that deterioration would be accelerated by exposure to high-energy ions. 
From results of gain measurements it is obvious that care must be exercised in the use of TDA-MCPS for focal plane detection. TDAs will always be useful qualitative tools for distributed detection of ions. Saturation at low incident current levels 1 imits their linear dynamic range so that reliance on them for accurate numerical analys is over extended current ranges is not warranted. A problem to be subsequently discussed is the non-linear gain distribution across a single mass peak due to saturation phenomena. Since the gain for incident currents below $5 \times 10^{-14} \mathrm{~A} / \mathrm{cm}^{2}$ can be several orders of magnitude greater than that for incident currents above about $10^{-12} \mathrm{~A} / \mathrm{cm}^{2}$, an artificial peak broadening on the phosphor screen can result. This would have deleterious effects on mass resolution and abundance sensitivity. One other factor, the relatively strong dependence of MCP gain on ion mass as reported by Tuithoff et al., (10) must also be considered when using these devices for accurate numerical measurements.

\section{READING AND RECORDING OPTICAL SIGNAL}

The problem of recording the signal produced by the phosphor was approached by employing a Fairchild Charge Coupled Device (CCD). The device chosen for evaluation was the CCD 121H, an array consisting of $1 \times 1728$ photodiodes, each of which is $13 \mu \mathrm{m}(0.51 \mathrm{mils})$ by $17 \mu \mathrm{m}(0.67 \mathrm{mils})$ on $13-\mu \mathrm{m}$ centers. There is no dead area throughout the $2.2-\mathrm{cm}(0.88-i n$.$) active length$ of the device. The image sensor elements are separated on the single crystal silicon chip by diffused channel stops and covered by a polycrystalline silicon photogate. Photons pass through the photogate and are absorbed in the single crystal silicon by hole-electron pair production. Electrons thus generated are accumulated in the photosites. The amount of charge accumulated is proportional to the illumination intensity and the total time of exposure, or the integration period. The background level in the device is determined by thermally gerierated noise, and the saturation exposure of nominally $0.5 \mu \mathrm{J} / \mathrm{cm}^{2}$ determines maximum output. The CCD is mounted on a vacuum-enclosed thermoelectric cooling system capable of operating temperatures to $-50^{\circ} \mathrm{C}$. (Marlowe Industries SP1056 in the Marlowe ST1018 vacuum enclosure. Marlowe Industries, Garland, Texas.) 
On-chip circuitry permitting read-out of the stored charge includes tiwo transfer gates, two 366-bit analog shift registers, and a gated charge detector/preamplifier. The transfer gate structures are adjacent to the row of image sensor elements and serve to transfer the accumulated charge to the shift registers, with alternate charge packets transferred to the right or left shift registers. The two shift registers are used to move the imagegenerated charge packets serially from the sensor elements to the charge detector/preamplifier. The phase relationships of the two shift registers provide for alternate delivery of charge packets to re-establish the serial sequence of the photosites. The charge detector/preamplifier is a precharged diode whose potential changes linearly in response to the quantity of signal charge delivered. This potential is applied to the gate of an n-channel MOS transistor which produces the final output signal. The charge detector diode capacitance is recharged by a reset transistor driven by a reset clock operating at the frequency with which packets are delivered to the detector so that the original charge state is established prior to the arrival of each new signal charge packet.

The various voltages and clock waveforms required for operation of the CCD were obtained with the CCD Design Development Board, Faircinild part no. SL62912. The board is driven by an external power supply providing a logic voltage of $5 \mathrm{~V}$ at $0.5 \mathrm{~A},+15-\mathrm{V}$ bias at $150 \mathrm{~mA}$, and $-15-\mathrm{V}$ bias at $40 \mathrm{~mA}$. A Hewlett-Packard model 222A pulse generator was used to provide the clock frequency so that a range in operating rates and integration times could be investigated. Also, in experiments reported here, the video output of the device was fed to a sample and hold amplifier so that only peak values during the measurement of each charge packet could be recorded. This greatly improved the qualitative nature of the output trace on the oscilloscope, as shown in Figure 9, where the video and sample and hold output are compared. Total time available for digital sampling of each signal was also increased by use of the sample and hold circuit. Positive spikes seen in the video output are due to feed-through from the reset pulse applied to the charge detector/preamp. The reader is referred to Fairchild Semiconductor product descriptions for further detail on the operation of the CCD $121 \mathrm{H}$. 
(a)

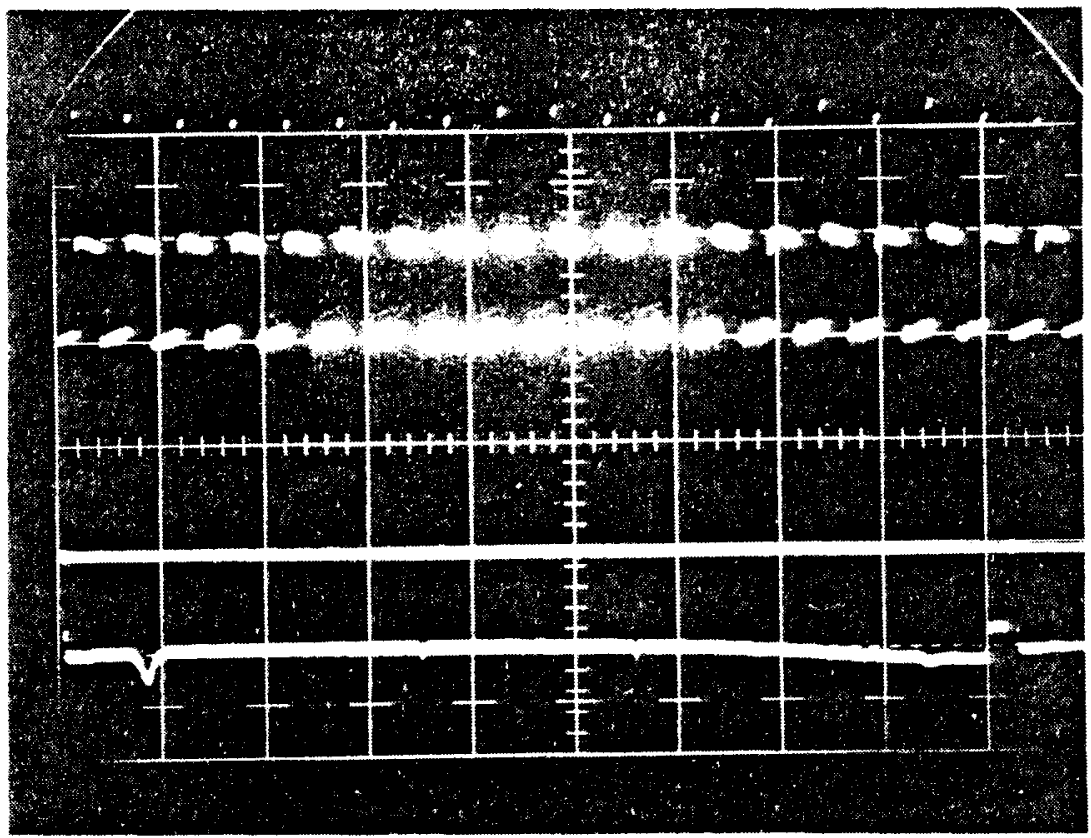

(b)

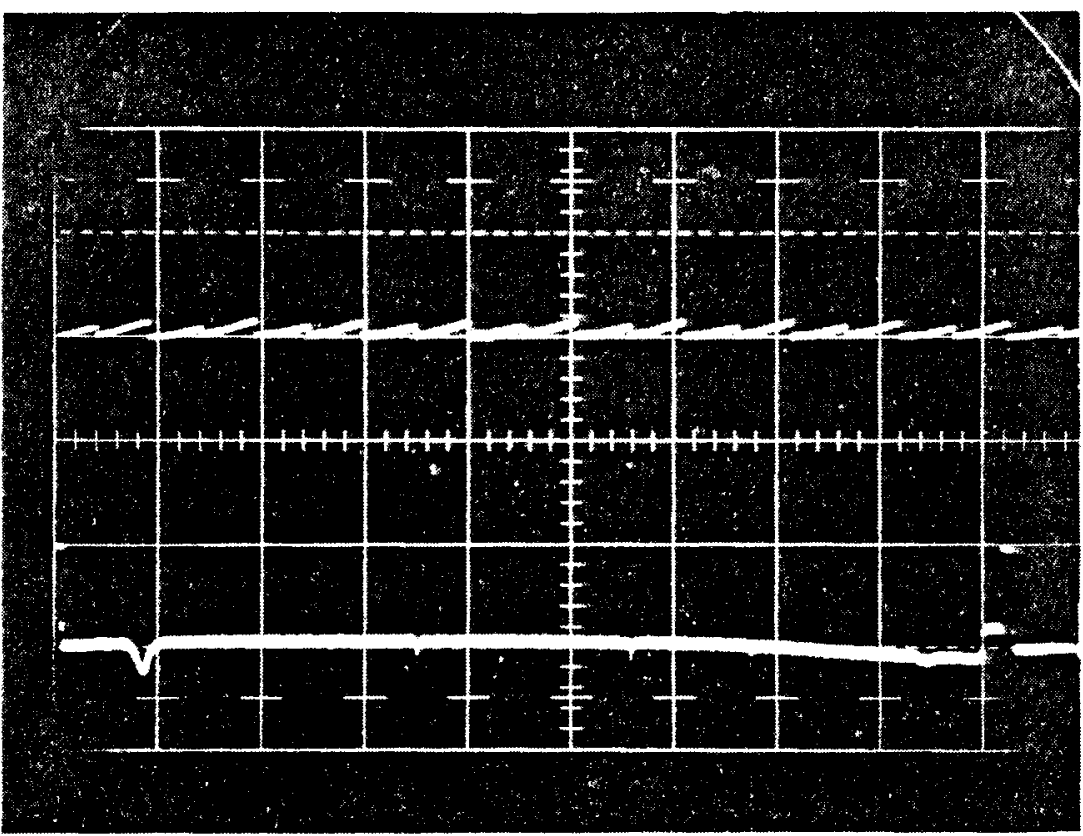

FIGURE 9. Comparison of Video (a) and Sample-and-Hold (b) Output of CCD. Sweep Speed for Lower Traces: $50 \mathrm{~ms} / \mathrm{cm}$. Upper Traces; $0.5 \mathrm{~ms} / \mathrm{cm}$. Vertical Sensitivity: $0.2 \mathrm{~V} / \mathrm{cm}$. (Zero for the upper traces of both photos is $1 \mathrm{~cm}$ down from the top graticule line, while that for the lower traces is $2 \mathrm{~cm}$ up from the bottom graticule line. Pulses showing above the graticule in (a) are due to feed-through from the clocking pulse to the on-chip amplifier. Signals are for dark current at rcon temperature and an operating frequency of $3.84 \mathrm{kHz}$.) 
A special optical system to image the $40-m m$ active diameter of the distributed detector onto a $25-\mathrm{mm}$ strip was designed and constructed. The lens system consists of three elements. The required reduction of 0.625 is accomplished using a negative lens of $-150-\mathrm{mm}$ focal length. The virtual image of that lens is then imaged on the CCD with a magnification of unity in the horizontal direction by a cylindrical lens of $90-\mathrm{mm}$ focal length. Additional vertical reduction of 0.2 is provided by a second cylindrical lens of 50-mm focal length. The lenses are mounted in a brass tube with provision for adjustments which permit superposition of the images from the two cylindrical lenses on the CCD. The system has a F-number of about $\mathrm{f} / 7$. Estimates of collection efficiency based on enirance apertures determined by working distances indicate that approximately $0.24 \%$ of the light emitted by the phosphor is collected by the optical system. The 10 uncoated glass-air interfaces between the phosphor and the CCD each have a transmission of about $80 \%$, so that $9.5 \%$ of the collected light reaches the CCD. At the combined horizontal and vertical magnification of 0.125 , the intensity in the focal plane is about $0.18 \%$ of that on the phosphor.

\section{DATA ACQUISITION AND PEAK SHAPE}

Although suitable for qualitative observation, oscilloscopic records do not provide sufficient accuracy for quantitative measurements. Numerical data in the form of the voltage output of each photosensor in the CCD are required. For the purposes of this investigation, quantitative data were obtained with a Digital Equipment Corporation (DEC) PDP11/10 minicomputer equipped with a Laboratory Peripheral System (LPS11). The LPSII is a general-purpose system with sufficient flexibility for application to a wide variety of laboratory data acquisition and experimental control problems. Data acquisition features include a real-time clock, an A/D converter multiplexed to accept up to 16 channels of input, a D/A converter with 4 channels for output, two Schmitt triggers for synchronization of sampling with external events, and a lightemitting-diode display. The reader is referred to product literature available from DEC for more detailed descriptions of the components of the LPS11. Software available for support of the system includes MACRO, BASIC, 
and FORTRAN IV. Thus, the probiem of coilecting numerical data from the CCD was reduced to development of FORTRAN programs utilizing subroutine calls to LPS11 support elements.

Three programs were written for data acquisition, display and production of hard copy on a Gould 5000 printer/plotter. Program DIODE takes data and writes the results on a file on the RKO-5 disk. Program PKREAD retrieves data from the file, displays it on a Tektronix 4010 graphics terminal, and uses the cross-hairs on the terminal to define endpoints for numerical integration over mass peaks as well as a determination of the maximum signal level between the two endpoints. Both sets of values, i.e., the integrated and maximum values, are then used to calculate isotopic distributions for the spectrum displayed. Program GLDPLT reads data from the disk file and prints out all 1728 values corresponding to the voltage levels produced on each photosite and produces a hard-copy plot of the signal height versus diode number. Listings of the programs and brief descriptions of them are given in the Appendix.

Pertinent elements of the sampling program deserve discussion here. The dark signal on the CCD was always recorded prior to turning on the ion beam. The program was designed so that the average of as many as thirty scans of the CCD could be recorded. This required that each scan be synchronous with both the recorded dark signal and successive scans. The recorded average dark signal was then subtracted from the data prior to storing the data on the disk. Synchronization of successive sweeps is accomplished using the Schmitt triggers on the LPS11. The LPS11 real-time clock is started by feeding the transfer gate signal into Schmitt trigger \#2. The rate of the clock is determined by the frequency of the oscillator driving the CCD circuitry via Schmitt trigger \#1. The clock is stopped after one pulse of the driving signal and that event is sensed by the computer. The next transfer gate pulse to arrive restarts the clock and the sampling circuitry is enabled in a mode which samples after each clock pulse. In order to start sampling simultaneously with the start of the CCD output, a preset value is counted off prior to enabling the sampling circuitry. This allows for time required by the computer to reset flags and to accumulate successive samples in a buffer prior to taking the average of several scans. This technique guarantees synchronization between recorded samples but only records evary other sweep of the C:a. A schematic 
diagram illustrating the pertinent connections to the computer system is shown in Figure 10.

At the operating temperature of $-40^{\circ} \mathrm{C}$ the dark signal in individual photosensors of the CCD was always in the neighborhood of 46 to $60 \mathrm{mV}$ for a total integration time of $1 \mathrm{~s}$. The output signal at saturation was $0.966 \mathrm{~V}$. Signals due to light on the phosphor were apparently simply added to the dark signal. The difference between the dark signal and the signal from the phosphor was independent of the dark signal as long as the total was less than the saturation level. The observed dark signal was within the range of the $5 \mathrm{mV} / \mathrm{ms}$ specified at $25^{\circ} \mathrm{C}$ when the "rule of thumb" reduction of a factor of 2

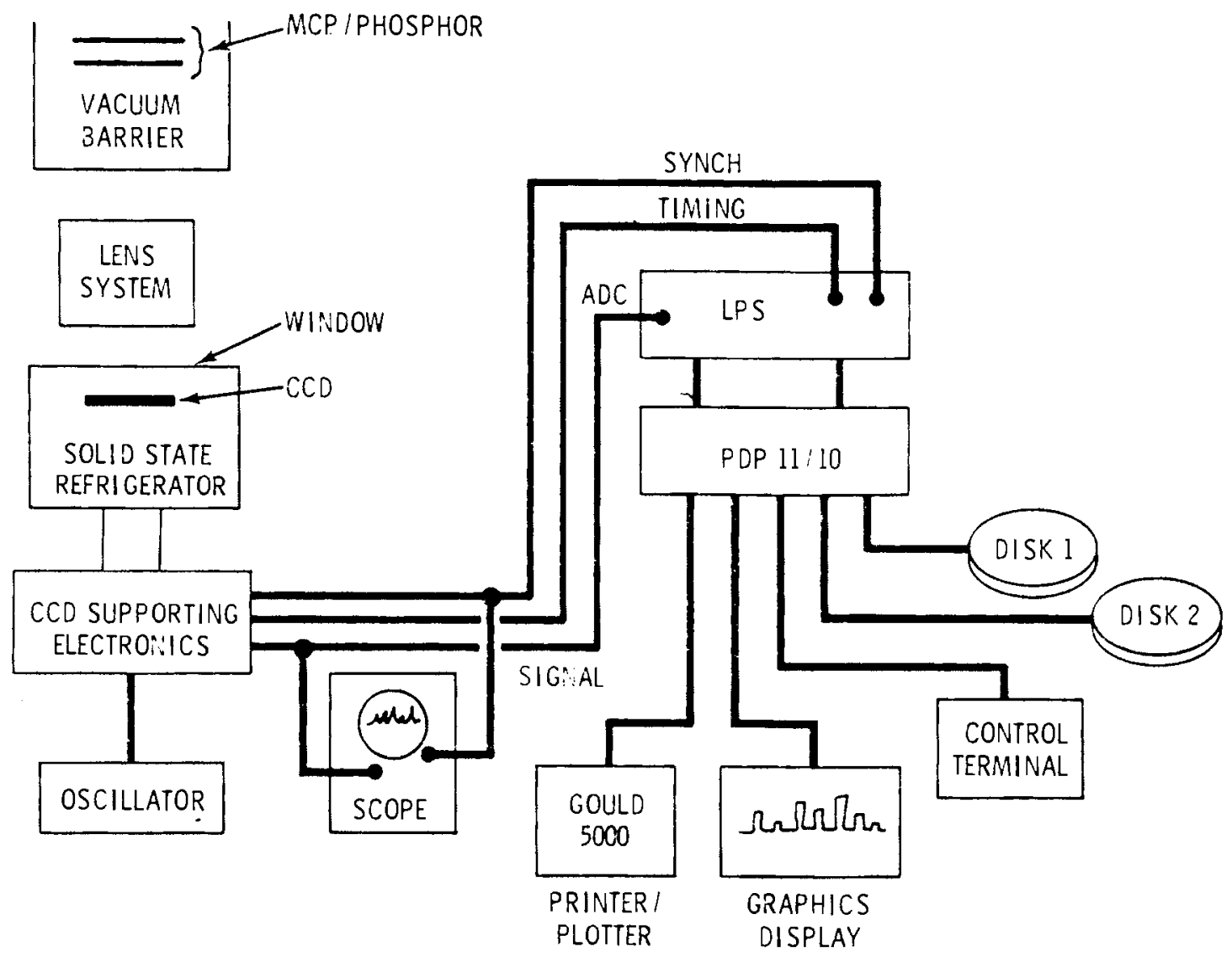

FIGURE 10. Schematic Indicating Connections Between Charge-Coupled Device and Minicomputer 
in leakage current for each $7^{\circ} \mathrm{C}$ drop in temperature for silicon devices was applied.

A typical record of the dark signal produced by the array at $-40^{\circ} \mathrm{C}$ with an integration time of $1 \mathrm{~s}$ is shown in Figure 11 whereas Figure 12 shows the output signal range. The output signal range is defined as the difference between the leakage current, or dark signal, and the saturation output. The output signal range for the device tested here is approximately $0.87 \mathrm{~V}$. Experience with numerical output of the data has shown that a difference of only 2 in output (decimal) from a 12-bit ADC (range of -1.0 to +1.0 volts) can be consistently related to the presence of a signal. The dynamic range of this device is therefore $0.87 / 0.00098=890$.

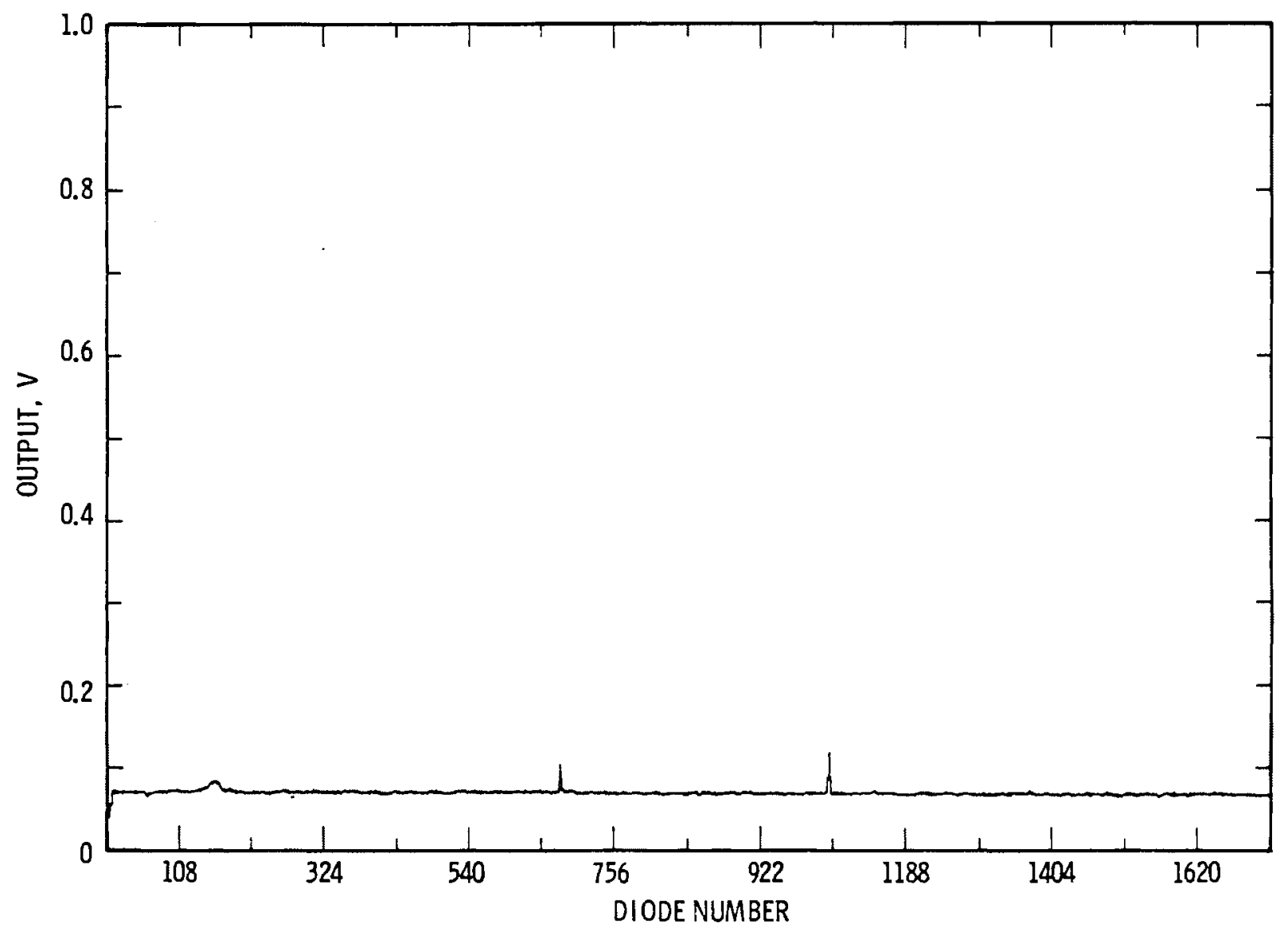

FIGURE 11. CCD Dark Signal at $-40^{\circ} \mathrm{C}$ and a Data Rate of $1785 \mathrm{~s}^{-1}$ (The integration time was $1 \mathrm{s.})$ 


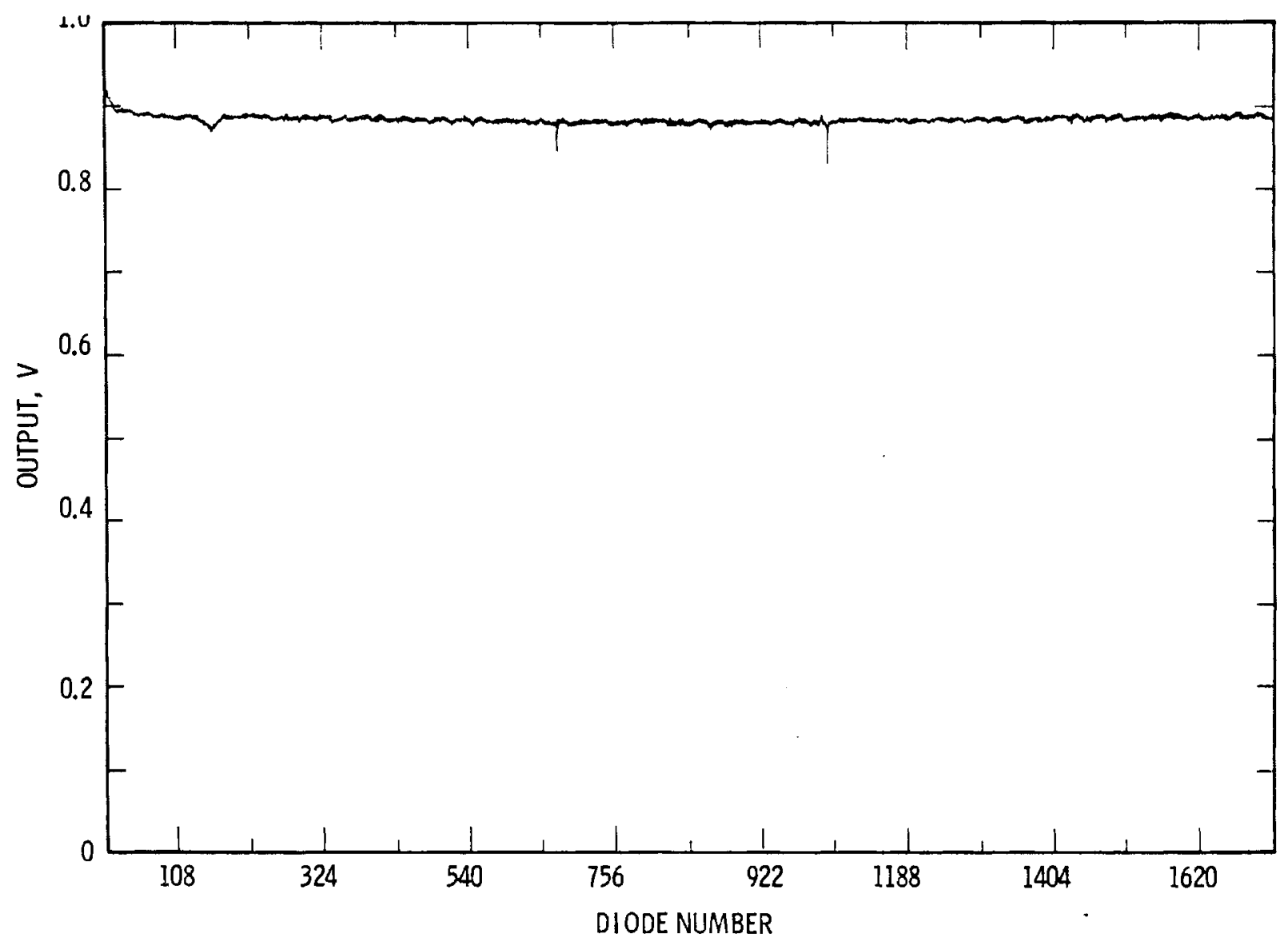

FIGURE 12. CCD Output Signal Range (i.e., the saturation output with dark signal removed)

Standard operating conditions for the TDA-Phosphor detector and the CCD were adopted for subsequent experiments. The TOA was biased at $1360 \mathrm{~V}$ with the front face at ground potential. This value is slightly below the maximum of $700 \mathrm{~V}$ per MCP which permits constant gain of a single MCP. The phosphor voltage was always set at $5000 \mathrm{~V}$ above ground. Electrons therefore reached the phosphor with an energy of $3640 \mathrm{eV}$. Integration time on the CCD was nominally $1.0 \mathrm{~s}^{-1}$. The corresponding rate of data accumulation was $1785 \mathrm{~s}^{-1}$.

The hydrogen spectrum due to residual gas in the vacuum system was used to determine the shape of an individual mass peak. Such a spectrum is shown in Figure 13 for $6000-\mathrm{eV} \mathrm{H} \mathrm{H}_{2}^{+}$ions at an incident current density of $1 \times 10^{-11} \mathrm{~A} / \mathrm{cm}^{2}$. The peak exhibits a sharp central portion with a 


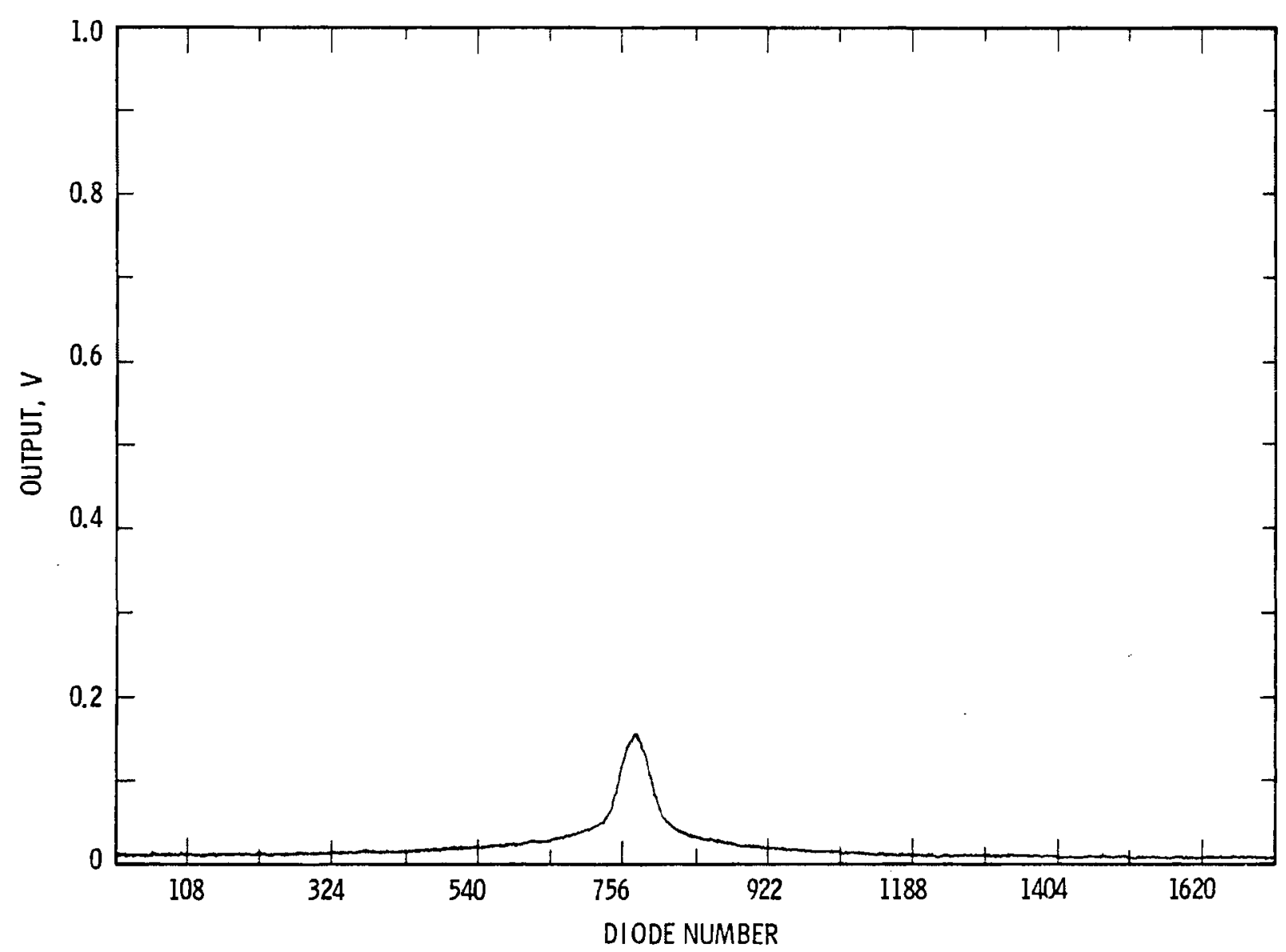

FIGURE 13. $H_{2}^{+}$Spectrum (Ion Energy $=6000 \mathrm{eV}$; Current Density $=1 \times 10^{-11} \mathrm{~A} / \mathrm{cm}^{2}$ )

maximum CCD output of $155 \mathrm{mV}$ superimposed on a broad pedestal. The central 256 points of the normalized, smoothed spectrum of Figure 13 is shown in Figure 14 to permit better visualization of the peak shape. The sharp central region of the peak has a full width at half-maximum of 61 diodes, which corresponds to $0.79 \mathrm{~mm}$ on the CCD. Since the width of the peak on the phosphor screen is $0.72 \mathrm{~mm}$, it is apparent that the optical system does not produce the expected 0.625 horizontal magnification of the image. Slight misplacement of the lenses is probably responsible for the increased width.

The central portion of the spectrum strongly resembles tiwo superimposed Gaussian curves. A Gaussian analys is of the spectrum is shown in Figure 15 , a plot of $\ln (F)$ versus $\left(N-N_{0}\right)^{2}$, where $F, N$, and $N_{0}$ represent signal height, diode number, and peak center, respectively. Two straight line portions are 


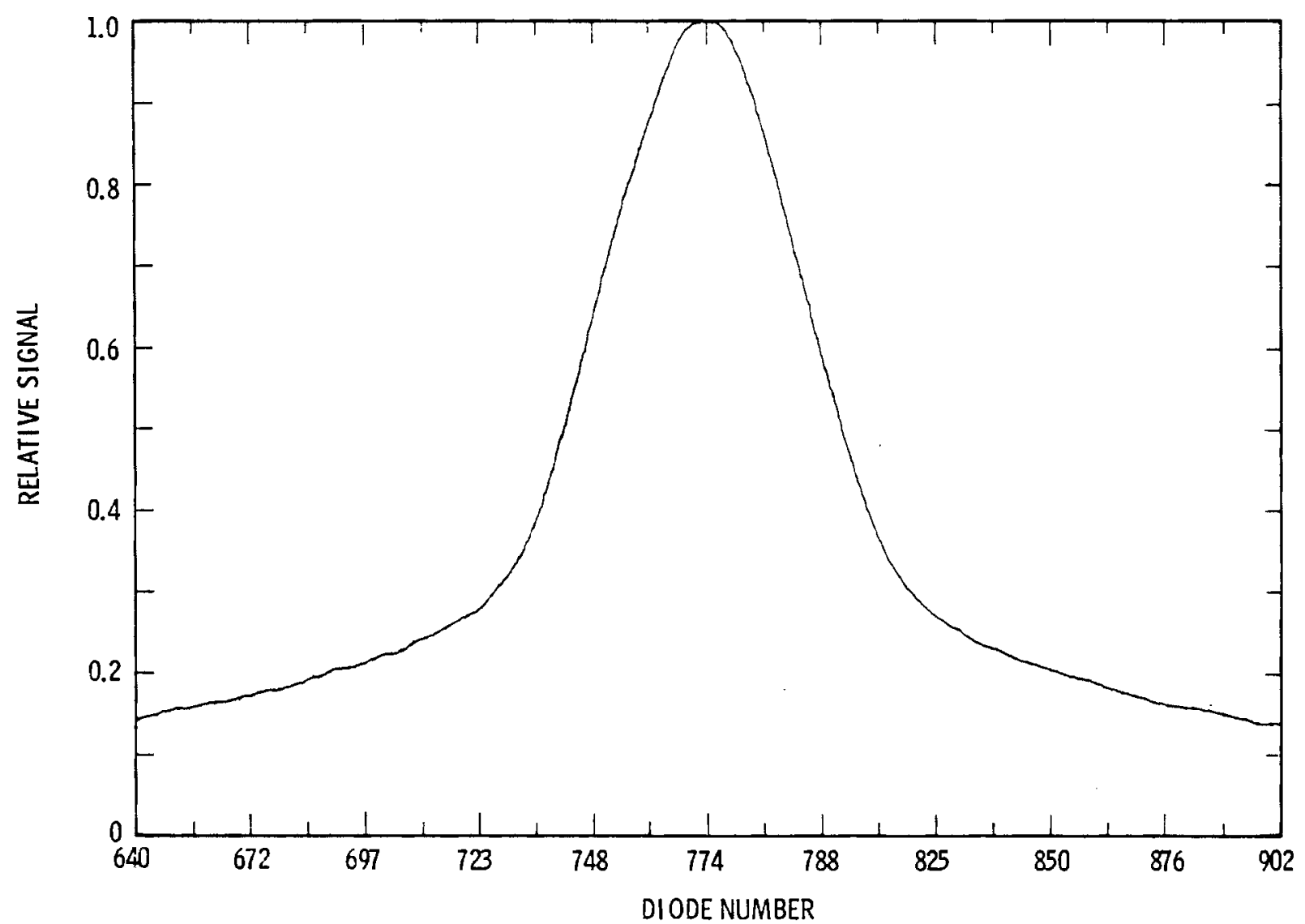

FIGURE 14. Central 256 Points of the Spectrum of Figure 13

apparent. The sharp central region has a center at diode 774 and a standard deviation of 51 diodes, while the broad pedestal shows a satisfactory Gaussian character with a center at diode 772 and a standard deviation of 115 diodes. Using the diode separation of $0.013 \mathrm{~mm}$, the full width at the $1 / \mathrm{e}$ point on the sharp portion is $0.937 \mathrm{~mm}$, while that for the broad portion is $4.2 \mathrm{~mm}$. The latter width cannot be explained solely on the basis of misplacement of optical components.

Comparison of normalized spectra obtained using $72-\mathrm{eV}$ and $135-\mathrm{eV}$ electrons in the ion source indicate that the shape of the broad pedestal is independent of electron energy. Further, an estimate of peak width made by sweeping the $\mathrm{H}_{2}^{+}$beam over the edge of the Faraday collector entrance aperture yielded $0.71 \mathrm{~mm}$, a value in agreement with the $0.72 \mathrm{~mm}$ measured on the 


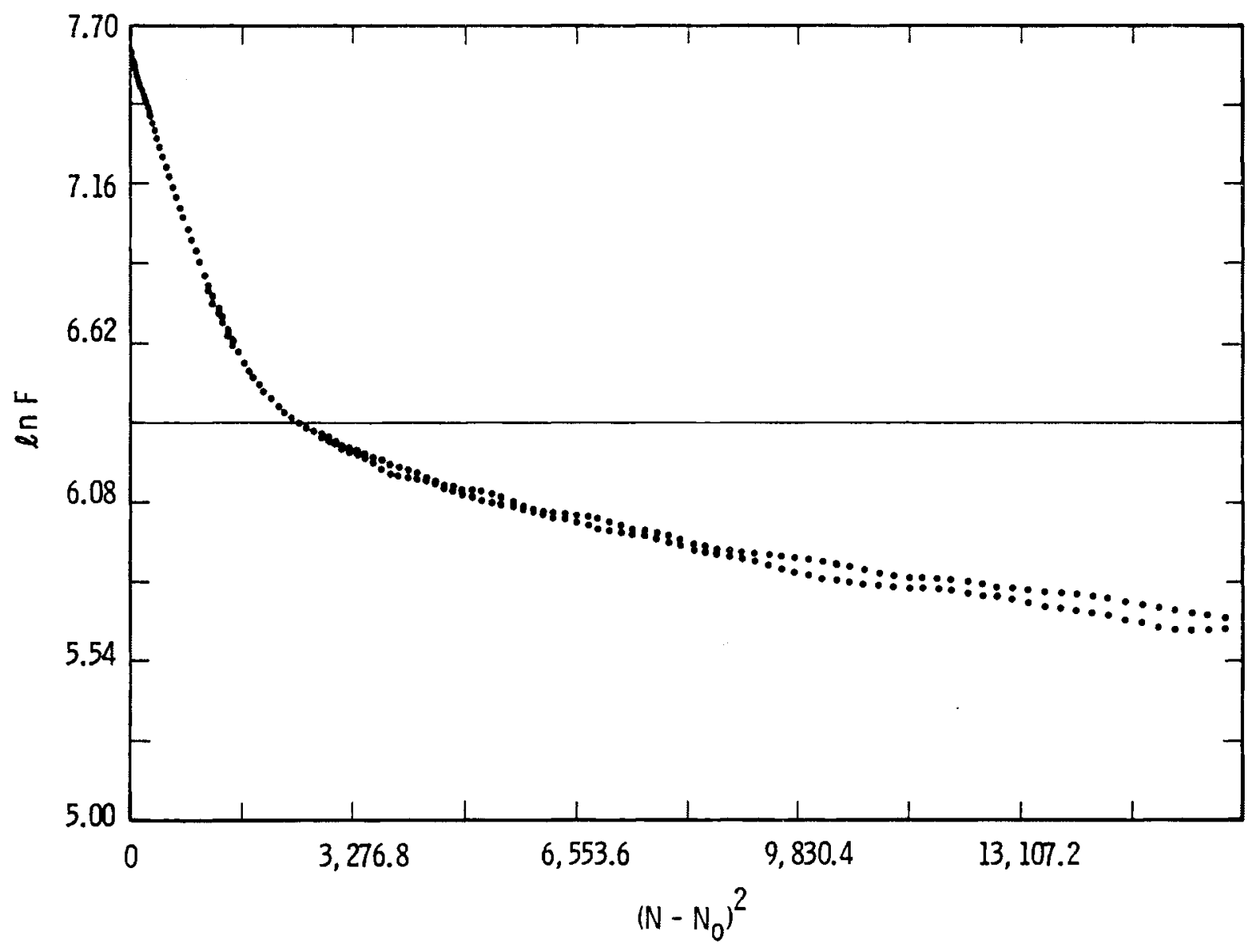

FIGURE 15. Gaussian Analys is of Figure 14

face of the phosphor. The shape characteristics are most likely the result of nonuniform gain distribution across the mass peak. In the center of the spectrum the incident current density of $1 \times 10^{-11} \mathrm{~A} / \mathrm{cm}^{2}$ exceeds the saturation limit for the TDA. The gain in that position is therefore in the neighborhood of $10^{4}$. Currents in the wings of the Gaussian pedestal are due to ions scattered from the walls of the system and are well below the limiting current for constant gain. The gain for these currents can therefore exceed $10^{6}$, with the consequence that the light produced by the phosphor per ion striking the TDA is two orders of magnitude greater in the wings of the peak than it is in the center. 
The effect of the broad peak shape on spectral quality is illustrated in the $\mathrm{Kr}$ spectrum of Figure 16. That spectrum was obtained at a pressure of $7.05 \times 10^{-6}$ torr; the ion current for ${ }^{84} \mathrm{kr}^{+}$was $6.92 \times 10^{-12}$ A so that the incident current density of $6.92 \times 10^{-11} \mathrm{~A} / \mathrm{cm}^{2}$ was well above the limit for constant gain. Integration time on the CCD was $1.033 \mathrm{~s}$. This pressure was approximately two orders of magnitude above the background, so that effects due to residual gases would be minimized. The broad contribution to the spectrum due to scattering prevents the signal from reaching the background leakage level at any position on the CCD, and is most noticeable in the 82,83 , and 84 mass peaks. Superposition of the wings of these peaks produces a valley between them with an amplitude greater than the total height of the mass 80 signal. The TDA saturation effects in the sharp portions of the peaks are such that neither peak height nor area measurements produce the correct isotopic distribution for $\mathrm{kr}$.

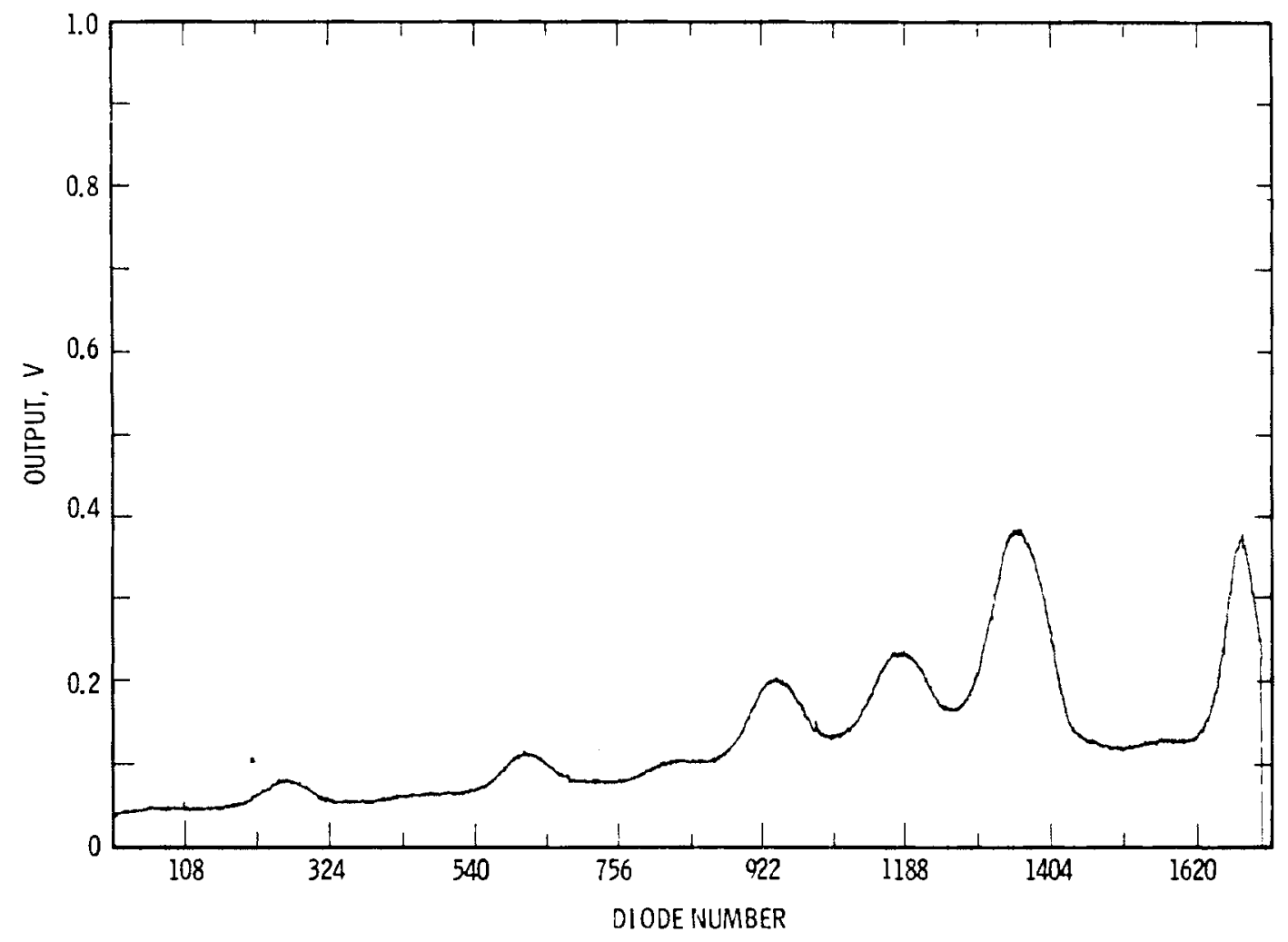

FIGURE 16. Krypton Spectrum: Masses 78, 80, 82, 83, 84, and 86 (Contributions due to residual gases at intermediate mass positions are evident.) 
The known isotopic distribution can be used to estimate the relative gain at different mass positions. Let $R_{i j}$ denote the known isotopic abundance ratio, $S_{i}$ the CCD voltage, and $G_{i}$ the gain for mass $i$. Then the relative gain can be defined as

$$
\frac{G_{i}}{G_{j}}=\frac{1}{R_{i j}} \frac{S_{i}}{S_{j}}
$$

Results for the spectrum of Figure 16 are shown in Table 1.

In substantial agreement with the current transfer characteristics of Figure 6, the appropriate gain for mass 78 with an incident current density of $4.3 \times 10^{-13} \mathrm{~A} / \mathrm{cm}^{2}$ is more than one order of magnitude greater than that for mass 84 .

TABLE 1. Gain Relative to Mass 84

\begin{tabular}{|c|c|c|c|}
\hline Mass & $r_{1,84}$ & $\underline{S_{j}, \text { Volts }}$ & $\underline{G_{i} / G_{84}}$ \\
\hline 78 & 0.00615 & 0.081 & 34.54 \\
\hline 80 & 0.0399 & 0.114 & 7.52 \\
\hline 82 & 0.203 & 0.200 & 2.58 \\
\hline 83 & 0.203 & 0.229 & 2.96 \\
\hline 84 & 1.000 & 0.381 & 1.00 \\
\hline 86 & 0.305 & 0.371 & 3.19 \\
\hline
\end{tabular}

\section{DETECTION SENSITIVITY}

The object of experiments conducted was to determine the sensitivity of the total detector system. This required measurement of the output of the CCD as a function of the ion current striking the TDA. Incident ion current was measured by steering the beam to the Faraday cup, and the output of the CCD was measured using the developed sampling program. Numerical measurements of the CCD output as a function of ion current were made using $\mathrm{kr}$ gas. The relationship between gas pressure and cup current with constant emission parameters in the electron gun was first determined. The ${ }^{84} \mathrm{Kr}$ peak was monitored while the leak valve to the $\mathrm{Kr}$ gas bottle was cracked slightly open and the main valve to 
the diffusion pump was throttled down. Ion currents at other $\mathrm{Kr}$ isotopes were inferred using the established isotopic abundance of $\mathrm{Kr}$. A series of records of CCO output as a function of pressure were then recorded by leaving the leak valve slightly open and adjusting the main valve to the diffusion pump so that a dynamic equilibrium could establish the desired pressure in the chamber. A sample consisting of 10 successive scans was then recorded and the average, with dark signal removed, filed on the disk.

A plot showing the CCO output in volts versus ion current with atomic mass as a parameter is shown in Figure 17. Apparent saturation effects are due to large incident current densities on the TDA so that the output current exceeds the strip current for the device. No saturation of the CCD was observed. This is not surprising because of the poor optical coupling between phosphor and CCO used in these experiments.

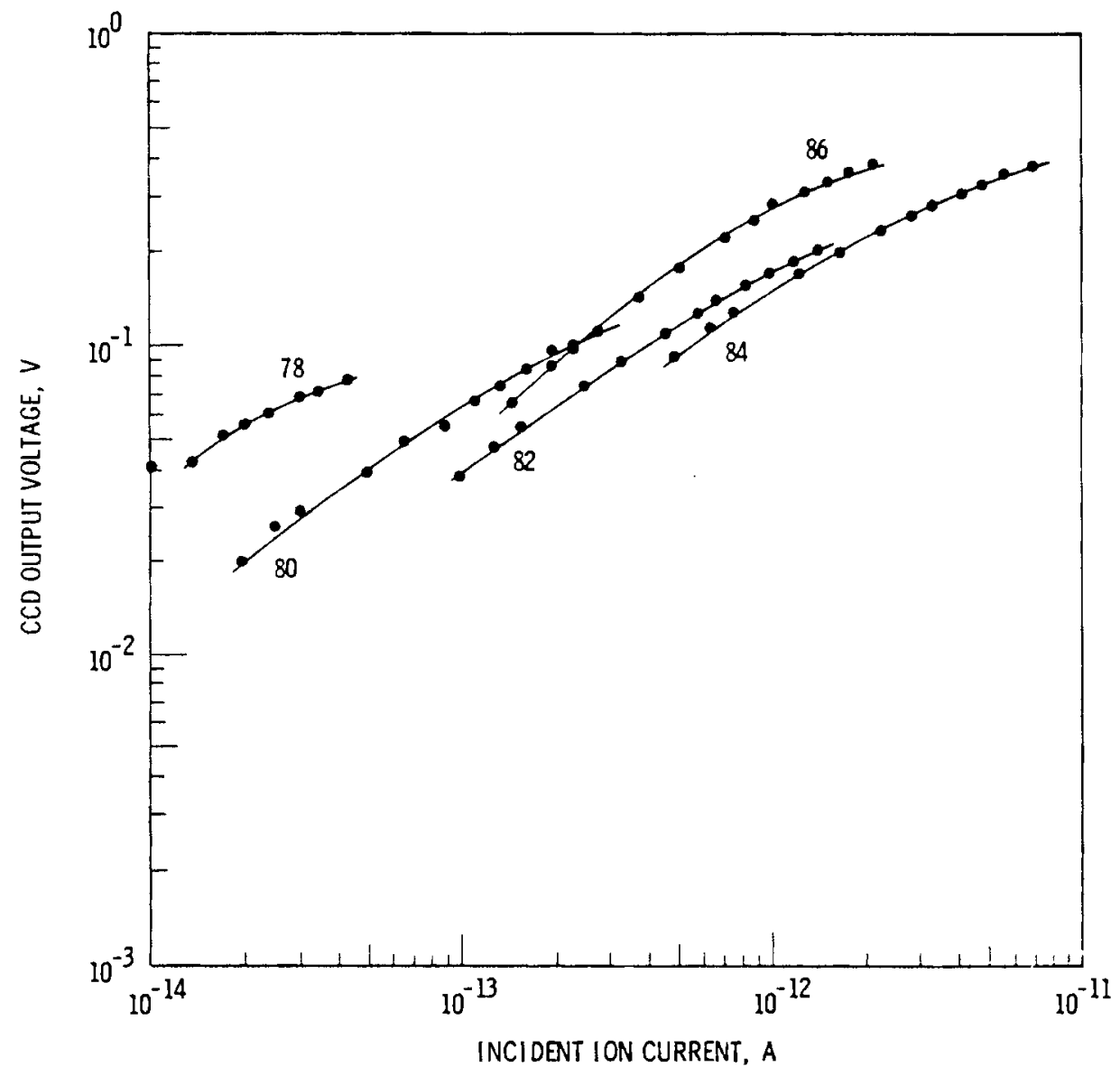

FIGURE 17. CCD Output Versus Incident Ion Current for $\mathrm{Kr}$ I sotopes 
It is seen from Figure 17 that plots of CCO output versus ion current show considerable inconsistency. This is partly due to the saturation of the MCP, previously mentioned, and partly due to assuming the current at a particular mass is due only to $\mathrm{kr}$. This is particularly true of mass 78 , which corresponds to the minor isotope of $\mathrm{kr}$ (abundance of 0.35 ) as well as to benzene $\left(\mathrm{C}_{6} \mathrm{H}_{6}\right)$. Since pressure control was achieved by throttling of the pump valve, the impurity level in the system rose along with the $\mathrm{Kr}$ pressure. Since current for each mass was not measured, corrections for background gas contributions cannot be made. Data for masses 80,82 , and 84 show similar slopes on the plot but exhibit displacement. The data for mass 86 can be explained by pointing out that the ion beam for that mass was incident on the edge of the TDA, a region which had not been exposed as much as the central portion of the TDA, and produced a brighter signal on the phosphor. (See Figure 8.)

In order to minimize questions raised by partial pressures of impurities, the determination of the ultimate sensitivity of this detection arrangement was accomplished by analys is of the 82,84 , and 86 peaks of $\mathrm{Kr}$. Figure 18 is a plot of the CCD output voltage as a function of total number of ions delivered to the front face of the MCP during the nominal 1-s integration time used in these experiments $(1.033 \mathrm{~s}$ was the actual measured value of the integration time). The relative abundances of these isotopes are $11.56 \%, 56.9 \%$, and $17.37 \%$, respectively. Saturation of the MCP is evident in all three curves. An estimate of the minimum detectable number of ions was made by making a quadratic extrapolation of the 84 signal to the baseline, or zero level of the plot. The extrapolation intersects the axis at a value corresponding to about $2.2 \times 10^{5}$ ions. The other two plots do not extrapolate to zero because of the contribution of the wings of adjacent signals to their total height.

In another independent test, straight-line fits of $V_{C C D}$ versus pressure and ion current to the Faraday cup versus pressure were compared. The ratio of the two slopes thus derived corresponds to an average sensitivity in terms of CCD output voltage per ampere striking the front face of the TDA. The pressure range for this experiment was between $1 \times 10^{-6}$ torr to $8.6 \times 10^{-6}$ torr. 


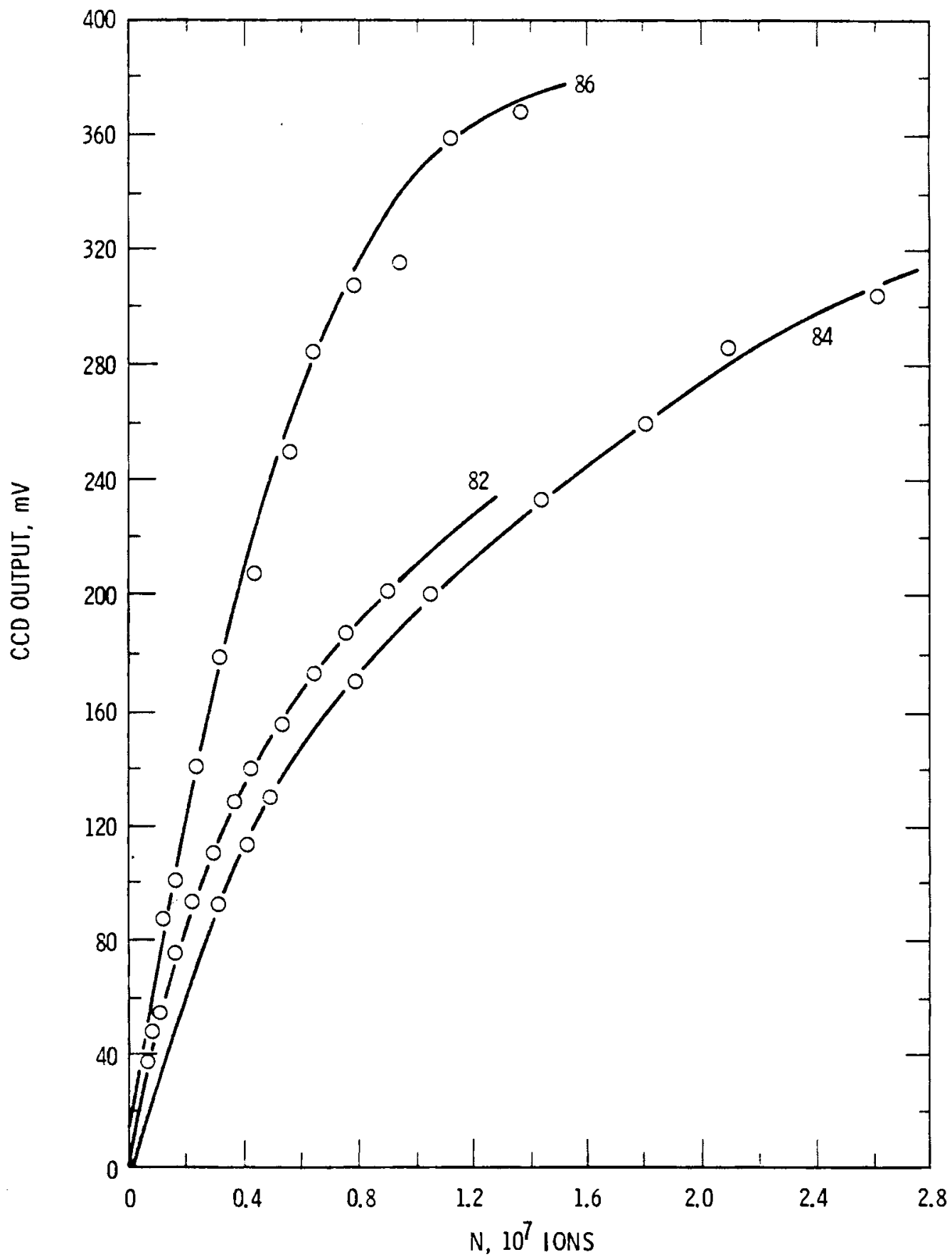

FIGURE 18. CCD Output Versus Integrated Ion Current for $\mathrm{K}_{\boldsymbol{q}}$ Isotopes 82, 84, and 86 (A quadratic extrapolation of the $84 \mathrm{Kr}$ curve yields a sensitivity value of $2.2 \times 10^{5}$ ions.) 
The following two relations resulted:

$$
I^{+}=1.732 \times 10^{-13}+7.14 \times 10^{-8} \mathrm{P}
$$

and

$$
V_{C C D}=1.805 \times 10^{-2}+1.436 \times 10^{4} \mathrm{P}
$$

with the pressure $P$ in torr for both equations. Correlation coefficients for the current and voltage lines were 0.998 and 0.982 , respectiveiy. The ratio $(d V / d P) /(d I / d P)=d V / d I$ yields a value of $2.011 \times 10^{11} \mathrm{~V} / \mathrm{A}$. The integration time employed in this experiment was $1.122 \mathrm{~s}$, so the sensitivity per ion striking the MCP derived for this case is $2.872 \times 10^{-8} \mathrm{~V} /$ ion. The sensitivity of the array is determined by the variations naturally present in the leakage signal. As can be seen from Figure 11 this varies as a function of position on the CCD. The standard deviation of the leakage signal over the entire array at the operating temperature of $-35^{\circ} \mathrm{C}$ is $2.8 \mathrm{mV}$, while the value for separate 200 element sections of the array varies between $0.8 \mathrm{mV}$ and $4.5 \mathrm{mV}$. If the value of $4.5 \mathrm{mV}$ is used as the threshold for significant signal, the sensitivity of the detection arrangement under discussion is nominally $0.0045 /$ $\left(2.872 \times 10^{-8}\right)=1.56 \times 10^{5}$ ions. This estimate agrees with that obtained by extrapolation in the previously discussed example.

\section{CONCLUSIONS AND RECOMMENDATIONS}

The most severe problem encountered in this work is that due to saturation in the TDA. It becomes important therefore to estimate the contribution of the second stage MCP to the total gain of the TDA. Published current transfer characteristics for single MCPS $(9)$ indicate that under the present operating conditions $(680 \mathrm{~V} / \mathrm{MCP})$ a gain of $1.08 \times 10^{3}$ can be expected in the first stage. Gain measurements made in this work can therefore be used as a basis for estimating the gain of the second stage. Representative data and resuits for $\mathrm{H}_{2}^{+}$ions at $8000 \mathrm{~V}$ are shown in Table 2 with the total MCP voltage at $1360 \mathrm{~V}$. 
TABLE 2. TDA Second Stage Gain for $8000-\mathrm{eV} \mathrm{H}_{2}$ Ions. Single Stage Gain $=1.08 \times 10^{3}\left(\mathrm{~V}_{\mathrm{C}}=80 \mathrm{~V} / \mathrm{MCP}\right)$

\begin{tabular}{|c|c|c|c|}
\hline$J_{\text {in, }} A / \mathrm{cm}^{2}$ & $\mathrm{~J}_{\text {out, }} \mathrm{A} / \mathrm{cm}^{2}$ & TDA Gain & 2nd Stage Gain \\
\hline $1.04 \times 10^{-13}$ & $3.3 \times 10^{-9}$ & $3.27 \times 10^{4}$ & 29 \\
\hline $1.71 \times 10^{-13}$ & $6.09 \times 10^{-8}$ & $3.46 \times 10^{4}$ & 33 \\
\hline $3.62 \times 10^{-13}$ & $1.42 \times 10^{-8}$ & $3.75 \times 10^{4}$ & 36 \\
\hline $5.97 \times 10^{-13}$ & $2.34 \times 10^{-8}$ & $3.95 \times 10^{4}$ & 36 \\
\hline $1.19 \times 10^{-12}$ & $5.01 \times 10^{-8}$ & $4.21 \times 10^{4}$ & 39 \\
\hline
\end{tabular}

The average for the five values of Table 2 indicate that the second stage gain in this current range is only $35 \pm 4$. A single MCP has a linear gain of $10^{4}$ with a bias of $900 \mathrm{~V}$ up to an input current density of $10^{-11} \mathrm{~A} / \mathrm{cm}^{2}$.

The TDA is therefore not necessary since the increase gain is not required and can lead to excessive saturation effects.

The use of fiber optic reducers in place of a lens system for optically coupling the phosphor image to the CCD can increase the transmission to a value near $90 \%$ from the $0.18 \%$ used in these experiments. The light intensity at the CCD should be a factor of 500 higher than in the present study if efficient transmission is used. If the MCP gain is adjusted to $10^{4}$, an over-al1 increase in sensitivity of about 143 can be expected by using efficient optical coupling with a single MCP.

With $90 \%$-efficient optical coupling, the upper limit of detectability will be determined by saturation in the CCD. To demonstrate this, assume that the sensitivity figure of $2.872 \times 10^{8} \mathrm{~V} /$ ion derived in this work is correct and adjust it for anticipated improvements due to better optical coupling. We also assume that the sensitivity of the CCD is $0.4 \mathrm{~V} \cdot \mu \mathrm{J}^{-1} \cdot \mathrm{cm}^{-2}$, as quoted in product literature. Combining these estimates yields a value for light reaching the CCD per ion striking the MCP of $L=1.026 \times 10^{-5} \mathrm{\mu J} / \mathrm{cm}^{2}$ ion. The output signal range for the CCD used in these experiments was shown to be $0.86 \mathrm{~V}$. This corresponds to a light intensity on the CCD of $2.15 \mu \mathrm{J} / \mathrm{cm}^{2}$. 
The maximum total number of ions which can be seen is therefore the ratio of the last two numbers, namely $2.1 \times 10^{5}$. This value is close to the minimum detected in these experiments and would therefore seem to pose a difficult quandary. However, the great flexibility in integration times for the CCD allows it to operate with variable sensitivity and maintain its linearity. The minimum detectable signal is, as previously stated, (2/2048) volts. This would correspond to 238 ions striking the single-stage MCP. The dynamic range of the system with fixed integration period of $1 \mathrm{~s}$ would therefore be 881 . The potential for dynamic adjustment of integration time on the CCD under computer control can extend its dynamic range considerably. The combination: single-stage MCP - Fiber Optics - CCD should prove flexible enough for a broad range of analytical situations.

For ion currents densities below about $10^{-14} \mathrm{~A} / \mathrm{cm}^{2}$ the TOA remains an attractive candidate for focal plane detection. However, due to saturation effects, care must be taken to design mass analyzer systems to minimize scattering in the source region and from analyzer tube walls. These conditions should be met with surface ionization sources in direct inlet systems. More experience with DIMS instruments is required to determine adequate sensitivity levels for distributed focal plane detectors. Work discussed here supports the recommendation that single-stage MCPS be used to avoid non-linearities found in TDA-MCPS. 


\section{ACKNOWLEDGEMENTS}

The author takes pleasure in acknowledging the contributions of others to this work. W. A. Mitzlaff led the author through the rather complicated operation of the CCD and provided invaluable electronics design support, including design of the sample and hold amplifier. Harry Emtman constructed several circuits used in reading of the CCD as well as special supplies for the operation of the mass spectrometer. D. R. Ells was instrumental in both design and assembly of the mass spectrometer and the detector mounting method, and G. A. Reick, Jr., laid out the detailed engineering design of the mass spectrometer and vacuum system. Construction of the mass tube was the responsibility of Tony Madsen. G. L. Slater performed many of the gain measurements, and J. E. Fager was a valuable consultant for problems with computer programming. The work profited from many detailed discussions with Dr. C. R. Lagergren. 


\section{REFERENCES}

(1) Davis, W. D. 1977. "Continuous Mass Spectrometric Analys is of Particulates by Use of Surface Ionization." Environmental Sci. Technol. 11:587 (1977), and "Continuous Mass Spectrometric Determination of Concentration of Particulates in Air by Use of Surface Ionization." Environmental Sci. Technol. 11:593.

(2) Meyers, R. L., and W. L. Fite. 1975. "Submicron and Centimicron Particulate Detection Using Surface Ionization." American Laboratory, page 23, December 1975.

(3) Lassiter, W. S., and A. L. Moen. 1974. In-Situ Mass Analys is of Particle by Surface Ionization Mass Spectrometry. NASH-TM-X-3112, November 1974.

(4) Stoffels, J. J., and C. R. Lagergren. 1976. A Direct Inlet Mass Spectrometer for Real-Time Analys is of Particulate Contaminants in Air. BNWL-SA-5645, Pacific Northwest Laboratory, Richland, Washington.

(5) Boettger, H. G. 1973. "Electro-Optical Multi-Channel Ion Detector for a Mass Spectrometer." Paper \#T-5, presented at the ASMS 21 st Annual Conference on Mass Spectrometry and Allied Topics, May 25, 1973.

(6) Giffen, C. D., H. G. Boettger, and D. D. Norris. 1974. "An ElectroOptical Detector for Focal Plane Mass Spectrometers." Int. J. Mass Spectrum. Phy. 15:437-449.

(7) See, e.g., Tuitoff, H. M., F. J. M. Boerboom and H. L. C. Meuzelaor. 1975. "Simultaneous Detection of a Mass Spectrum Using a Channel Electron Multiplier Array." Int. J. Mass Spectrum. Ion. Phys. 17:299-307.

(8) Dietz, A. 1959. "Ion Optics for the V-Type Surface Ionization Filament Used in Mass Spectrometry." Rev. Sci. Inst. 30:235-241.

(9) Ruggeri, D. J. 1972. "Microchannel Plate Imaging Detectors." IEEE, Trans. Nuc. Sci. 19: (3).

(10) Tuithoff, M. H., and A. J. H. Boerboom. 1974. "Amplification Factors of a CEMA (Channel Electron Multiplier Array) for Organic, Noble Gas and $\mathrm{H}_{\mathrm{g}}$ Ions." Int. J. Mass Spectrum. and Ion Phys. 15:105-109. 

APPENDIX

RT11 FORTRAN-IV SOFTWARE; CCD DATA ACQUISITION AND ANALYSIS 
APPENDIX

RTI1 FORTRAN-IV SOFTWARE;

CCD DATA ACQUISITION AND ANALYSIS

Software development represents a significant portion of the effort expended to perform the evaluation of the approach to simultaneous mass detection described in this report. This section serves to document the results of that effort. In addition to programs required for taking data from the CCD, several were written to perform special analyses and to draw plots. The three programs most essential to the conduct of this work will be described here: Program DIODE, the routine for recording CCD output and creating a data file on the disk; Program PKREAD which retrieves the data from the disk file, draws a plot on the Tektronix 4010 graphics terminal, and uses the cross-hairs on the terminal to define the end points for integration over mass peaks so that relative abundances can be determined; and program GLDPLT which generates a printout of the stored values for each element of the diode array and a hardcopy plot using a Gould 5000 printer-plotter. In the following a general description of each program will be followed by a FORTRAN listing and a sample of the output from each program.

\section{A.1 PROGRAM DIODE}

Program DIODE is the routine used for sampling the CCD array. The program is written in the MAIN program-SUBROUTINE structure for greater flexibility, and is designed to operate on a PDP $11 / 10$ equipped with a Laboratory Peripheral System (LPS) and 16K-word core. The COMMON element includes IBUF, dimensioned at 1785, for receiving data aquired by the LPS, and IY, also dimensioned at 1785, for accumulating successive scans prior to signal averaging. Other variables in COMMON are IDUR, dimensioned at 30, for storing the results of the HISTogram sampling element of LPS support for up to 30 
consecutive scans, and ISIZ, NCALL, ISTCHN, MODE, PRESET, and LIM which represent the total number of data points to be taken per scan, a counter for recording the number of scans taken, the input channel on the LPS used for receiving data from the $\mathrm{CCO}$, the sampling mode used in the Real Time Sampling (RTS) subrouting in LPS support software, the number of received pulses to count prior to initiating the RTS routine, and the total number of scans to be taken, respectively. Definitions and uses of the COMMON variables are summarized in Table A.1.

The main program first calls SUBROUTINE DURATE which uses the LPS real time clock operating at $1 \mathrm{kHz}$ to measure the duration of 20 successive scans of the CCD. Each scan requires 1785 cycles of the external oscillator driving the CCD Design Development board. The time between scans is measured by sensing the CCD transfer-gate pulse from the Design Development Board with Schmitt Trigger \#2 on the LPS. The average scan duration and its standard deviation are then calculated and the results typed on the terminal. since there is no way to control the start of sampling synchronously with the oscillator, the first number recorded is excluded from the average.

\section{TABLE A.1. Common Element in Program Diode}

\begin{tabular}{lrll} 
Variable & Dimension & & \multicolumn{1}{c}{ Function } \\
\cline { 2 - 2 } IBUF & 1785 & & Data receiver \\
IY & 1785 & & Data accumulator \\
IDUR & 30 & & Storage of Histogram data \\
ISIZ & 1 & Number of points in IBUF \\
NCALL & 1 & Counter \\
ISTCHN & 1 & LPS ADC channel number \\
MODE & 1 & Sampling mode of LPS \\
PRESET & 1 & Number of cycles delay between \\
LIM & 1 & Sotal number of scans to be taken
\end{tabular}


Program DIODE next calls SUBROUTINE SETUP. This is a simple initialization routine which zeroes all buffers and prompts the user with messages to the terminal. Necessary run parameters, such as the number of points to be recorded, the sampling mode, the LPS ADC channel number to be used, the number of pulses to be counted prior to the start of sampling, and the total number of scans to be recorded, are establisined in SETUP.

The background or dark signal from the CCD is next recorded with SUBROUTINE BKGRND. BKGRND calls SUBROUTINE DATAKE and SUBROUTINE PUTBKG. SUBROUTINE PUTBKG creates a file on the RKO-5 disk which contains the results of sampling in unformatted binary using routines in the DEC System Subroutine Library. SUBROUTINE DATAKE is the basic sampling element of the program and is used for all data-taking activity of the program. A detailed description of DATAKE will be given here.

For effective signal averaging, each successive scan must be in synchronization. Synchronization is established in the loop "DO $40 \mathrm{I}=1$, LIM" in the listing for SUBROUTINE DATAKE. All event flags are first initialized to 0 in the loop. The histogram routine, HIST, is then called to sense the arrival of the next CCD transfer gate pulse via Schmitt Trigger \#2 by calling the realtime clock routine, SETR such that it is driven by the external oscillator.via Schmitt Trigger \#1 (rate 6) and in the inter stimulus mode (3), with the clock preset value set at 0.0 . The next firing of ST \#2 then fills the variable ITRIG, the first argument of the call to HIST, and sets the histogram event flag. The progress of the routine is delayed by the call to LWAIT until the histogram event flag has been set. The clock must then be turned off [CALL $\operatorname{SETR}(-1,,)$,$] and started again at the externally driven frequency and in$ single interval mode and counts off the value entered for PRESET in SUBROUTINE SETUP. After the clock flag has been set, sensed by the next call to LWAIT, the Real Time Sampling (RTS) routine is called.

The argument list for the call to RTS contains 10 elements. Data are stored in IBUF, ISIZ words are to be stored, and no subbuffers are required as indicated by the unity value of the next argument. Data are entering via channel ISTCHN, and only one channel is used. The gain has previously been 
set to 1 in the subroutine, and the MODE normally used is mode 0 , sample when ST \#1 fires. The variables ICMF and IBEF are the completion/error flag and the buffer event flag, respectively. After the setting of the completion flag, sensed by the next call to LWAIT, the current value of ITRIG is stored in the array IDUR and the values read into the buffer IBUF are accumulated in the array IY by the call to subroutine FILL. The progress of sampling is monitored in real time by displaying the current value of NCALL on the 1ight emitting diode display of the LPS with the call to LED. The clock is then turned off and the process repeated LIM times. The current values for ITRIG and the histogram and clock flags are then typed on the terminal, as are the values stores in IDUR, representing the number of oscillator cycles between clock start and the next transfer gate pulse for each successive scan. Finally, the array IY is averaged.

SUBROUTINE FILL simply accumulates data in the array IY and increments the variables NCALL and IBEF. The latter is necessary so that the RTS routine can start with a value of 1 for the buffer event flag.

SUBROUTINE SAMPLE uses DATAKE to acquire data and calls SUBROUTINE GETBKG which retrieves the dark signal data from the disk and subtracts it from the current contents of IY. The listing for GETBKG contains several statements with a "D" in column one. These can be included in the compilation by using the /D switch when running the RT-11 FORTRAN compiler. These statements call the display routines in the LPS supporting software so that the effects of subtracting the background from the averaged signal can be observed on the digital oscilloscope. The output of the sample and hold amplifier is negative, as is the output of the CCD. In order to display positive-going peaks the contents of IY are subtracted from the data in file BKG.DAT. This is done merely for the sake of convenience in subsequent display and plotting routines.

PROGRAM DIODE next calls SUBROUTINE SHOW which uses the display routines to display the contents of the buffer on the digital oscilloscope. This subroutine also has facilities for isolating portions of the nominally 1728-word buffer and displaying a smaller number of points so that detail can be 
investigated. If the number of points displayed is less than or equal to 30 , SUBROUTINE PARSE is called and values of the displayed points are typed on the terminal.

The final routine called in the program is SUBROUTINE DATRIT. This routine creates a file on the RKO5 disk which contains the current contents of the array IY, which is the element-by-element average of the results of LIM successive scans. The file can then be accessed by analysis programs at a future time.

A11 subroutines which write and read data to and from the disk use subroutines in the DEC System Subroutine Library SYSLIB. Thus, the subroutines PUTBKG, GETBKG, and DATRIT use SYSLIB elements to convert file names in ASCII to the Radix -50 format required for the file allocation routines IENTER and LOOKUP. SYSLIB calls are also used for identifying the RT-11 channel number to use for data transfers, to write or read data to or from specified files, and close and free input/output channels for later use. The number of words specified in the IWRITW and IREADW routines are transferred in one operation, making this technique the most efficient and rapid method for writing and reading data from mass storage. The 1728 elements which result from sampling the entire CCD array are written to 7 blocks on the disk in one operation.

The RT-11 LINK procedure for program DIODE is as follows:

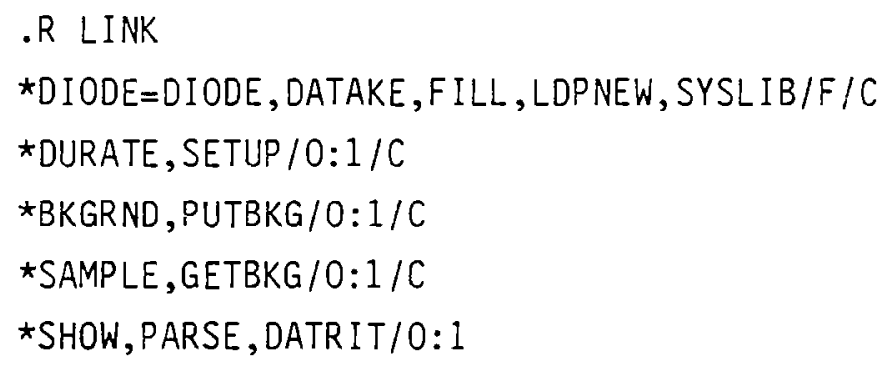

* Use of the overlay switch, 10:1, guarantees adequate room for operation of the program on a 16K-core machine, since only the subroutines necessary for operation are resident at any one time. Note that DATAKE and FILL are in the root segment of the link since they are accessed by subroutines in the overlay region.

A listing of PROGRAM DIODE fOIlows. 


\section{Program DIODE}

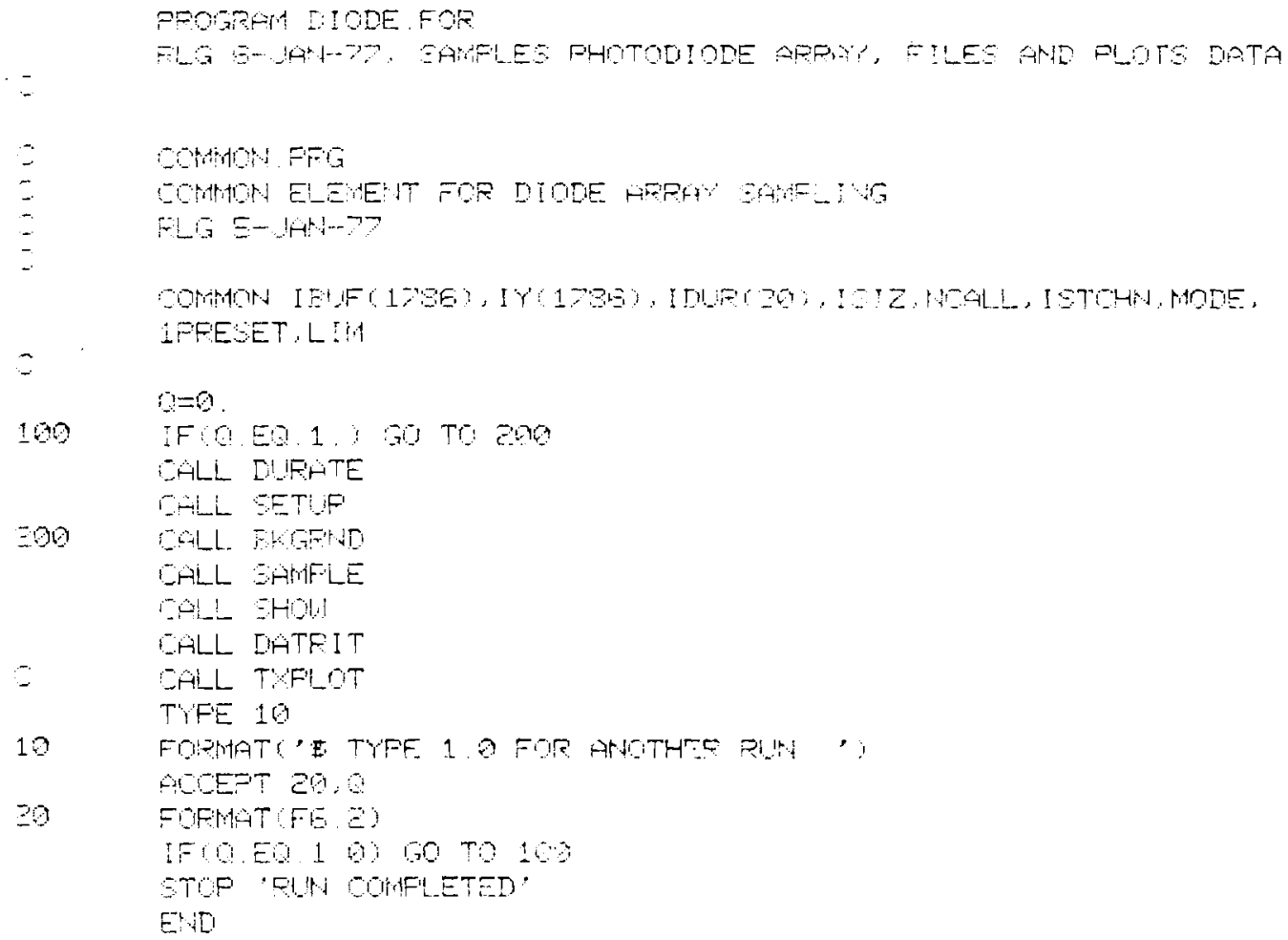




\section{SUBROUTINE DURATE}

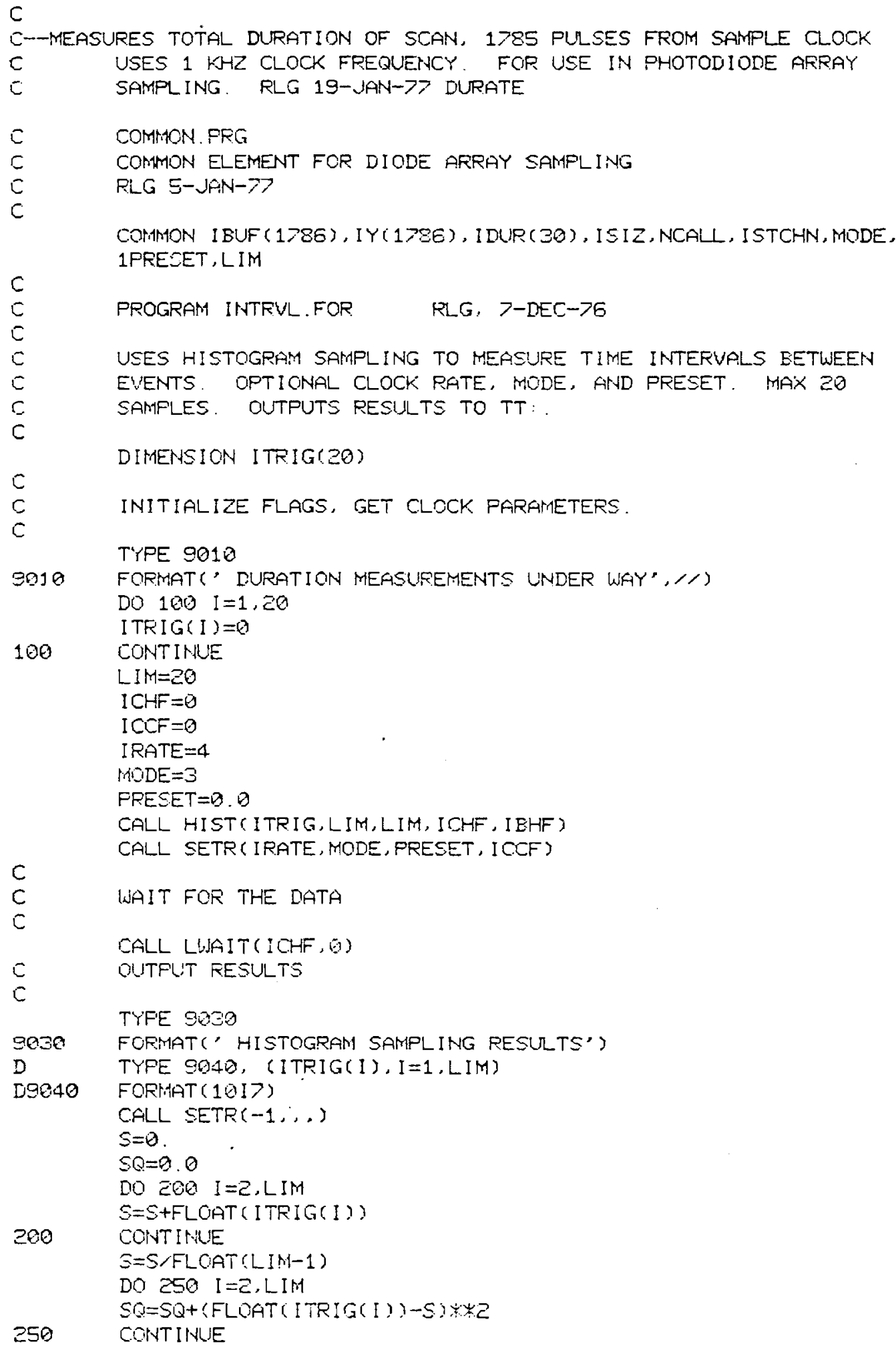


SUBROUTINE DURATE (contd)

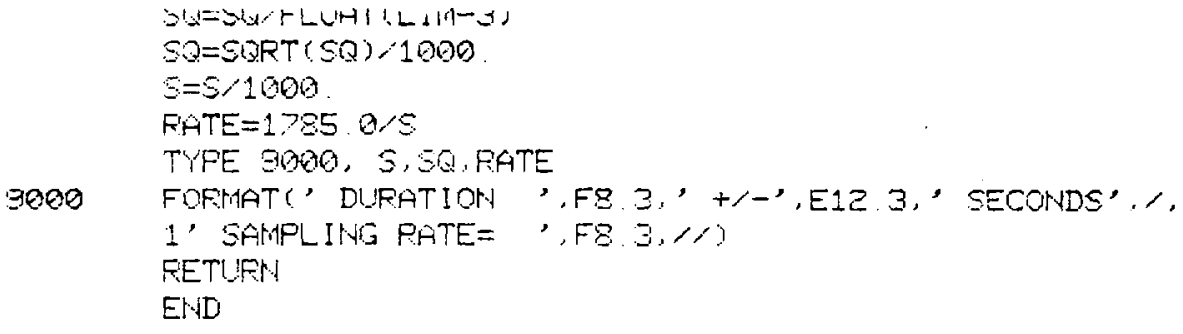

EVID 


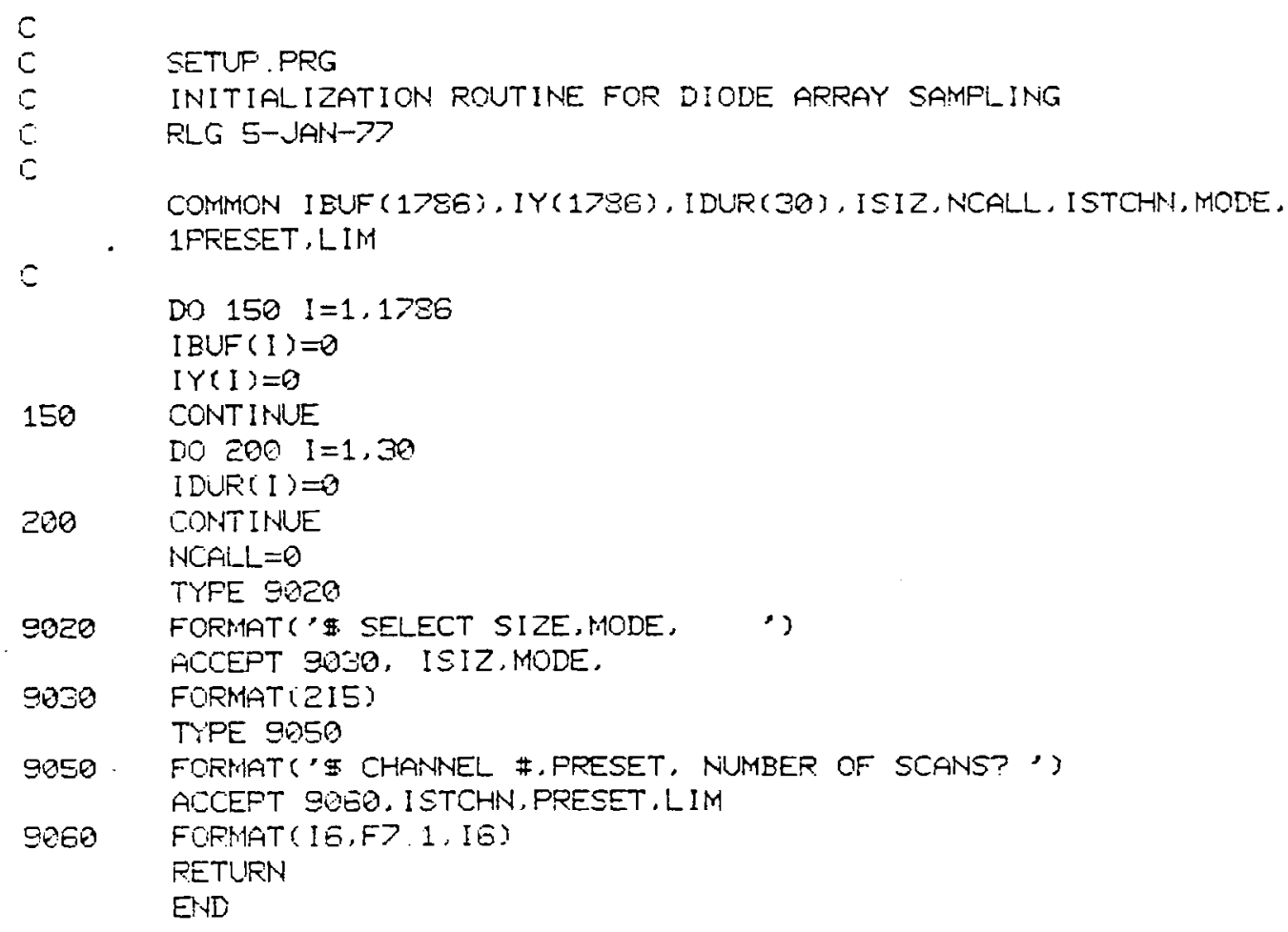

SUBROUTINE BKGRND

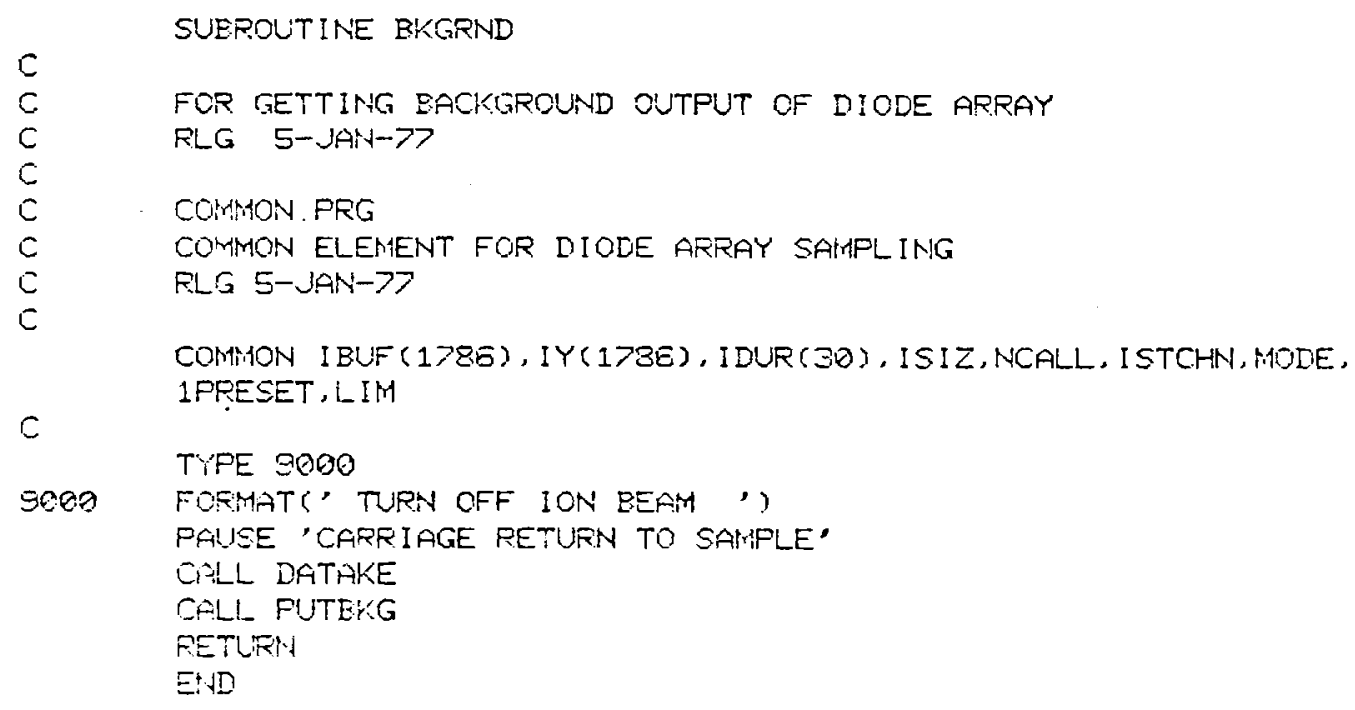




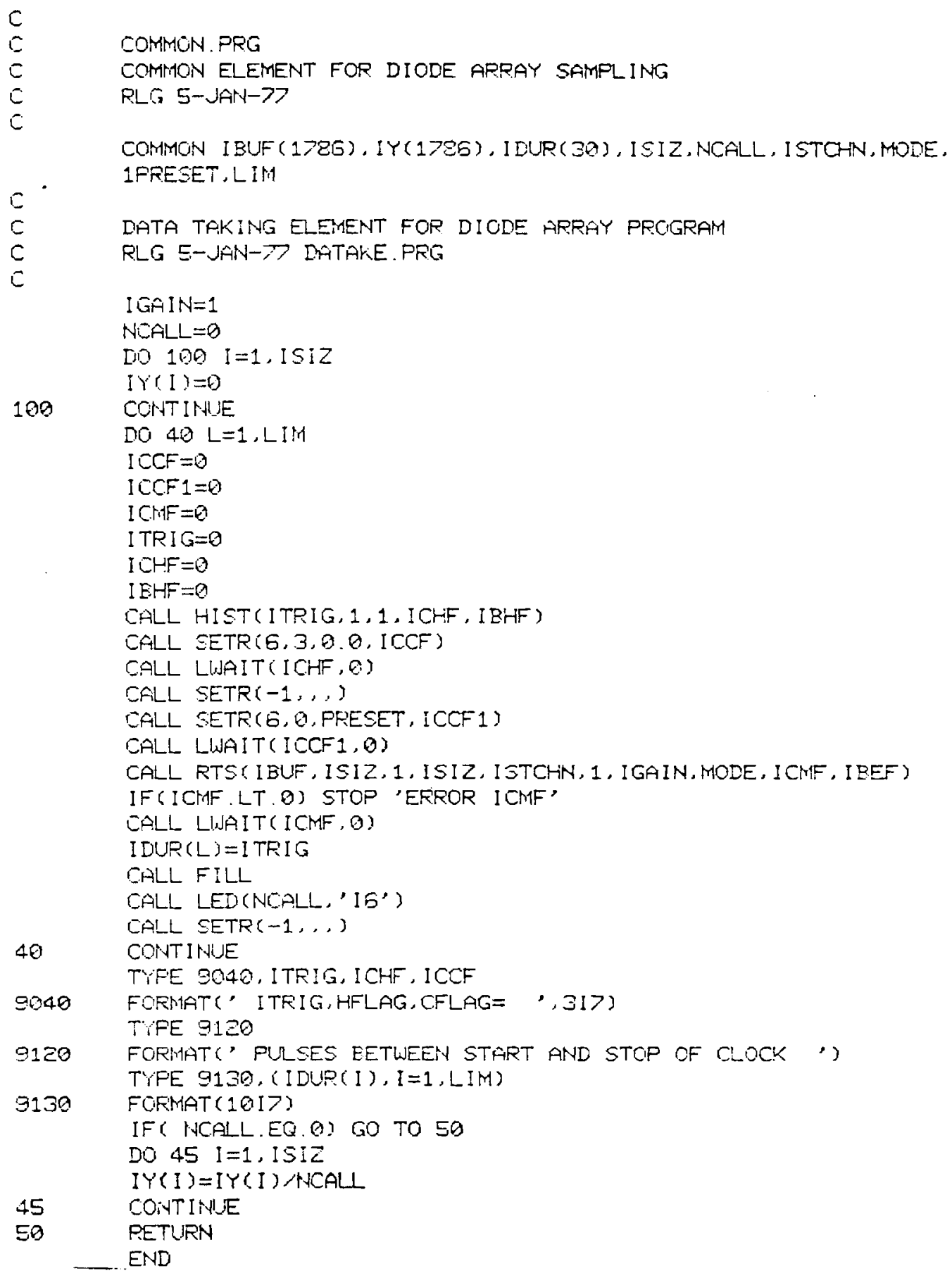


SUBROUTINE PUTBKG

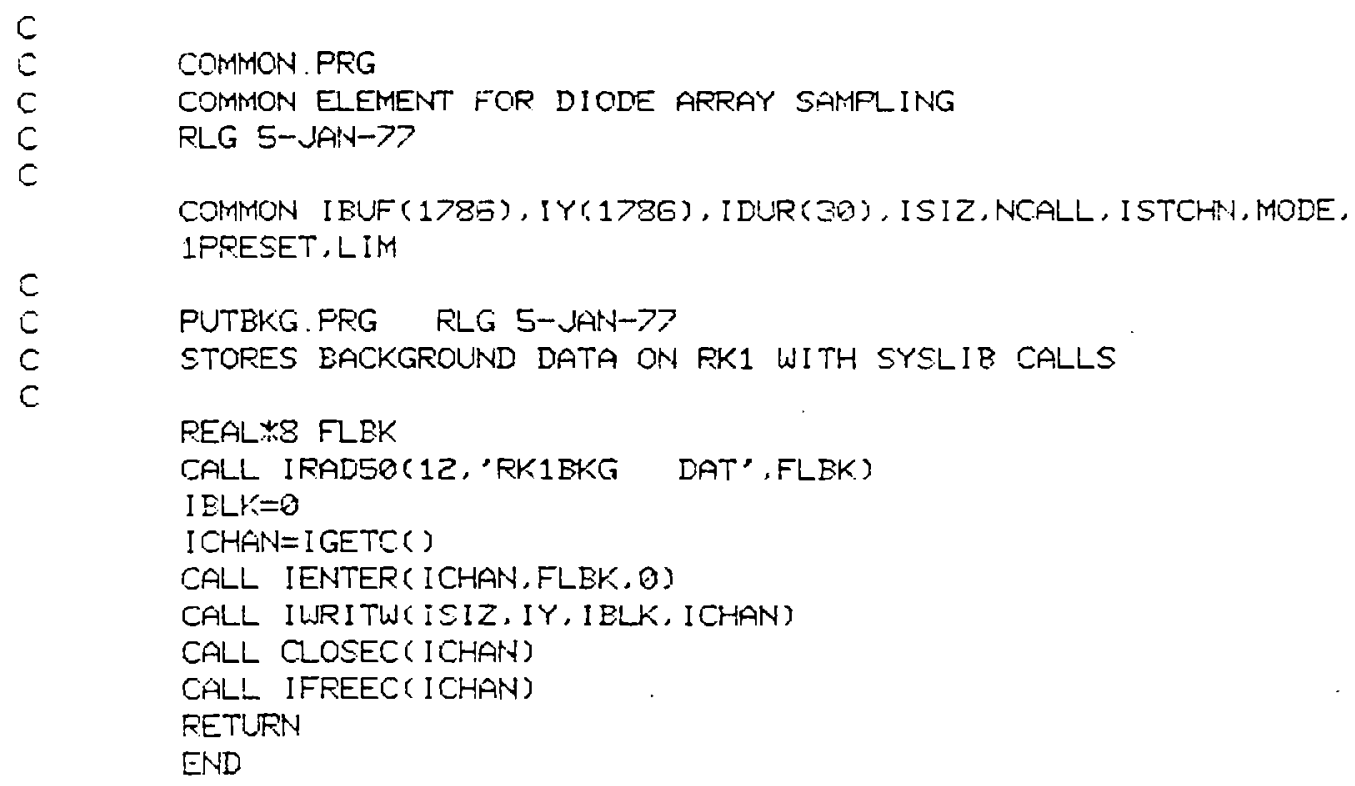

SUBROUTINE FILL

c

c

c

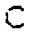

ADDS IEUF TO IY TO GET AVERAGE

COMHON IEUF (1786), IY(1>8E), IDUR( 20$)$, ISIZ, NCALL, ISTCHN, MODE, 1FRESET, LIM

10

DO $10 I=1, I S I Z$

$I Y(I)=I Y(I)+I E U F(I)$

CONTIRUE

NCALL $=$ NCALL+1

$I E E F=I B E F+1$

RETURT-

END 
SUBROUTINE SAMPLE

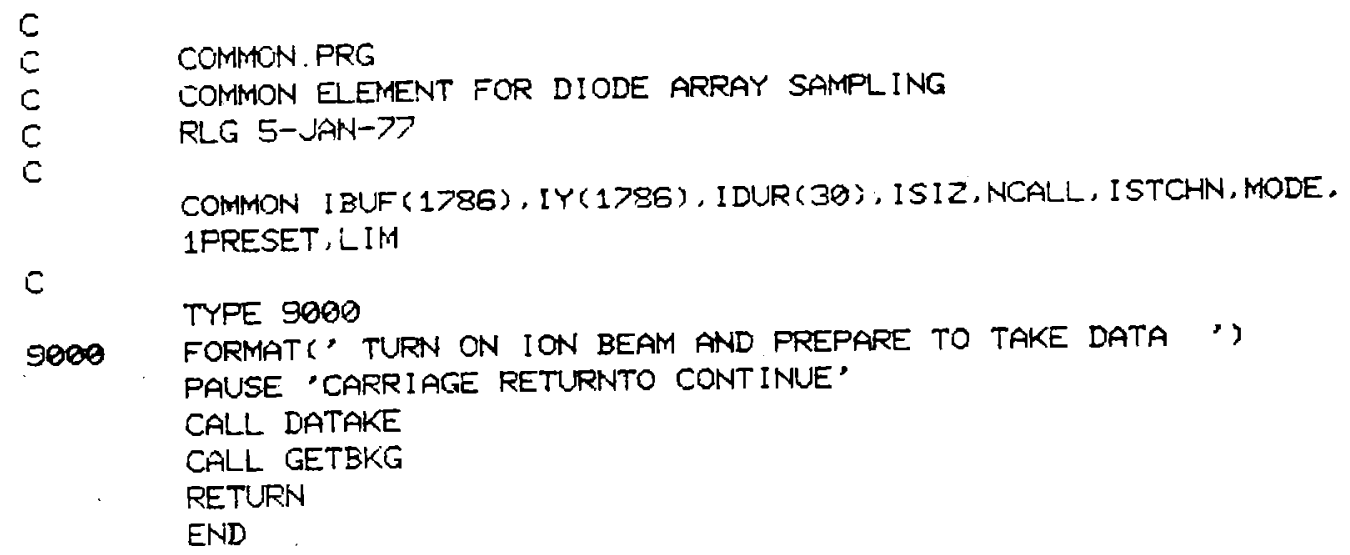

SUBROUTINE GETBKG

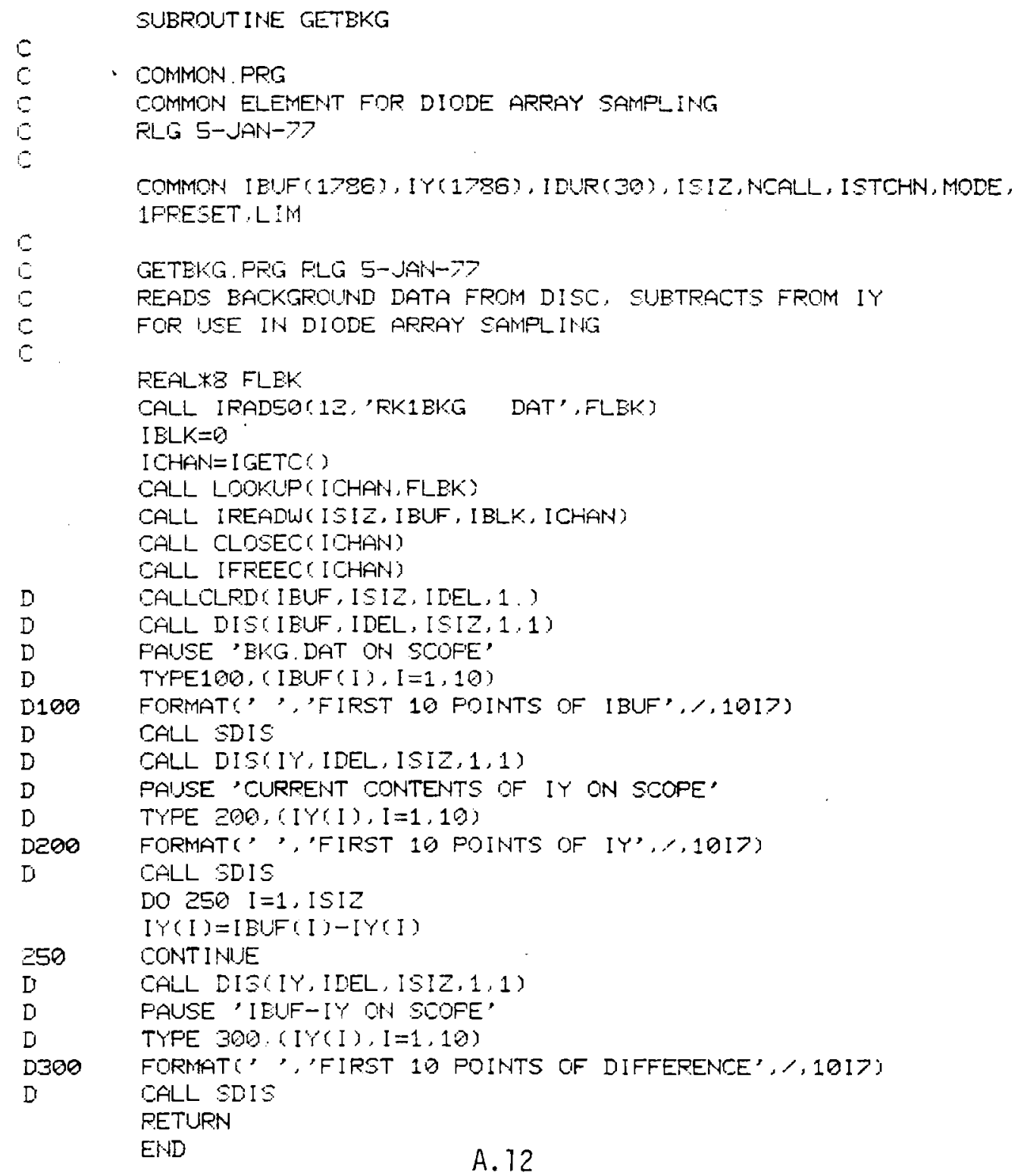




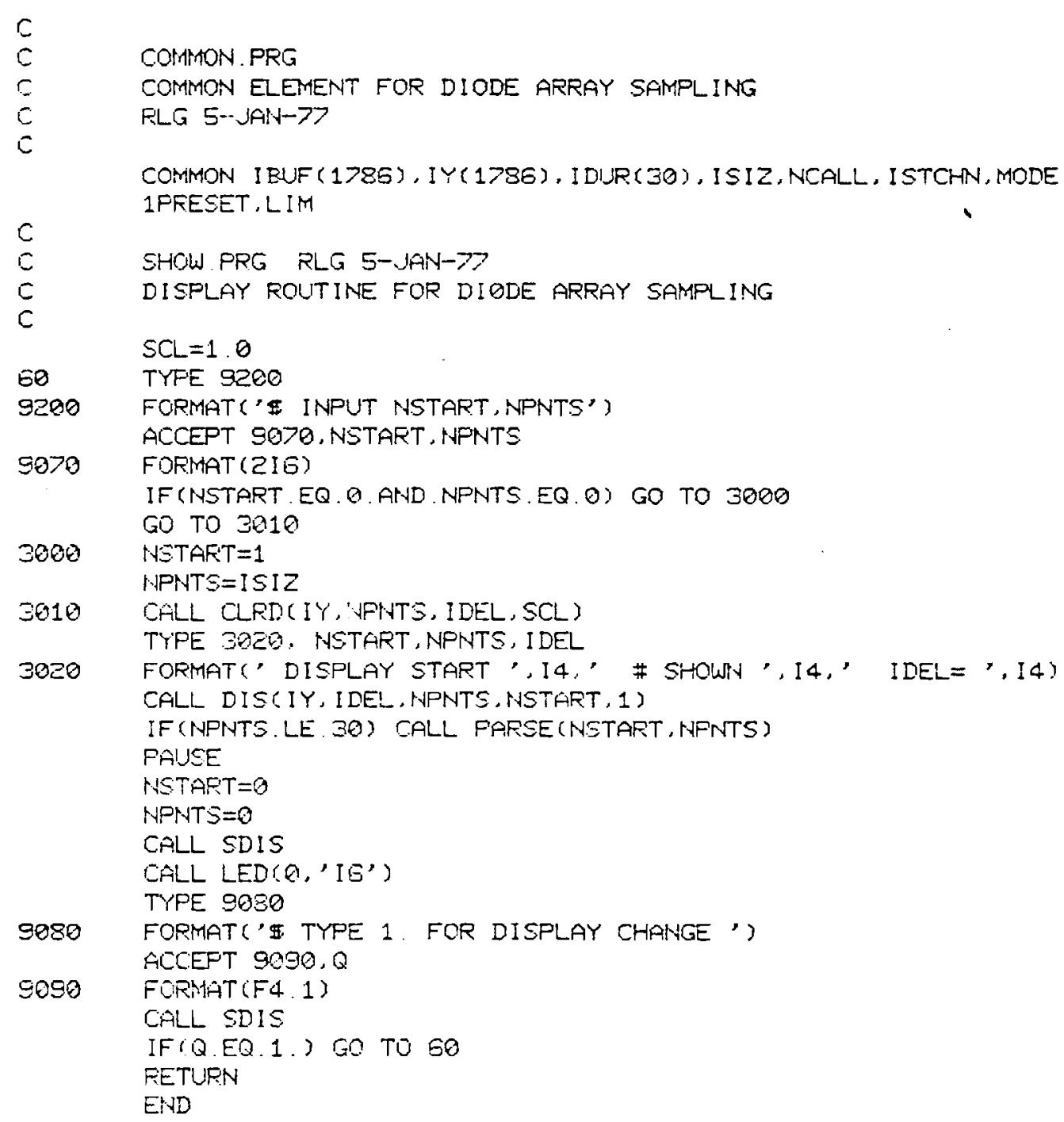


SLBROUTINE PARSE

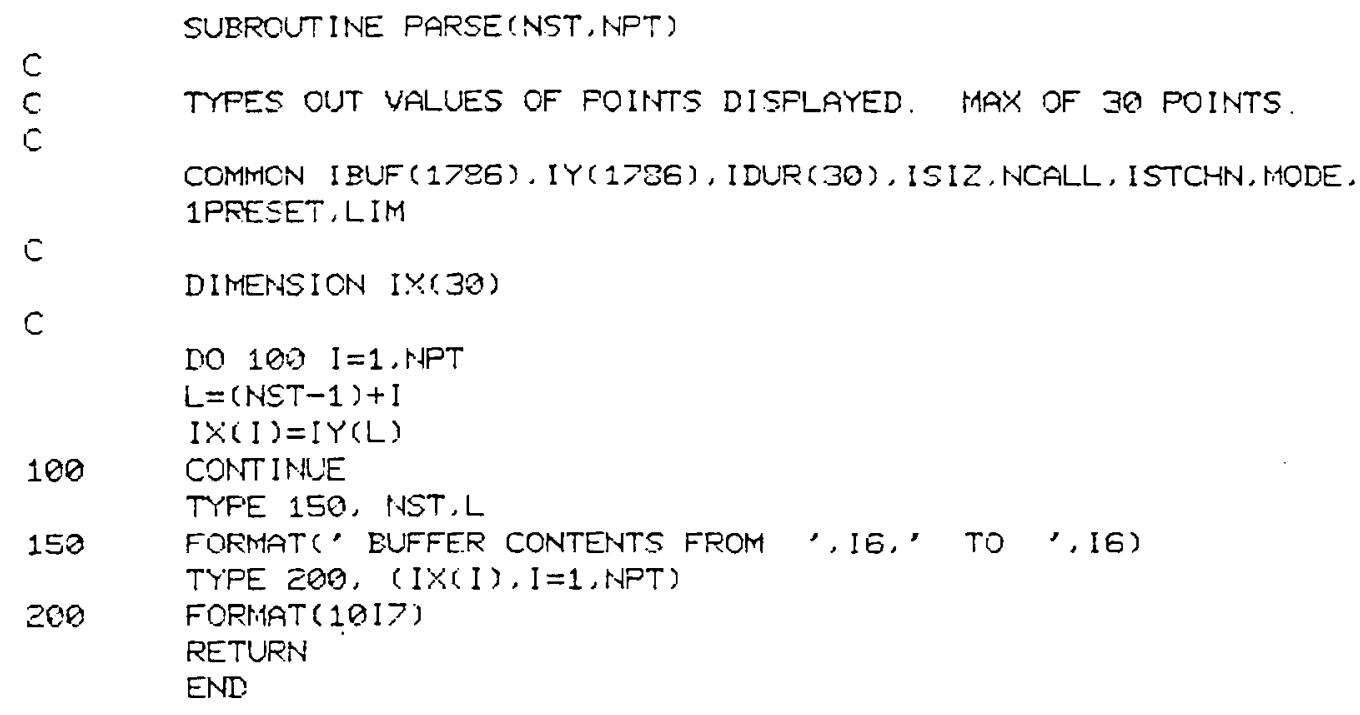

\section{SUBROUTINE DATRIT}

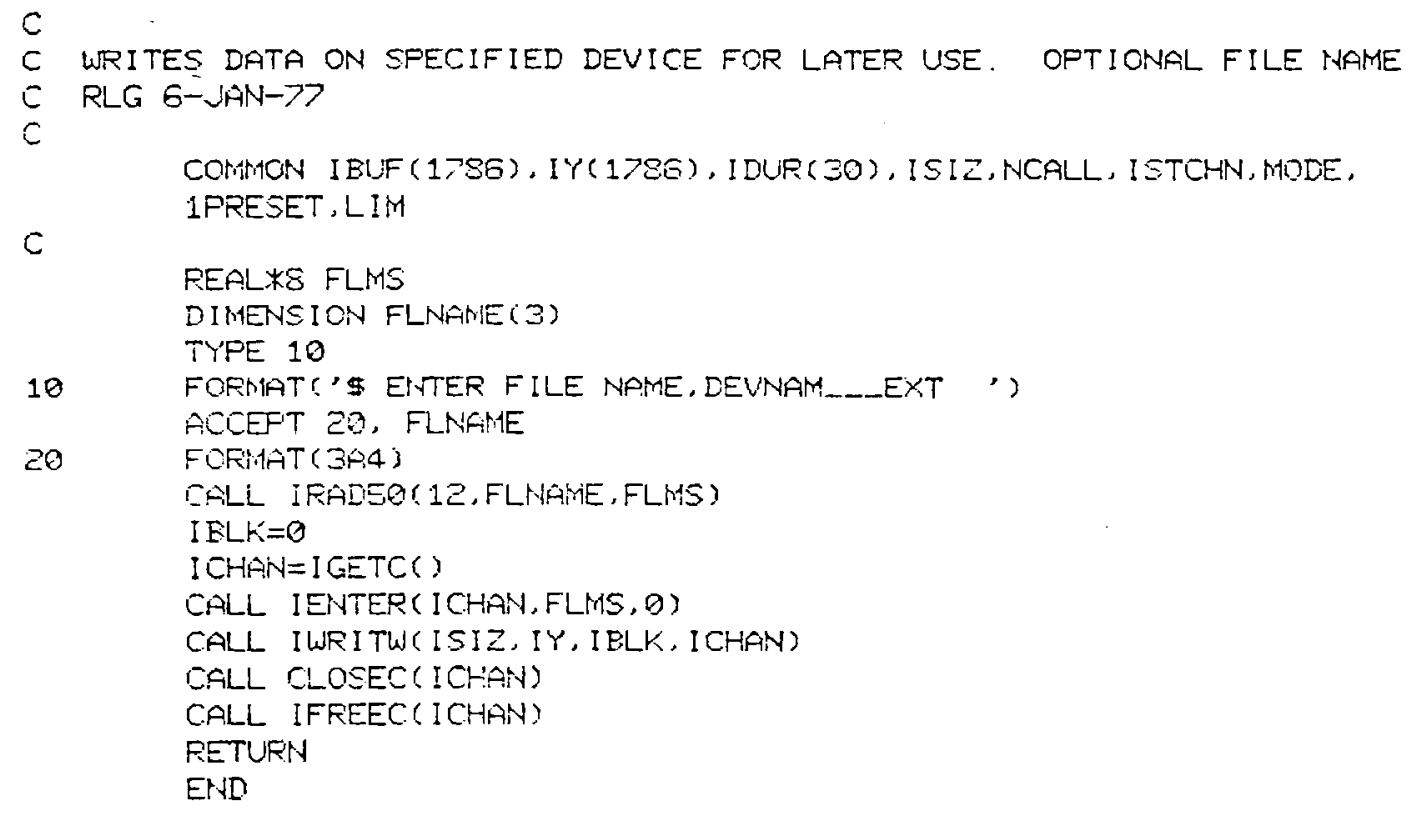


Program DIODE - Console Device Record

- h L L J OULE

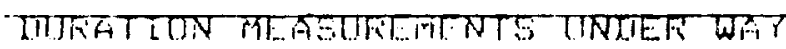

HISTOGFAM SAMFILING KESIJLTO.

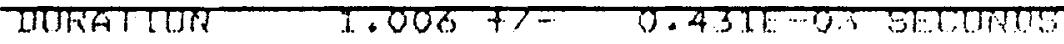

SAMFLING FIATE $=1774.725$

\begin{tabular}{|c|c|c|c|}
\hline जET:ECT & SLLE, MUIE, & 1728,0 & \\
\hline DFANMEI- & FYNTESET, & TUTPIJEER OS & SCARES \\
\hline
\end{tabular}

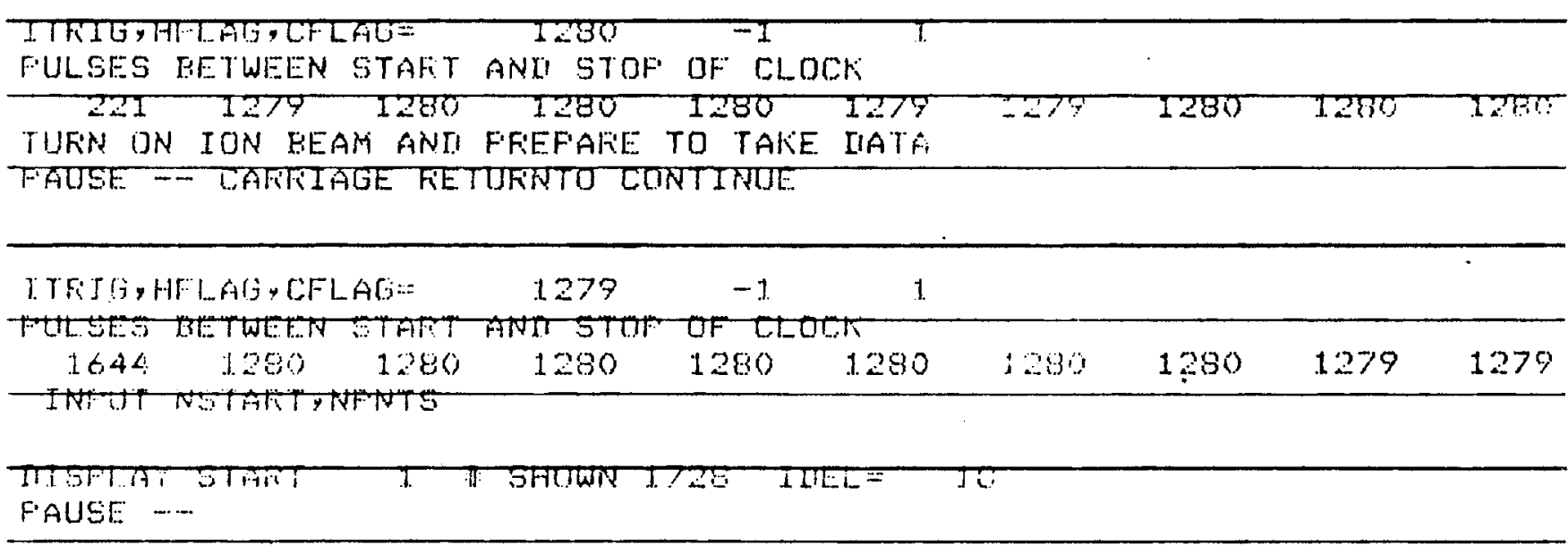

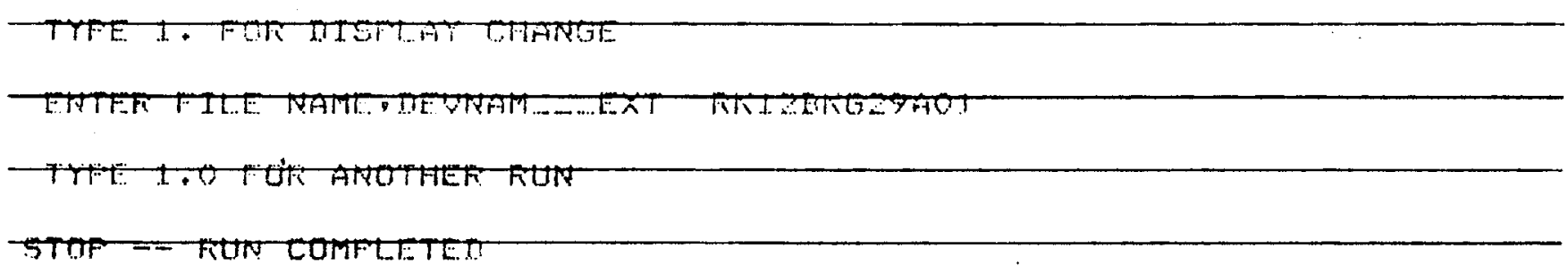




\section{A.2 PROGRAM PKREAD}

Program PKREAD retrieves data from the disk, draws a plot of the data on the Tektronix 4010 graphics terminal (TX), and uses the cross hairs on the terminal for reading the position of each side of a mass peak. These positions are then used as endpoints for finding the average maximum of each peak and performing a numerical integration between the limits defined by the endpoints. Computed values are then used to calculate abundance ratios and isotopic abundance of the recorded spectrum. Subroutine TXPLOT draws the plot of amplitude versus diode number on the Tektronix 4010. Subroutine AVMAX finds the maximum of each peak and calculates the average value of the maximum 5 points in the peak. Subroutine SUMIT performs the numerical integration using Simpson's one-third rule. Both AVMAX and SUMIT then calculate isotopic abundances and ratios and type results on the Console terminal.

TXPLOT is the first subroutine called by PKREAD. The routine first uses SYSLIB subroutines to fetch the TX handler and to identify and read the file to be plotted. The balance of the routine uses Device Independent Graphics (DIG) subroutines contained in the Battelle-Northwest library, BNWLIB.

After drawing and labeling the axes, a message from TXPLOT prompts the user to select the origin for the ordinate so that the data plot may be placed conveniently in the plotting field. After the plot has been drawn on the terminal the user is given the option of re-drawing the data at increased vertical expansion. This element was supplied to make extremely small peaks more visible on the plot and to permit better definition of the beginning and end points of the peak for cross-hair placement.

The next series of statements in PKREAD are required to read the positions of the cross-hair intersections of the Tektronix 4010 terminal. The FORTRAN direct read, established by the DEFINE FILE statement and the following READ statement, was found necessary for consistent reading of the crosshair positions. Following the TX read, the received values are corrected 
for offsets and scaling used in the plotting subroutine, TXPLOT, and pairs of limits are stored sequentially, the abscissa and ordinate value being stored in the arrays IDUR and IT, respectively.

Subsequent programming assumes that abscissa values for 1 imits increase monotonically, with the last upper limit read the largest. Meaningless results are produced if this condition is not satisfied. Limits for up to 15 mass peaks can be stored for analys is in subroutines AVMAX and SUMIT.

Subroutine AVMAX is a straightforward FORTRAN routine using standard techniques and functions for finding the maximum of each mass peak. Lower and upper limits for the DO loops in the individual searches through segments of the buffer IBUF are passed to AVMAX via the array IDUR. The first loop finds the maximum and the second identifies the index of the maximum value and stores it in the array MI. The average value of 5 points at the peak, including the maximum, is then computed and stored in array $Y$. Next, the standard deviation of the 5 points is determined and placed in array STO. After writing these results on the console terminal the routine finds the largest peak and computes and prints the ratios with respect to that peak. Abundance values are calculated and printed prior to the RETURN to the main program.

Subroutine SUMIT differs from AVMAX in that numerical integration over peak heights is used to determine isotopic ratios and abundances. The integration uses Simpson's rule in the form:

$$
\begin{gathered}
f(x) d x=(h / 3) f_{0}+4\left(f_{1}+f_{3}+\ldots+f_{n-1}\right)+ \\
2\left(f_{2}+f_{4}+\ldots+f_{n-2}\right)+f_{n},
\end{gathered}
$$

where the notation $f\left(x_{i}\right)=f_{i}$ has been used. The uniform interval has the value unity. The integral over the base line is calculated in closed form and stored in the variable BINT. After integrating is completed, the integral with respect to the origin is stored in array $x$, the base line integral is 
subtracted and the result stored in array $B X$. The results of the integration are typed on the console, and calculation of ratios and abundances proceeds as in AVMAX.

In both routines the variable LUP is used to sense completion of initial operations. For example, in SUMIT, after the integration is completed the result is stored in array $X$ and LUP is incremented by 2 . This is compared to the last upper limit contained in IDUR, and if it exceeds that value, the integration results are typed and the remaining calculations performed. The index $L$ is incremented prior to the test and once more at the start of the test so that the variable LO will always correspond to the lower integration limit.

A listing of the program and an example of the output produced are shown in the following. 


\section{Program PKREAD}

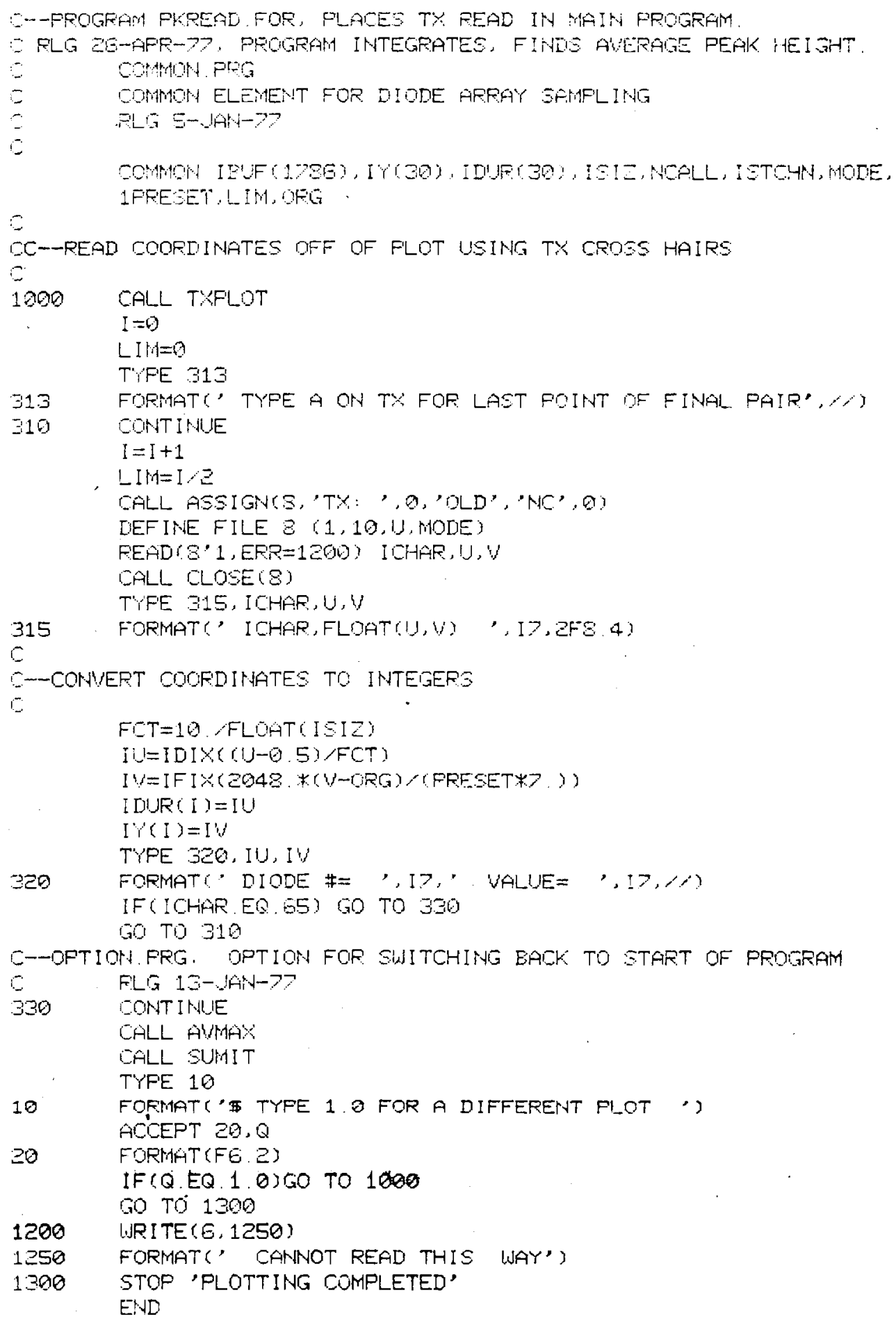


SUBROUTINE TXPLOT

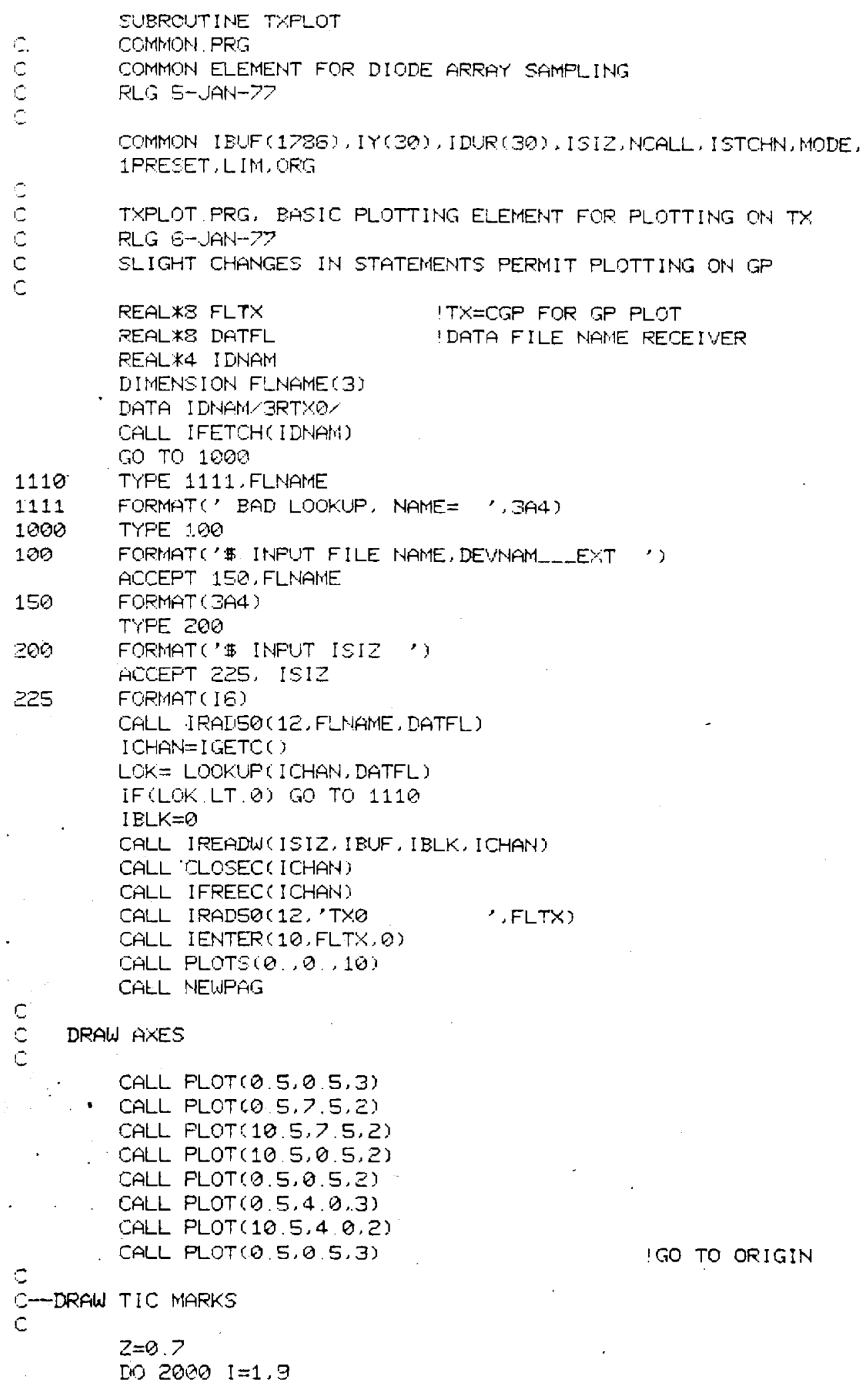


SUBROUTINE TXPLOT (contd)

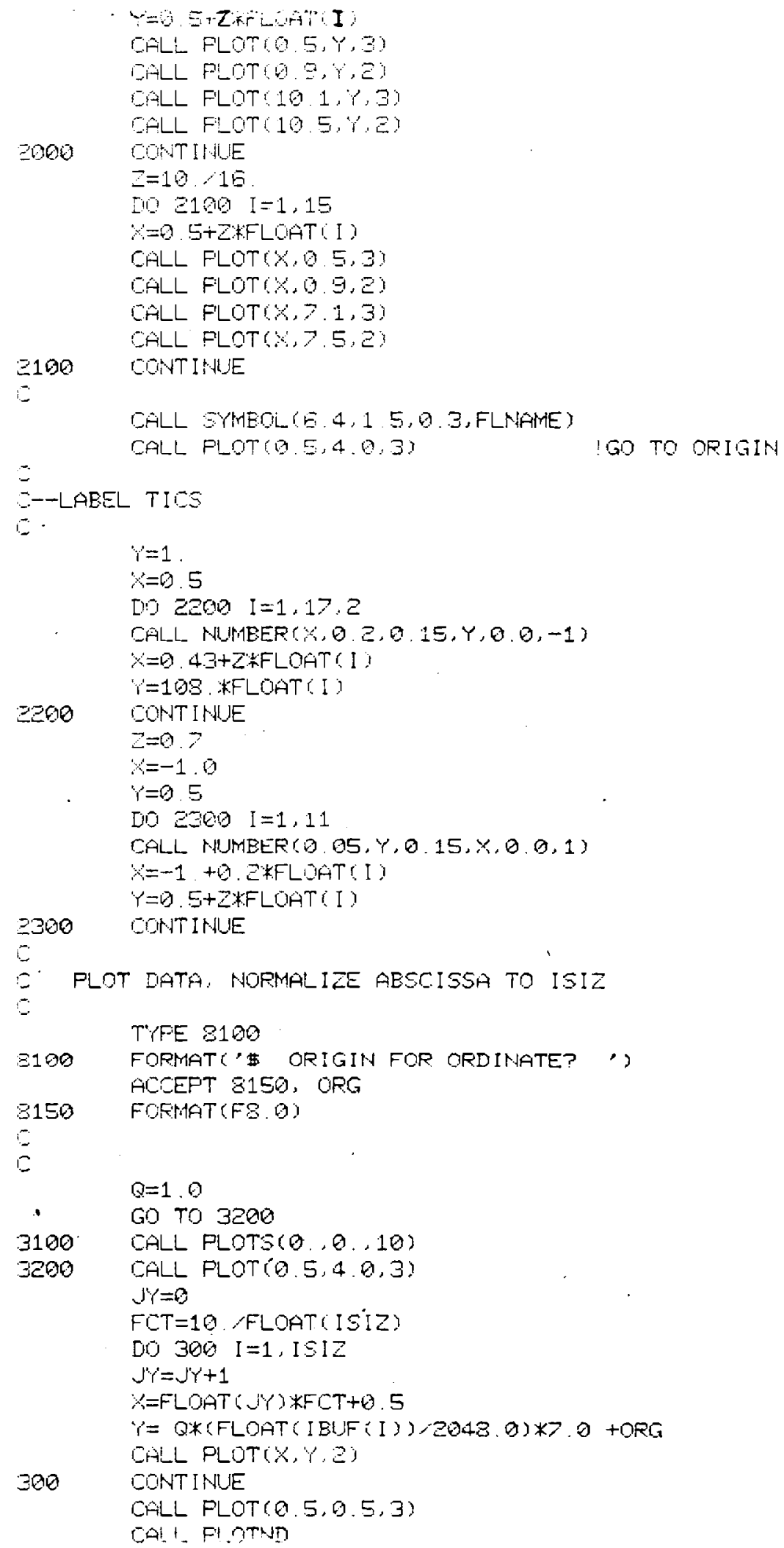




\section{SUBROUTINE TXPLOT (contd)}

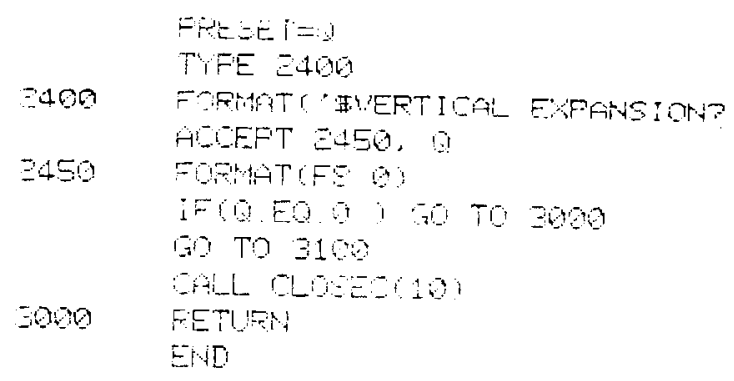




\section{SUBROUTINE AVMAX}

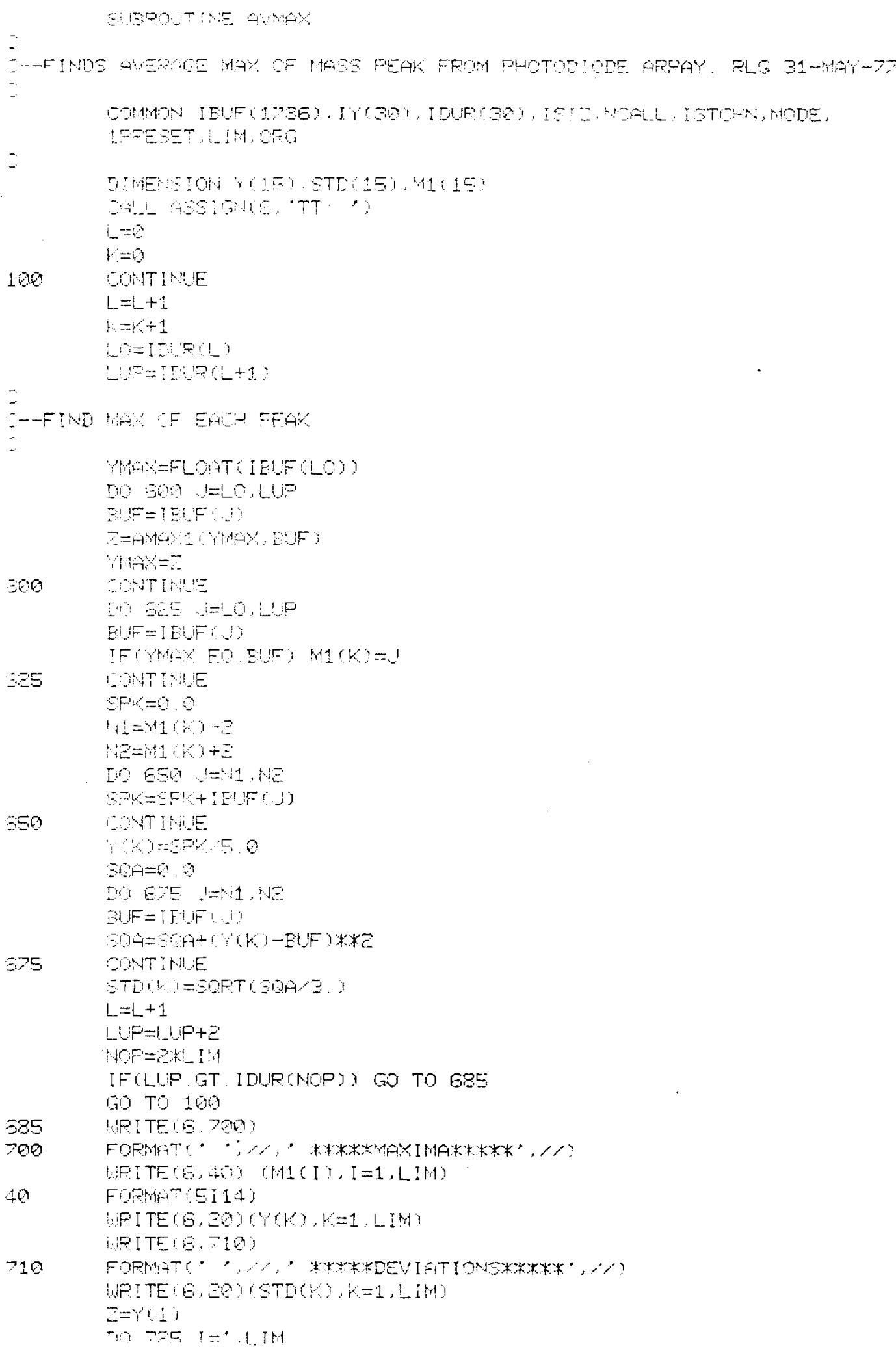


SUBROUTINE AVMAX (contd)

1 . .M. M- $2=-1+10 x$

725

T) 750 i=1, LIH

I I I I I I Y Y P

OTIHE

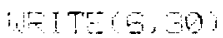

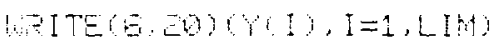

$\Delta \mathrm{UM}=0$

[10 $75 \quad I=1, L i+1$

$2 M A=9 M+Y(1)$

775 CONTIHUE

[10 $800 \quad i=1$, Lim

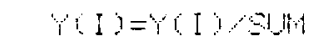

OTTINE

WITE ITE SED)

AEITEY EOYUI I I LIM

20 FOFHATSTA 5

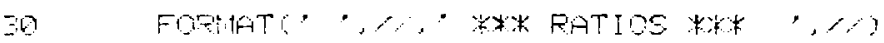

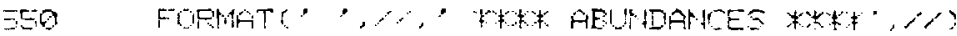

CHLL GLOEEOB)

FETURH

E [ 


\section{SUBROUTINE SUMIT}

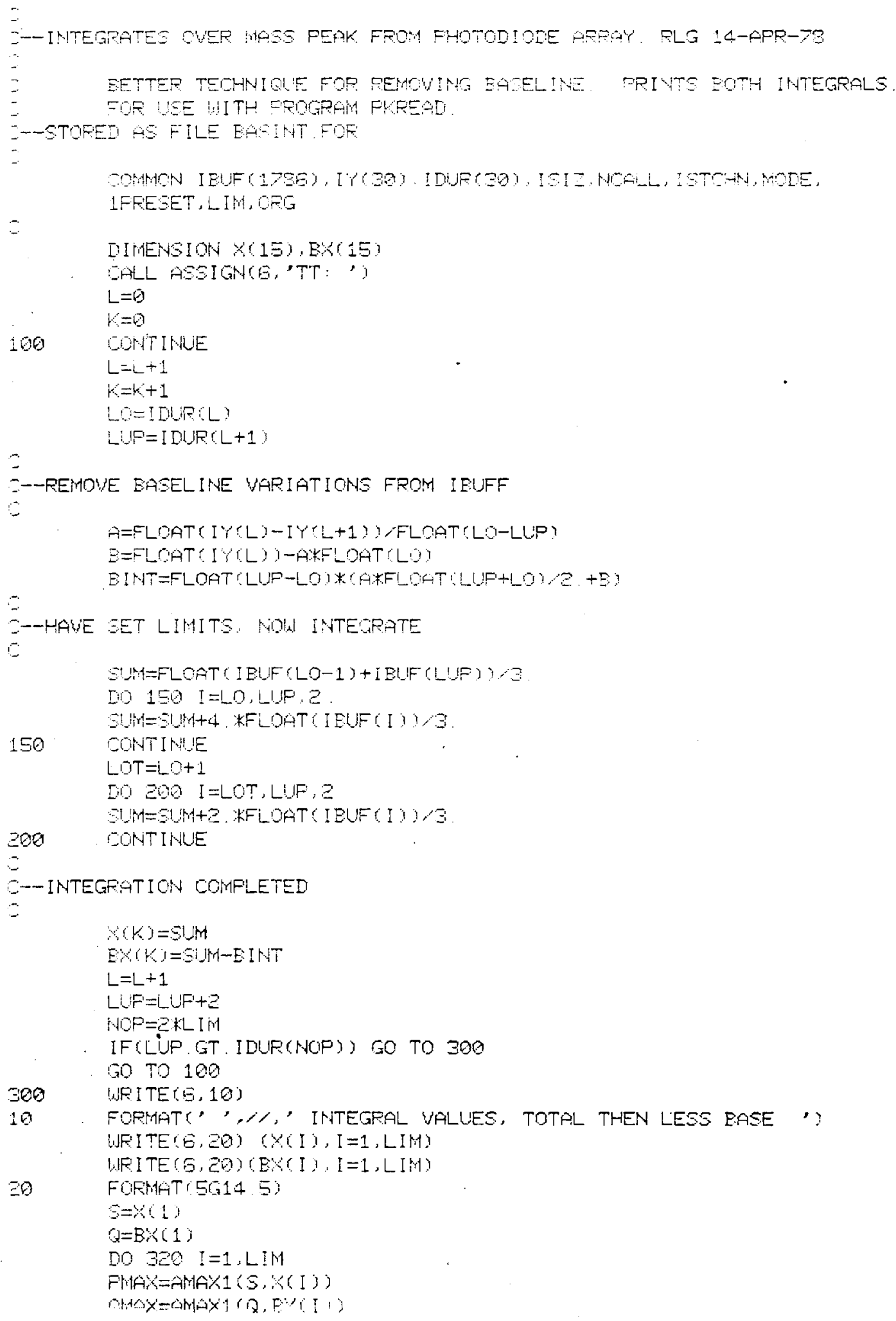


SUBROUTINE SUMIT (contd)

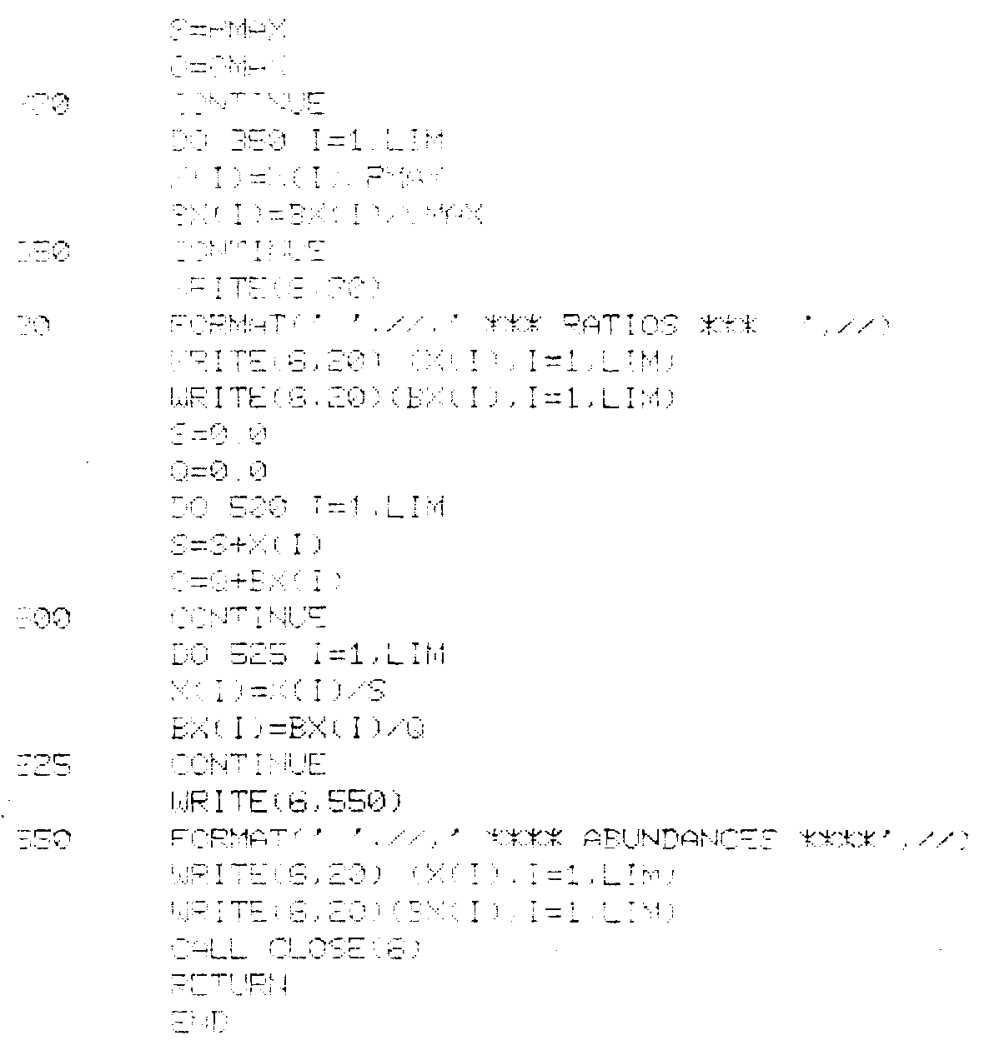


PKREAD Output

Some explanation of the output from program PKREAD is warranted. The first five messages come from TXPLOT and require user response. In this example file RG25A.003 from disk RKO was read. The file has 1728 words. The ordinate origin of 4.0 in. places the plotted data in the center of the plot field on the TX. After plotting the data as read, the data were re-plotted with twice the amplitude. A vertical expansion of 0 . caused return to the main program. Upon return to the main program, the cross hairs are displayed on the TX terminal and adjusted by the user. A read is performed when any key on the $T X$ is typed. Typing "A" terminates the reading session.

Two lines of output are produced for each read. The first line displays the variables ICHAR, $U$ and $V$. The latter two variables represent the coordinates of the cross-hair intersection from the origin of the TX. The variable ICHAR is the decimal equivalent of the ASCII octal code for the character typed. The next line of output contains the integer values corresponding to the coordinates $(U, V)$ adjusted for the origin of the plot axes. In the example shown, 5 peaks were read prior to analysis by AVMAX and SUMIT.

Results of analyses follow the record of the TX read. The first line of output displays the buffer position at which a maximum occurred and this is followed by the average maximum value. The balance of the output is self exp lanatory. 


\section{Program PKREAD - TX Read Record}

FE⿵冂卄

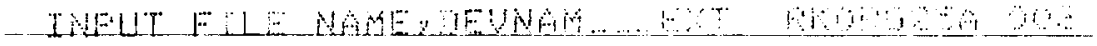

TNFUT TSTZ 1728

OFEOTH FOF OFOTHAE:

YERTICAL EXFANSTON? O

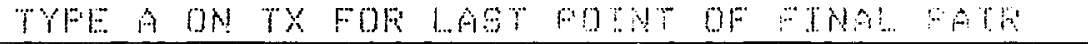

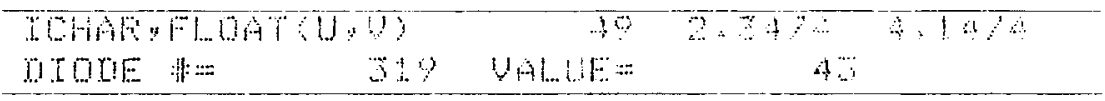

\begin{tabular}{|c|c|c|c|}
\hline TCHAR, FLOAICU:O & 4 & 3.0100 & $4.156 \mathrm{~B}$ \\
\hline DUOCE :AF: & $4+\ldots 11:=$ & 40 & \\
\hline THANE & 50 & $4,1 \% 9 \%$ & 4.1150 \\
\hline 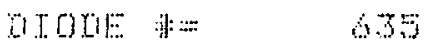 & UALUE: & 33 & \\
\hline
\end{tabular}

\begin{tabular}{|c|c|c|c|}
\hline 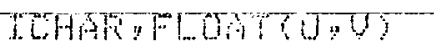 & 50 & $1,0,106$ & $4+116$ \\
\hline II] OTIE :H:=: & GALUE= & $3 y$ & \\
\hline$(1090)$ & $3 i$ & 6.916 & $\Leftrightarrow, 1 \%$ \\
\hline DTOTIE \#非 & $\forall A L U E=$ & $A \dot{A}$ & \\
\hline
\end{tabular}

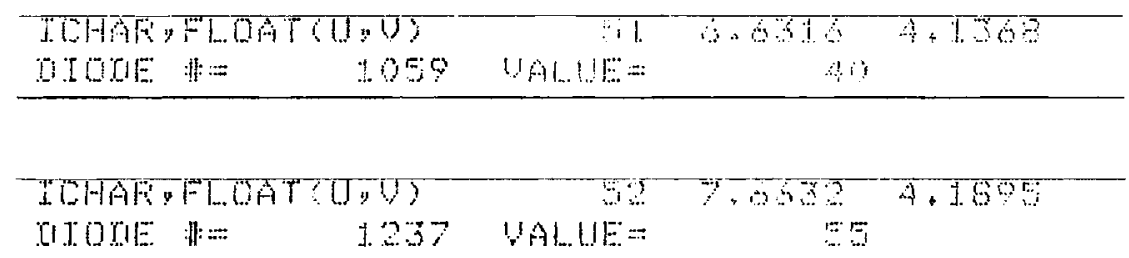

\begin{tabular}{|c|c|c|c|}
\hline TCHAR "TOATCU, & 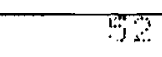 & $8 \cdot 36 \overline{6}$ & 4.144 \\
\hline OLORE \#:= & UALLUE: & 48 & \\
\hline TCHAR :FLOAT:UYU & 53 & $9+2.11$ & 4,056 \\
\hline DTOME :H:= & YALLEE= & 15 & \\
\hline 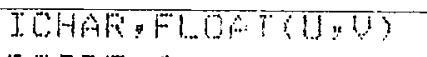 & 6 & क्ष & $4+0421$ \\
\hline OUOTIE & YHLAEN & 12 & \\
\hline
\end{tabular}




\section{Program - TX Read Record (contd)}

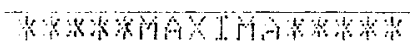

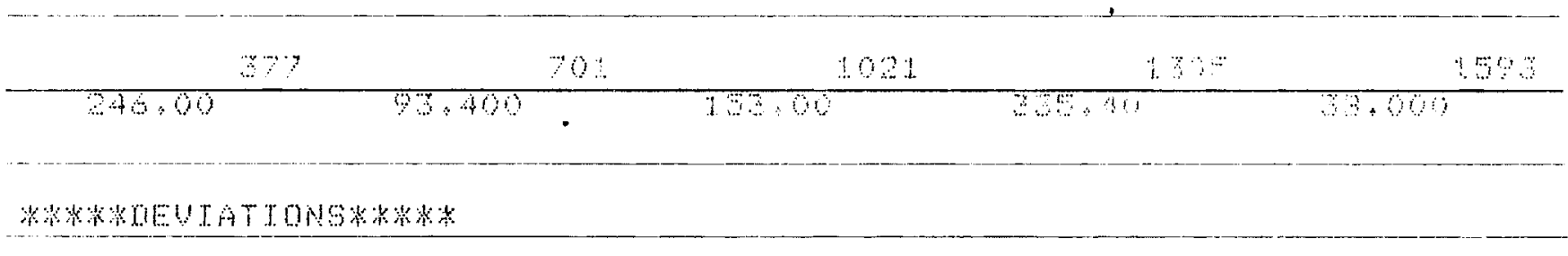
$+490 x$
$3+3+67$
$3 \times 7+17$
$3+9016$
$2+495$

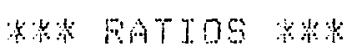

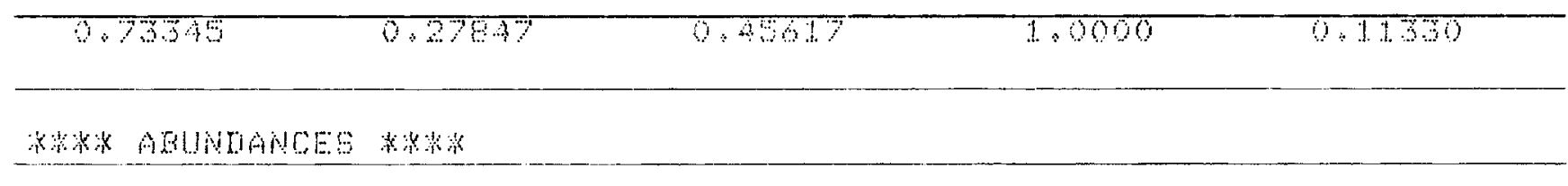

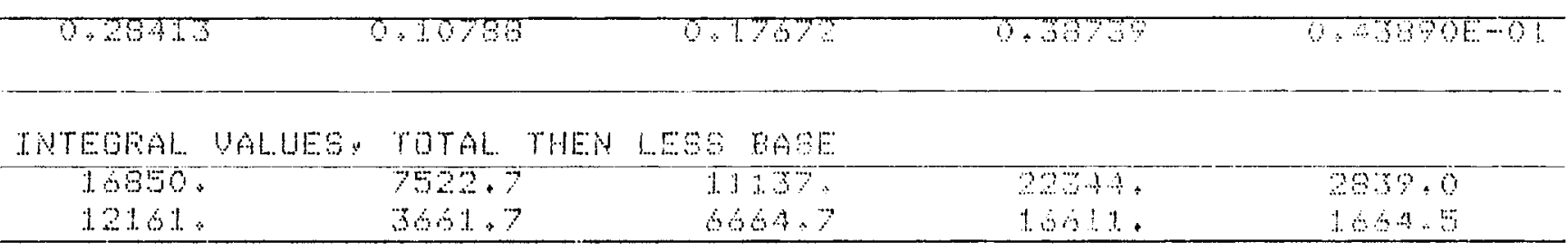

XXस WATIOB WR

\begin{tabular}{|c|c|c|c|c|}
\hline 0.7512 & 0.33067 & $0+47642$ & 1.0000 & 9.12706 \\
\hline $0+73206$ & 0.20044 & 0.40122 & .1 .0000 & $0+2.0020$ \\
\hline 决决次 ADUNIANCES & 乘乘乐 & & & \\
\hline 0.27763 & $0+1.395$ & 1.18 .49 & 0.363 .5 & $0.267 / 7 \mathrm{~m}$ \\
\hline 0.29833 & $0.89830 F-01$ & $0.1 .6,00$ & 0.40751 & $0.4063 \mathrm{al}-01$ \\
\hline
\end{tabular}

TIFE T.O FOF A DTFFERENT MOT

$-3 T O P=-$ FIOTIING ETMFLETEII 


\section{A.3 PROGRAM GLDPLT}

Program GLDPLT uses a Gould 5000 printer/plotter (GP) to produce hard copy of data taken by program DIODE. The file specified in FLNAME containing ISIZ words is read using SYSLIB routines. The program then writes a plot file, PLOTOO.DIG, on disk RKO, writes the contents of the input buffer IBUF on the GP in integer form, and finally chains to program DIDOT to translate the plot file on the disk to a hard copy plot on the GP. DIDOT then chains back to GLDPLT so that another file can be read and processed without returning to the monitor. To insure proper operation of the chain, the monitor command ".SET USR NO SWAP", must be issued prior to running GLDPLT.

Initialization for the program includes calls to IFETCH to load necessary device handlers. These calls are not required for program operation. They were inserted prior to learning that the SET USR NO SWAP command allowed correct operation of the chain procedure and were never removed. The same is true of the ASSIGN command which allocated channel 5 to the terminal.

The commands necessary to read the data are identical to those in TXPLOT except for the case when dark signal data are to be processed. The file containing that data is always named BKG.DAT and always contains values as recorded in program DIODE. Data are recorded on a $+/-1$ volt ADC with the value 2048 being produced for zero input voltage. The plots of background data would therefore be nearly full-scale on the paper since the output of the CCD under dark conditions is always a small negative voltage. Consequently, in case the file name has the suffix DAT, that file is subtracted from 2048 before writing and plotting. The other files are only scaled in the plotting routine since they were sent to the disk file as positive numbers by program DIODE.

The plotting routine in GLDPLT is similar to that in TXPLOT, the major difference being that the axes drawn on GP are rotated $90^{\circ}$ with respect to those on the TX. Both plotting routines draw a 9 in. $x 6.5$ in. frame for plotting. 
Data are written in 16 columns, labeled 1 through 16. Sampling of a full array therefore causes 108 lines of numerical output for the 1728 diodes in the array.

Program DIODOT is an element of the Pacific Northwest Laboratory (PNL) Device. Independent Graphics Package. This program converts the plot file written on the disk in floating point to dot mode and transfers the dot mode output to the electrostatic printer/plotter.

A listing of GLDPLT and an example of its output are shown in the following. In the example, two copies of the desired output are obtained. Only one is shown. 


\section{Program GLDPLT}

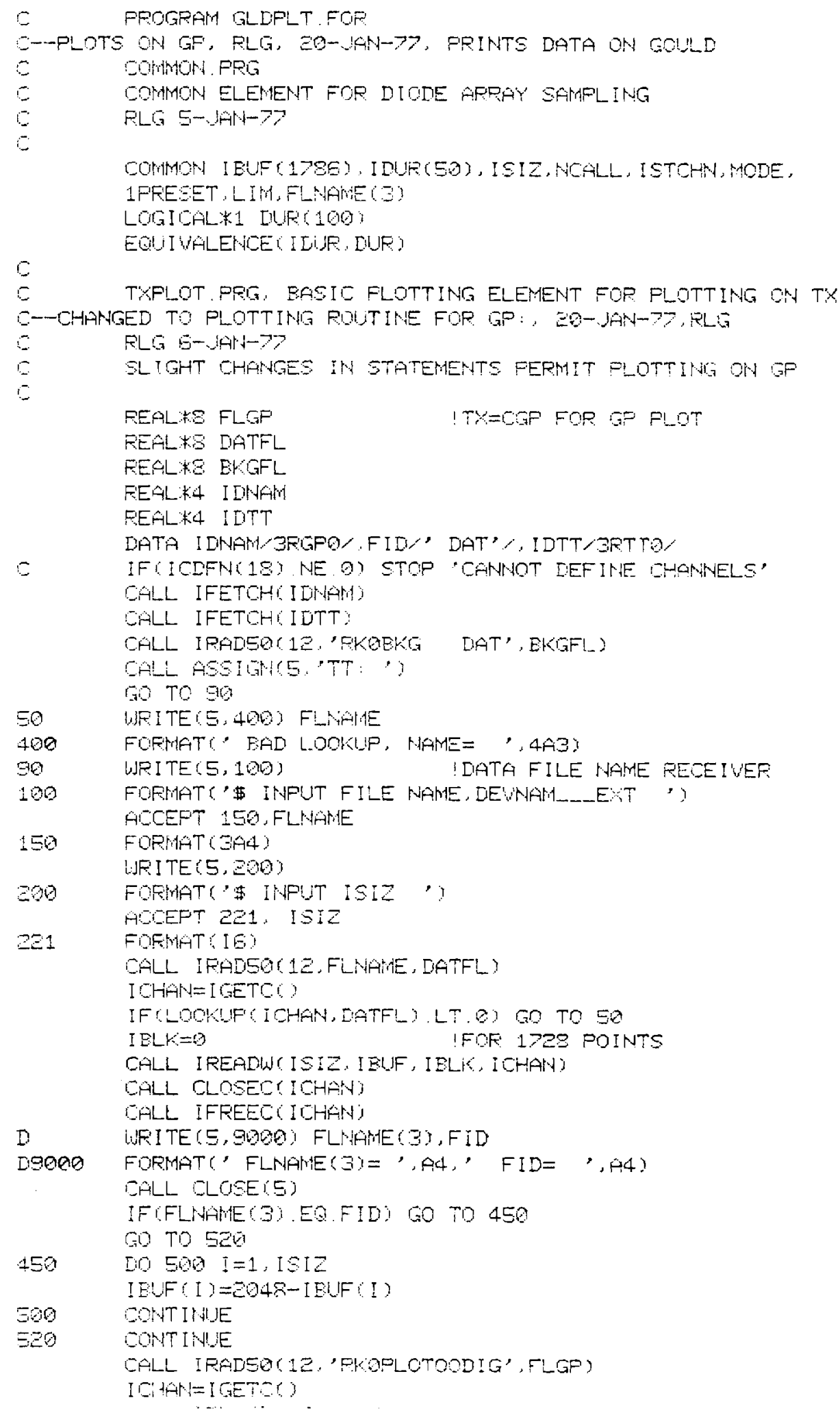


Program GLDPLT (contd)

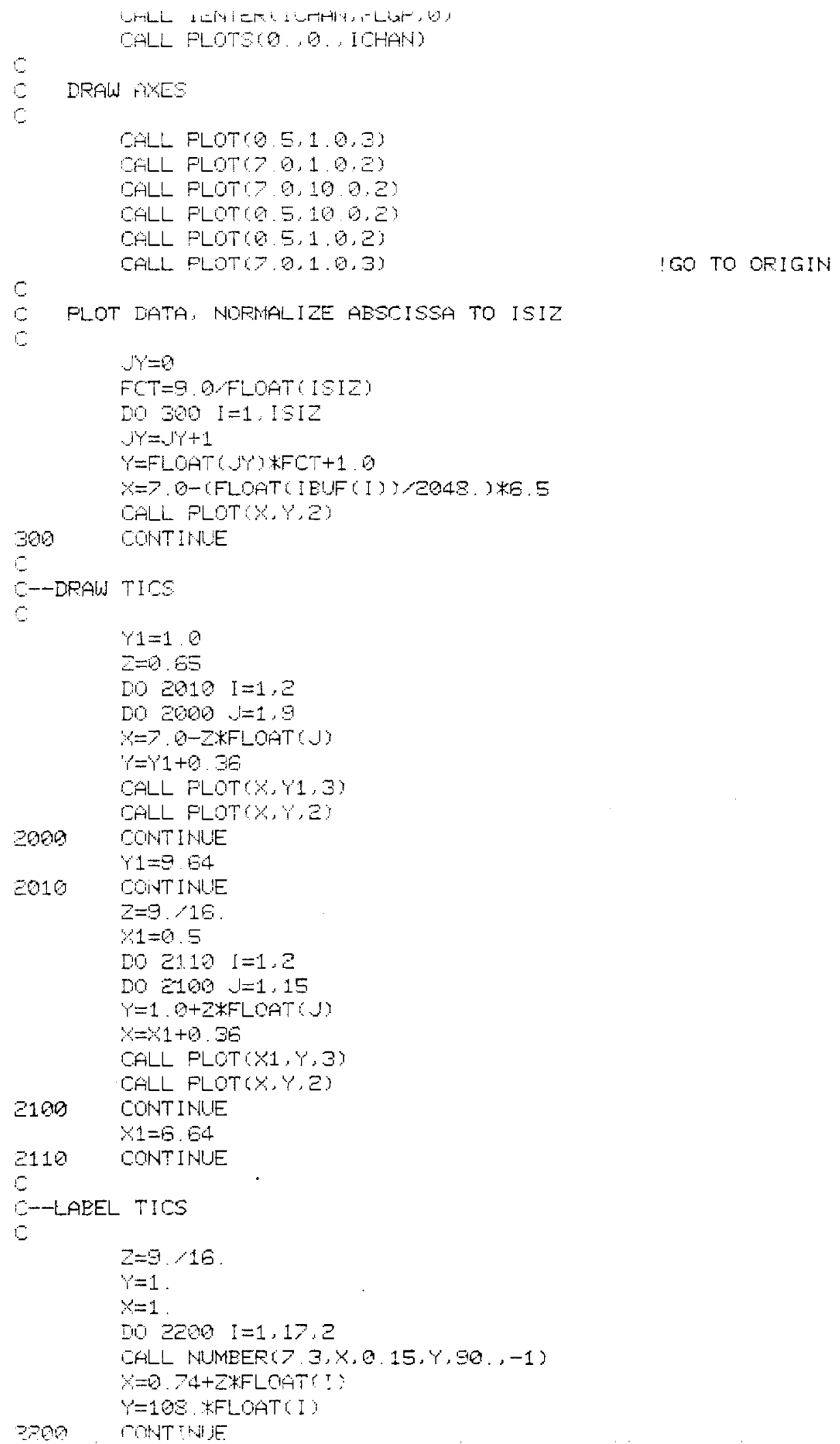




\section{Program GLDPLT (contd)}

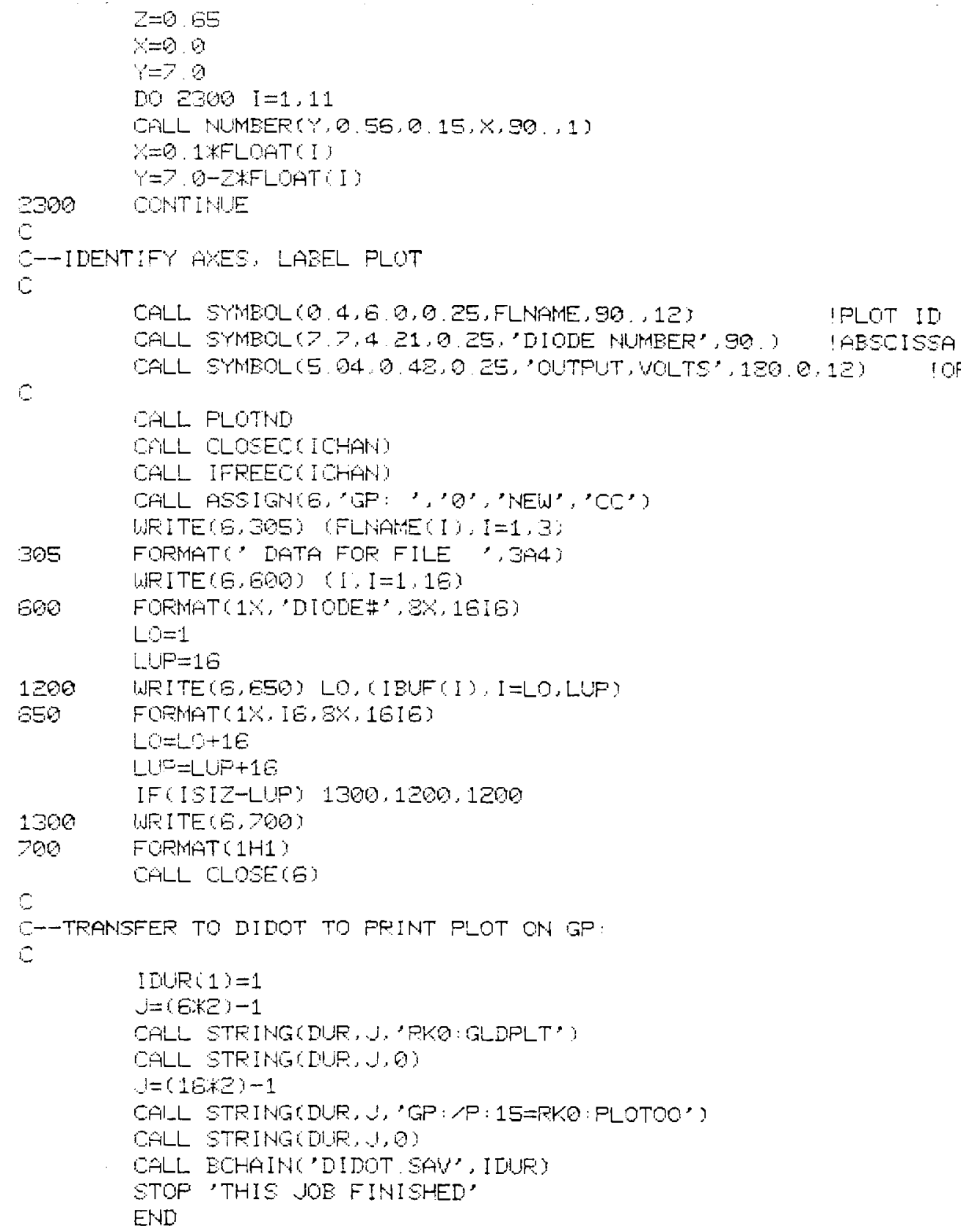




\section{Program GLDPLT - Read Record}

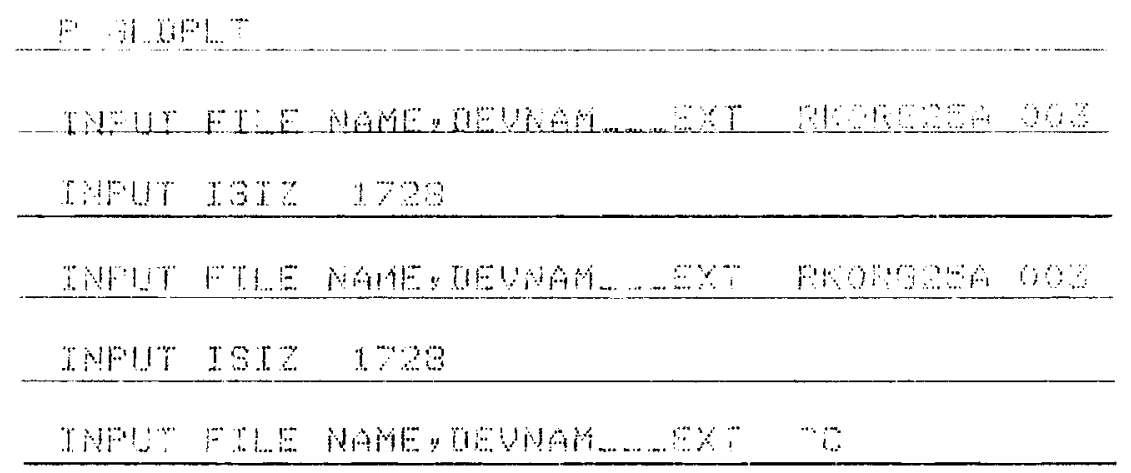




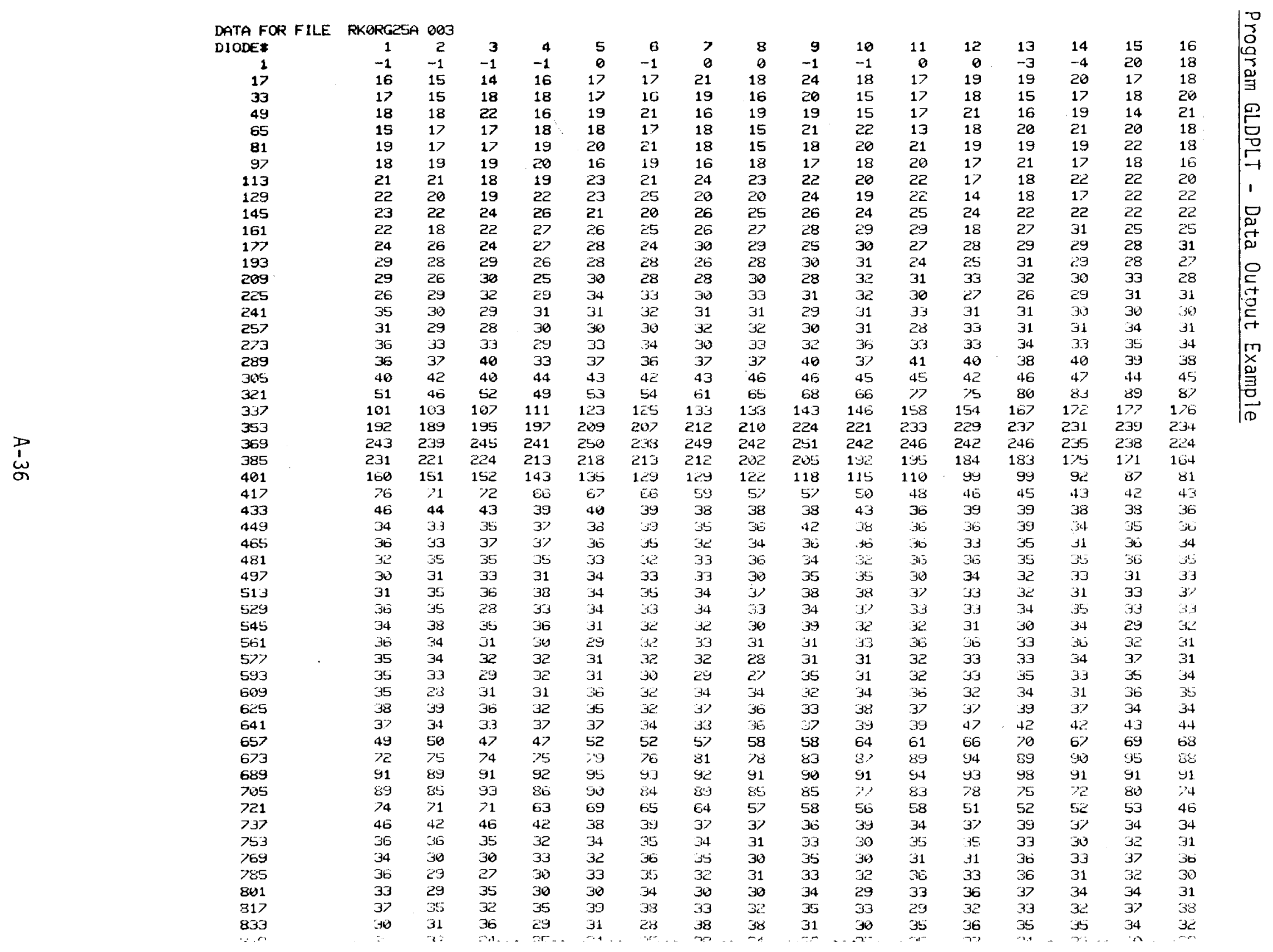




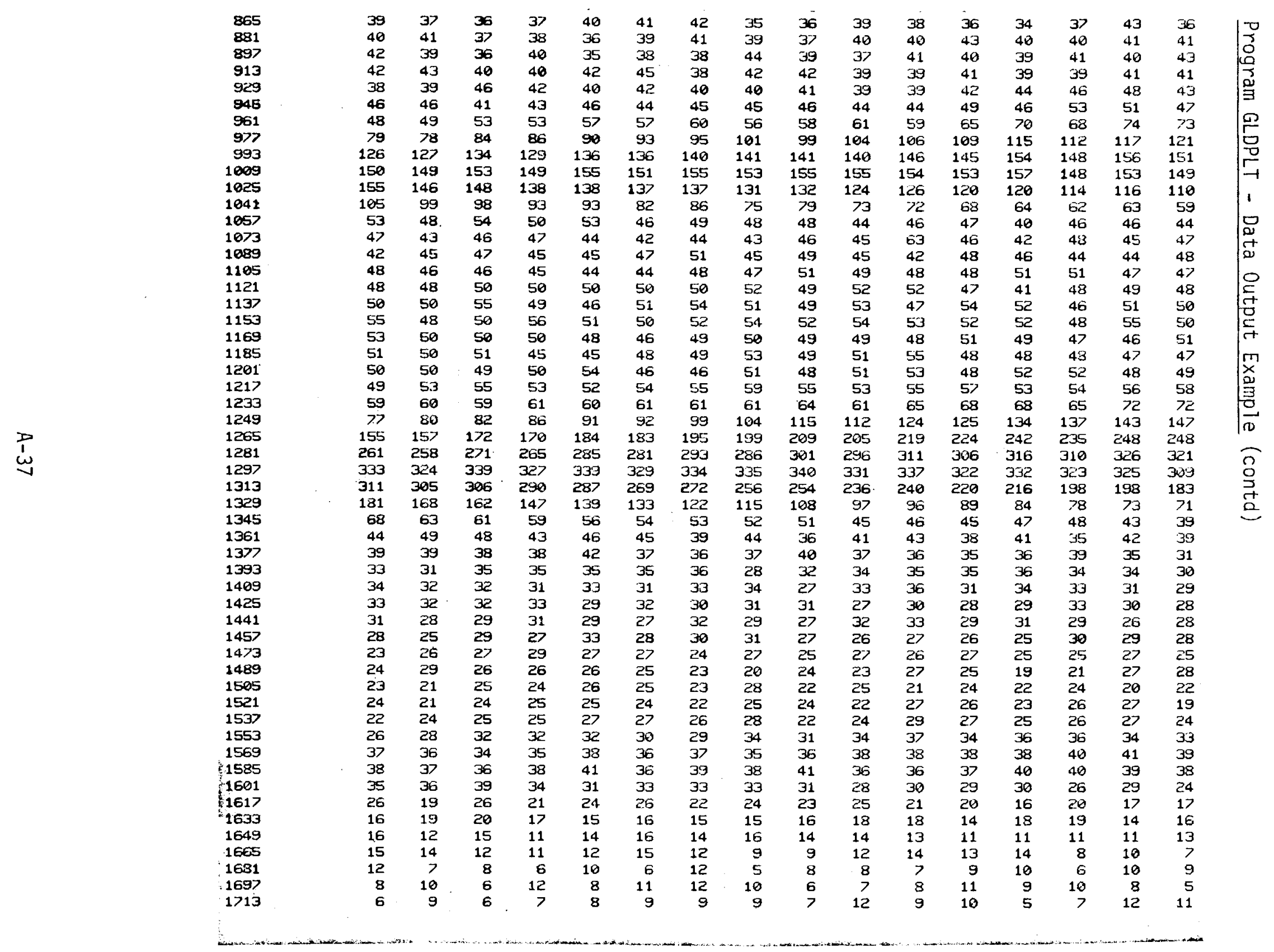




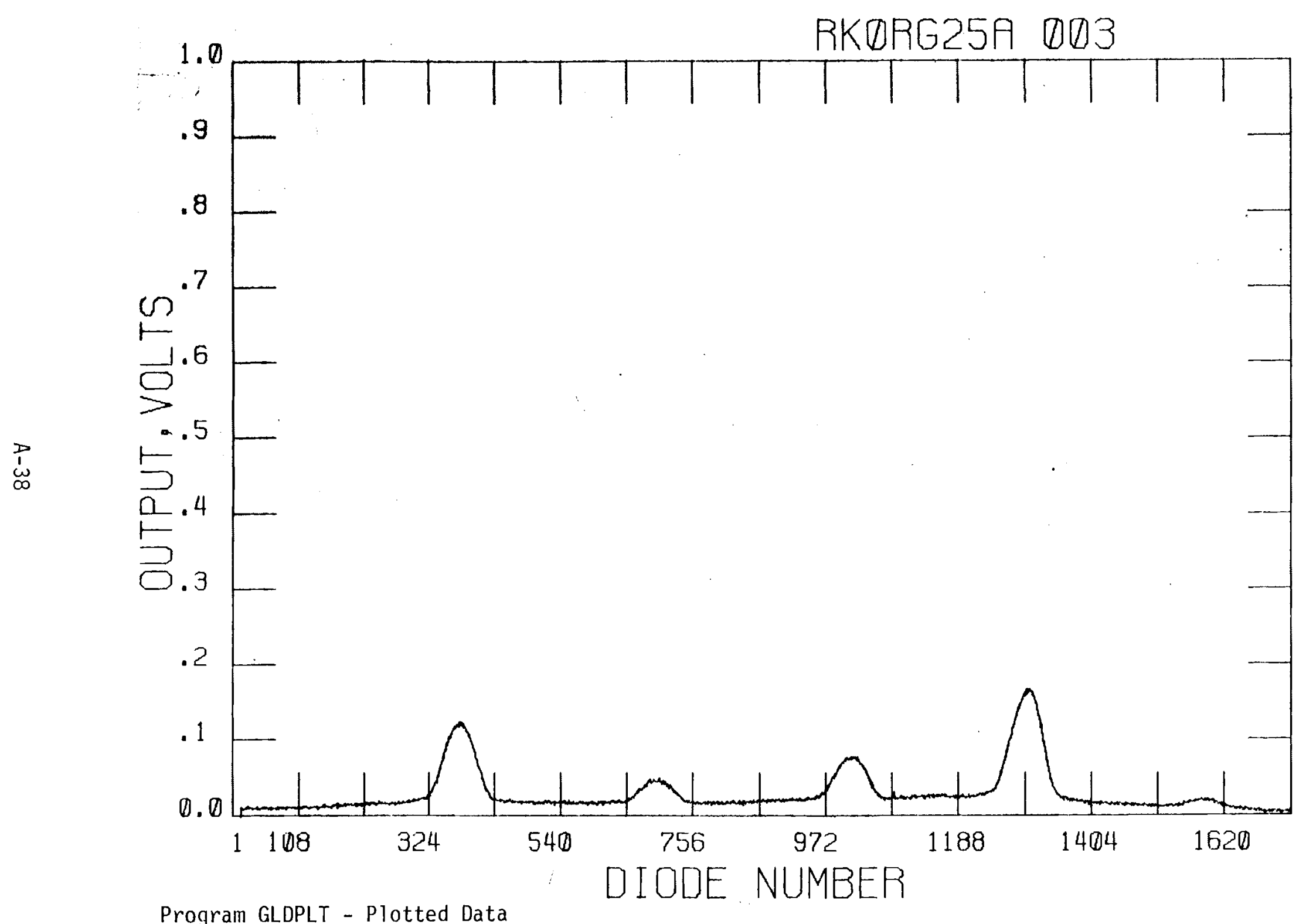




\section{A.4 BIBLIOGRAPHY}

Readers who are interested in the details of the programs discussed in this appendix are referred to the following documents. Definition and use of the software support for the DEC Laboratory Peripheral System can be found in the FORTRAN/RT-11 Extensions Manual, Order No. DEC-11-LRTEA-C-D, Chapter 3. The DEC System Subroutine Library, SYSLIB, is described in detail in Appendix 0 . of the DEC RT-11 System Reference Manual, Order No. DEC-11-ORUGA-C-D, DN1,DN2. These manuals are available from Digital Equipment Corporation, Maynard, Massachusetts.

The Pacific Northwest Laboratory (PNL) Device Independent Graphics subroutines were written by members of PNL Computers and Information Systems Section and are described in internally distributed documents. Information regarding operaional and TX support can be obtained from Jim Thomas. Device independent graphics and BNWLIB were the responsibility of Vern Crow and Larry Gerhardste in. 


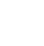
. 
No. of

Copies

OFFSITE

A. A. Churm

DOE Patent Division

9800 S. Cass Avenue

Argonne, IL 60439

W. J. Haubach

DOE Division of Chemical

Sciences

Washington, DC 20545

27 DOE Technical Information Center

ONSITE

DOE Richland Operations Office

H. E. Ransom

27 Pacific Northwest Laboratory

R. L. Gordon (20)

Technical Information (5)

Publishing Coordination (2) 
.

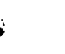
, 